\title{
Cardiac Endothelial-Myocardial Signaling: Its Role in Cardiac Growth, Contractile Performance, and Rhythmicity
}

\author{
DIRK L. BRUTSAERT
}

University of Antwerp, Antwerp, Belgium

I. Introduction
60

II. Endothelial Organization in the Heart 61

A. Cardiac endothelium versus coronary vascular endothelium 61

B. Cardiac endothelial morphology 63

III. Cardiac Endothelial-Myocardial Interaction 65

A. Cardiac growth

B. Cardiac contractile performance $\quad 77$

C. Cardiac rhythmicity 84

IV. Cardiac Endothelial Dysfunction: Role in the Pathogenesis of Cardiac Failure 88

A. Endothelial activation and dysfunction 88

B. Peripheral vascular endothelial dysfunction in cardiac failure 89

C. Coronary endothelial dysfunction in cardiac failure 89

D. Cardiac endothelial dysfunction in cardiac failure 90

$\begin{array}{ll}\text { E. Role of NO } & 91\end{array}$

F. Role of ET

G. Role of other myocardial-endothelial signaling pathways 93

V. The Endothelial System: Conjectural Role of Cardiac Endothelium 94

VI. Summary 98

Brutsaert, Dirk L. Cardiac Endothelial-Myocardial Signaling: Its Role in Cardiac Growth, Contractile Performance, and Rhythmicity. Physiol Rev 83: 59-115, 2003; 10.1152/physrev.00017.2002._Experimental work during the past 15 years has demonstrated that endothelial cells in the heart play an obligatory role in regulating and maintaining cardiac function, in particular, at the endocardium and in the myocardial capillaries where endothelial cells directly interact with adjacent cardiomyocytes. The emerging field of targeted gene manipulation has led to the contention that cardiac endothelial-cardiomyocytal interaction is a prerequisite for normal cardiac development and growth. Some of the molecular mechanisms and cellular signals governing this interaction, such as neuregulin, vascular endothelial growth factor, and angiopoietin, continue to maintain phenotype and survival of cardiomyocytes in the adult heart. Cardiac endothelial cells, like vascular endothelial cells, also express and release a variety of auto- and paracrine agents, such as nitric oxide, endothelin, prostaglandin $\mathrm{I}_{2}$, and angiotensin II, which directly influence cardiac metabolism, growth, contractile performance, and rhythmicity of the adult heart. The synthesis, secretion, and, most importantly, the activities of these endothelium-derived substances in the heart are closely linked, interrelated, and interactive. It may therefore be simplistic to try and define their properties independently from one another. Moreover, in relation specifically to the endocardial endothelium, an active transendothelial physicochemical gradient for various ions, or blood-heart barrier, has been demonstrated. Linkage of this blood-heart barrier to the various other endothelium-mediated signaling pathways or to the putative vascular endothelium-derived hyperpolarizing factors remains to be determined. At the early stages of cardiac failure, all major cardiovascular risk factors may cause cardiac endothelial activation as an adaptive response often followed by cardiac endothelial dysfunction. Because of the interdependency of all endothelial signaling pathways, activation or disturbance of any will necessarily affect the others leading to a disturbance of their normal balance, leading to further progression of cardiac failure. 


\section{INTRODUCTION}

This review is about the interactions beween endothelial cells in the heart and adjacent cardiomyocytes and about how this cross-talk determines cardiac growth, contractile performance, and rhythmicity.

After the discovery in 1980 by Furchgott and Zawadski (186) of the obligatory role of the vascular endothelium in vasomotor tone, the endothelium has emerged as an essential structural and functional element of the cardiovascular system. Subsequent contributions by numerous eminent investigators have over the past 20 years profoundly altered our thinking about the cardiovascular system and redefined many of our working concepts (31, $32,101,114,187,198,204,234,235,396,402,454,465,598$, 600-602, 604, 642). In 1998, Furchgott was awarded the Nobel Prize, together with Louis Ignarro and Ferid Murad, for their contributions to the discovery of the endothelium-derived relaxing factor, nitric oxide (NO) $(396,432)$. Furchgott's theorem about the central, obligatory role of the endothelial cell in cardiovascular regulation has, however, been of much more fundamental and conceptually of far greater importance in biomedical sciences (185).

The endothelium contributes to cardiovascular homeostasis not only by regulating vascular permeability but also by adjusting the caliber of blood vessels to hemodynamic and hormonal demands and by maintaining blood fluidity. Endothelial cells perform these functions by the expression, activation, and release of powerful vasoactive substances as well as of numerous other bioactive molecules. These substances include vasoconstricting and vasodilating factors, pro- and anticoagulant factors, pro- and antithrombotic factors, growth and antigrowth factors, factors that contribute to angiogenesis and tissue remodeling, as well as to immune reactions and tissue inflammation. The endothelium thus creates a complex and finely tuned balance of interactions with the immediate environment. These interactions are common to all endothelial cells. Regional and species differences in the set point of these balances may, however, result in substantial organ- or vascular bed-specific phenotypic diversity of endothelial cell function $(31,198,236,295,313$, $320,355,453,478,484$ ).

In 1986, we proposed that endothelial cells in the heart might similarly play an obligatory role in regulating and maintaining cardiac function. Cardiomyocytes represent the bulk of cardiac tissue mass constituting $\sim 75 \%$ of total tissue volume in the heart, but it has been estimated that their number accounts for $<40 \%$ of all cardiac cells, the majority of cells being highly adaptive nonmyocyte cells, such as fibroblasts, macrophages, circulating blood cells, vascular smooth muscle cells, and endothelial cells. We observed that the contractile performance of isolated cardiac muscle could be profoundly modified by the presence of intact endocardial endothelial cells (55, 62, 63).
Modulation of the contractile state of subjacent cardiomyocytes by endocardial endothelial cells was subsequently extended to include the contribution of the endothelium in the myocardial capillaries $(323,366,414,470)$. Further observations both in vitro and in vivo on aspects of the effects of cardiac endothelium and of related autocrine and paracrine factors and cytokines on cardiac function were made by many investigators in various animal species, including in humans $(24,48,67,81-83,104,146$, 205, 206, 326, 372, 442, 446, 447, 511, 539, 543, 611) (Fig. 1).

As a result, our insights into cardiac function have evolved from an autoregulatory muscular pump, with coronary perfusion and neurohormonal control as the sole important modulators, to a pluricellular, multifunctional organ, in which the endothelial cell plays an essential role with respect to cardiac metabolism, growth, contractile performance, and rhythmicity. Although it is inconceivable to regard the control of blood vessels and the peripheral circulation without considering the obligatory role of the vascular endothelial cell, it is surprising that many continue to approach cardiac function merely in terms of its cardiomyocytes, coronary blood supply, and neurohormonal drive.

The present review focuses on the interactions between the cardiomyocytes and the cardiac endothelial cells of the endocardium and the intramyocardial capillaries, where the endothelial cell is closely apposed to the cardiomyocytes.

The main objectives of this review are 1) to discuss how cardiac endothelial cells interact with their immediately sub- and adjacent cardiomyocytes to control and maintain cardiac growth, contractile performance, and rhythmicity; and 2) to review current knowledge of cardiac endothelial activation and dysfunction and, in particular, how phenotypic changes may contribute to the pathogenesis of cardiac disease leading to cardiac failure.

These objectives are approached from the point of view of a traditional clinical physiologist observing global cardiac function. The emphasis is on the selective integration of all cardiac endothelium-myocardium-mediated signaling pathways within global organ structure and function. Preference has intentionally been given to whole organ integration, while at the same time selectively incorporating some of the molecular biology and genetics of more recently discovered signaling pathways which have emerged from the growing field of targeted gene manipulation.

Focusing merely on cardiac endothelial-myocardial interactions may at first seem to create a rather restrictive view on cardiac function. While avoiding wherever possible the ever-growing number of intracellular signaling pathways omnipresent in all cardiac cells but redundant, i.e., not indispensable, for the strict endothelial-myocardial reciprocal interactions, such apparent "narrow window" approach may, moreover, overlook strictly myocar- 

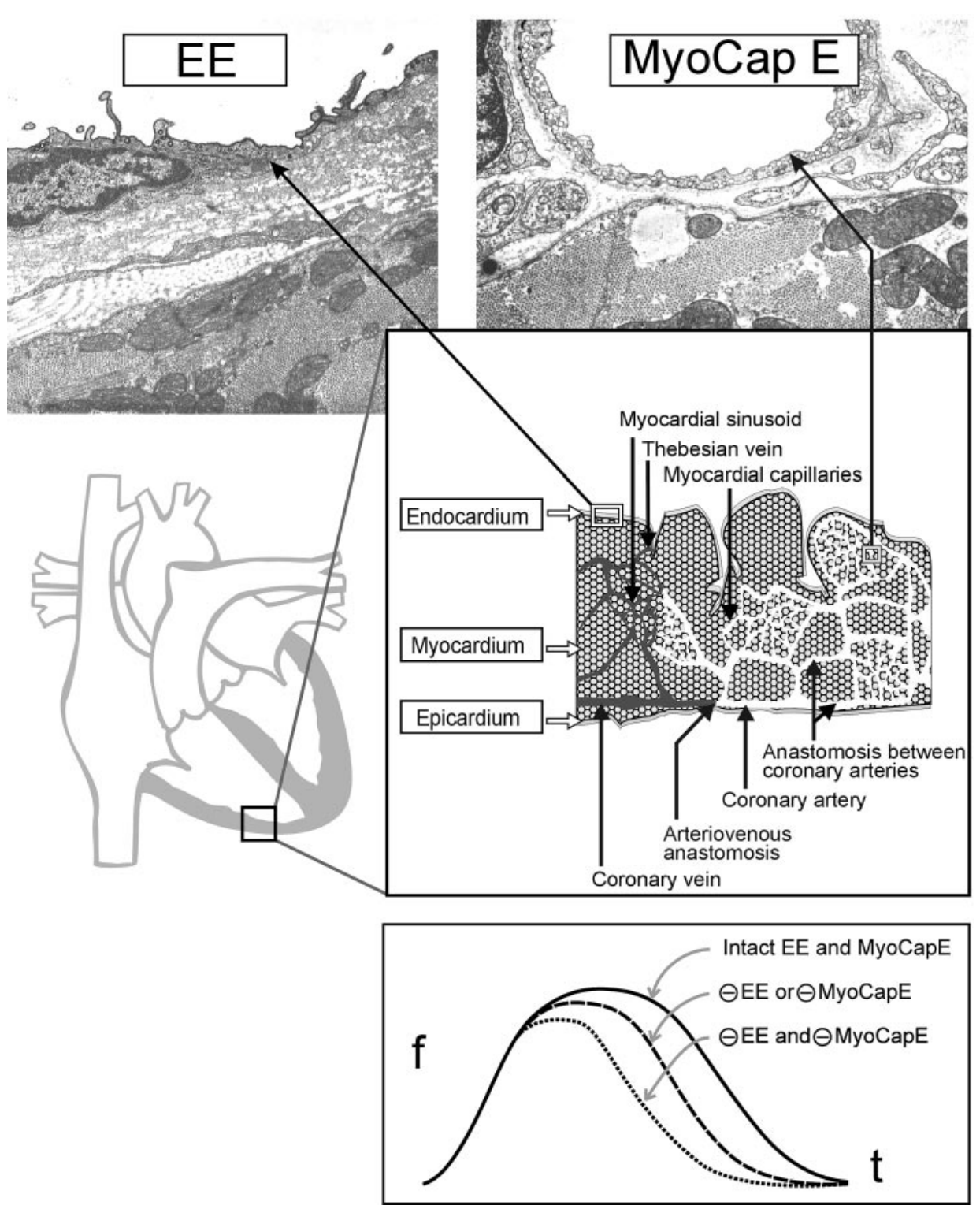

FIG. 1. Cardiac, i.e., endocardial (EE) and myocardial capillary (MyoCapE), endothelium and myocardium. The ventricular wall (inset) consists of the epicardium, the myocardium, and the endocardium. The endocardium includes a fibroelastic layer, a basement membrane, and a luminal layer of endothelial cells, i.e., the EE [top left transmission electron microscopy (TEM) micrograph]. The distance between the MyoCapE (top right TEM micrograph) and cardiomyocytes is usually $<1 \mu \mathrm{m}$; between EE and cardiomyocytes, the distance ranges from $1 \mu \mathrm{m}$ in small mammals to $>50 \mu \mathrm{m}$ in humans. Analogous, and additive, myocardial actions of $\mathrm{EE}$ and MyoCapE result in contractile twitch prolongation and increased peak force development. The traces in the bottom panel represent isometric twitches (force $f$ vs. time $t$ traces) from isolated papillary muscles with intact EE and MyoCapE, compared with twitches from muscles after selective EE damage ( $-\mathrm{EE}$ ) by quick immersion of the papillary muscle in $0.5 \%$ Triton X-100 and to twitches from muscles with MyoCapE dysfunction (-VE) induced by a bolus injection of diluted Triton $\mathrm{X}-100$ in a Langendorff heart before isolation of the papillary muscle. Endothelial damage or dysfunction induced premature relaxation and concomitantly decreased peak force development. [Modified from Brutsaert et al. (60).] dium-related features of the heart (171). It also precludes other cellular interactions which may be equally important or indispensable for the maintenance of the cardiac phenotype, such as between fibroblasts or monocytes/ macrophages or epicardial mesothelial cells, with either cardiomyocytes or with cardiac endothelial cells, among others $(137,209,210,374,617)$. This approach has, however, provided novel insights. Focusing on cardiac endothelial-myocardial interactions has led to an innovative conceptual paradigm of cardiac function which challenges the centripetal, myocardium-oriented conception of cardiac structure and function to which we have become accustomed.

Review articles, published over the past decade (6, $22,23,57-59,263,281,325,442,519,522,625)$, offer more comprehensive coverage of selected aspects and may provide a valuable supplement to this review.

\section{ENDOTHELIAL ORGANIZATION IN THE HEART}

\section{A. Cardiac Endothelium Versus Coronary Vascular Endothelium}

In the postnatal and adult heart, endothelial cells can affect cardiac function in different ways. It is indeed important to distinguish between the contribution of the cardiac endothelial cells in the myocardial capillaries and at the endocardium and the contribution of the coronary vascular endothelium in the major epicardial and smaller intramyocardial coronary arteries and veins (Fig. 2). The vascular endothelium in the coronary conduit and resistance vessels controls coronary artery function as in any other vascular bed in the body, but its contribution to 


\section{Vascular Endothelium}
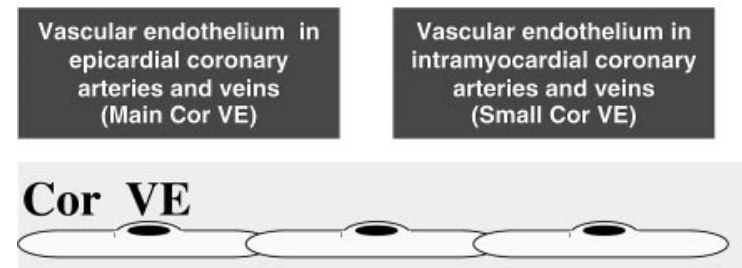

SMOOTH
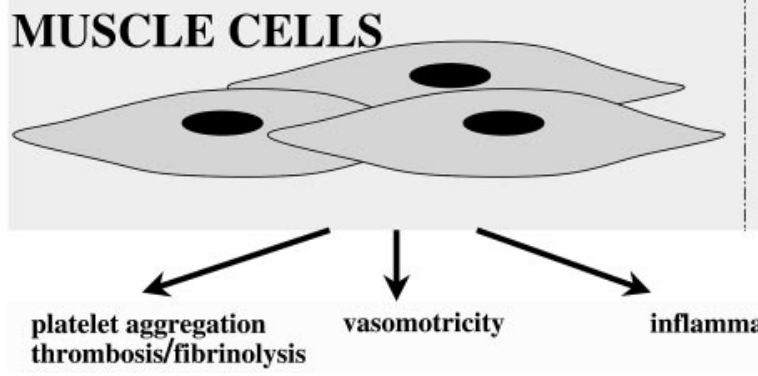

\section{Cardiac Endothelium}
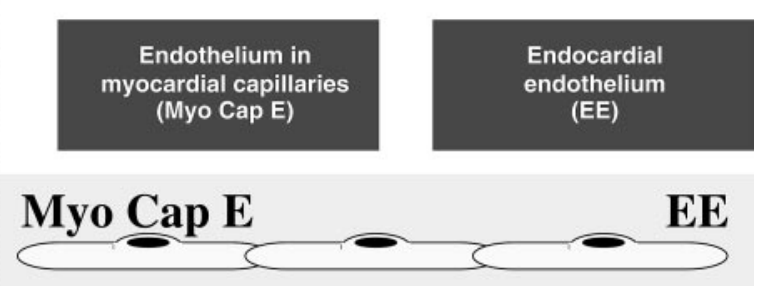

CARDIOMYOCYTES

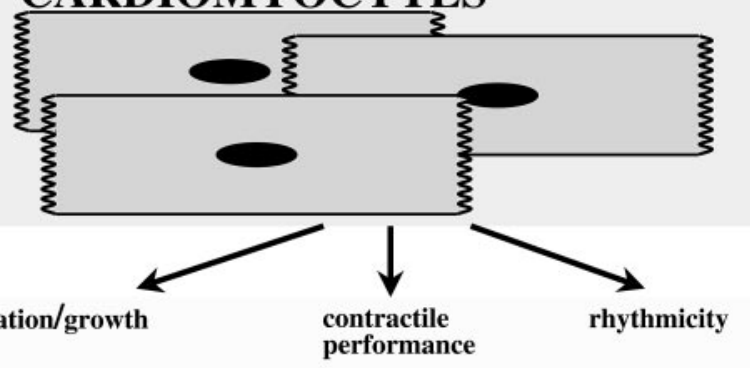

FIG. 2. Organization of coronary vascular (CorVE) and cardiac (MyoCapE, EE) endothelial cells in the heart. Right: a direct signaling exists between the MyoCapE or the EE and the immediate subjacent cardiomyocytes with effects on cardiac growth, contractile performance, and rhythmicity. Left: the epicardial and intramyocardial CorVE affect the myocardium only indirectly through changes in coronary vasomotricity, hence through myocardial blood supply.

cardiac function is indirect by controlling coronary blood supply to the myocardium. Cardiac endothelial cells in the myocardial capillaries (MyoCapE) and in the endocardial endothelium (EE), in contrast, are in close proximity to adjacent cardiomyocytes, allowing for direct cellular communication and signaling between both cell types.

Crucially important for endothelial cell-to-cardiomyocyte interactions at these two sites is their intercellular distance and their cell number ratio. The latter depends on the capillary-to-cardiomyocyte ratio and intercapillary distance, which will vary with species and cardiac sampling site. In left ventricular wall and papillary muscle of adult rat $(13,469,591)$ and neonatal mice $(72)$, capillary-to-cardiomyocyte number ratio may on crosssectional view vary from 0.91 to 1.12 . The lower figure of 0.5 observed in human "endomyocardial" biopsies from right ventricular trabeculae (401) can be ascribed to the close proximity of endocardial endothelial cells that are the dominant endothelial cells in this zone. In fact, cardiac endothelial cells outnumber cardiomyocytes by $3: 1$, although cardiac endothelial cell-to-cardiomyocytal volume (or mass) ratio is of the order of only 0.04-0.05 (13). The intercapillary distance was reported to be $20.2 \mu \mathrm{m}$ in the ventricular wall (591) and $15.6 \mu \mathrm{m}$ in papillary muscle (13) of normal rat heart and $6 \pm 0.4 \mu \mathrm{m}$ in normal neonatal mice heart (72). With a distance of $\sim 1 \mu \mathrm{m}$ between the capillary endothelial cell and the nearest cardiomyocyte, this provides for an action (diffusion) radius of $\sim 3-12 \mu \mathrm{m}$ for each capillary endothelial cell into the neighboring cardiomyocytes $(72,468,469,583)$. This would suffice for an efficient endothelial-myocardial signaling agent, even for those with short half-lives, such as endothelium-derived nitric oxide (NO) with its biological half-life of $20 \mathrm{~s}$ and whose liposolubility will further aid its diffusion. NO diffuses from its source over a range of 150-300 $\mu \mathrm{m}$ within 4-15 s (303); diffusion distance may even reach $600 \mu \mathrm{m}$ (276).

The closest distance from endocardial endothelial cells to subjacent cardiomyocytes, depending on animal species and cardiac site, varies from $<1 \mu \mathrm{m}$ in small mammals to $10-30 \mu \mathrm{m}$ in the ventricle and $>50 \mu \mathrm{m}$ in atria of larger mammals, including humans. In the subendocardial space, additional interactions of the endocardial endothelial cells with the subendocardial terminal Purkinje fiber network and with the extensive subendocardial neural plexus $(95,96,330$, $359,644)$ may be equally important.

It appeared from our original experimental observations that the actions of EE and MyoCapE on myocardial contractile performance were additive, and analogous if not identical. Although EE and MyoCapE share common features in their effects on subjacent cardiomyocytes, these two cardiac endothelial cell types are, however, not identical. They differ with regard to developmental, morphological, and functional properties, the major difference probably resulting from the way in which they perceive and transmit signals and from their different hierarchic position and contribution within the overall endothelial system. 


\section{B. Cardiac Endothelial Morphology}

In vertebrates, the luminal surface of the mature heart is structurally complex, comprising furrows, cylinder- and sheetlike trabeculae, and papillary muscles. In the human heart, the architecture of the ventricular wall is more elaborate in the right than in the left ventricle. In the left ventricle, trabeculations are more densely organized at the apex and posterior wall than on the septum whose surface is usually smooth; in the right ventricle, intense trabeculation may constitute over $50 \%$ of wall thickness. The complex cavitary surface of the cardiac wall is completely lined by the EE. The morphology of the $\mathrm{EE}$ has been well described (6). It is recognizable as a sheet of endothelial cells with a central nuclear bulge and distinct, extensive intercellular junctions. EE cells are somewhat larger than endothelial cells in most other portions of the circulatory system. The luminal surface of most of these cells possesses a scattered variety of microappendages, or microvilli, projecting into the cardiac cavity. It can be estimated that the labyrinth of trabeculae and furrows, together with the numerous microvilli on the luminal surface of the EE cells, may augment the surface area by a factor of 100 or more. This surprisingly large contact surface area of the EE offers an astonishingly high ratio of cavitary surface area to ventricular volume, particularly in the right ventricle. This suggests an important sensor role for the EE (55).

The intercellular clefts between EE cells are three to five times deeper than in MyoCapE (Fig. 3) and are often highly tortuous with two or three cells imbricated. Most clefts contain one or two tight junctions. Only in a few areas, depending on age and on species, are EE cells separated by simpler clefts that are shallower and lacking tight junctions (compare Fig. 3, top and bottom left). In MyoCapE, in contrast, most clefts are simple, shallow, and exhibit few tight junctions with many interruptions $(66,614)$. The degree of cellular overlap in the EE thus exceeds that in MyoCapE three- to fivefold as confirmed by experiments in which cardiac endothelial cells were stained with an antibody recognizing an endothelial marker (platelet-endothelial cell adhesion molecule; PECAM) (Fig. 4, top).

PECAM belongs to the immunoglobulin superfamily and participates in the establishment and maintenance of barrier function and permeability in many endothelia (105a, 162, 211).

In the EE, PECAM-1 staining is typically confined to the border zone of the EE cells corresponding to the zone of cellular overlap and intercellular clefts (8). In MyoCapE, in contrast, PECAM-1 stained more diffusely over the entire cell surface. The pronounced difference in distribution of PECAM-1 suggests profound differences in transendothelial permeability between these two cardiac sites. Transendothelial transport is believed to be mediated by diffusion through the intercellular clefts, or by nondiffusional vesicular transport, or through cell-matrix junctions, i.e., focal adhesion contacts (340). The paucity in most cavitary EE cells of central actin stress fibers, which normally limit the adhesion of endothelial cells to

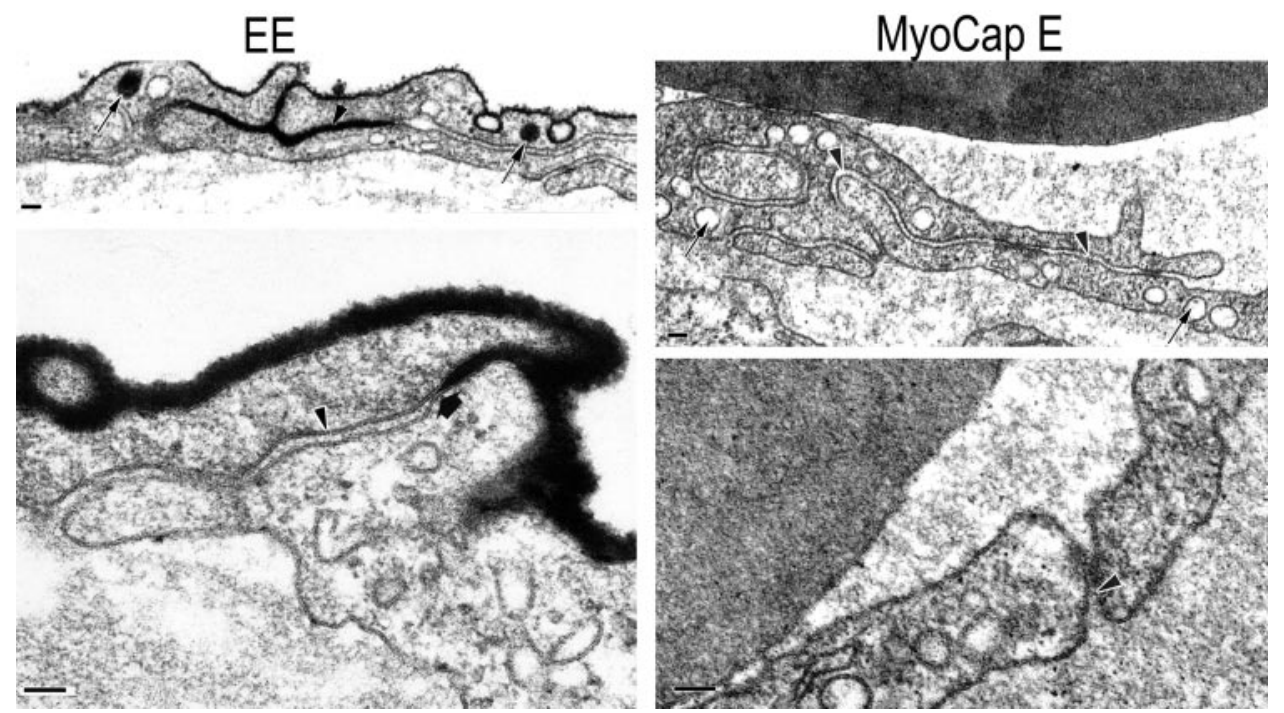

FIG. 3. Transmission electron micrographs of junctional areas of cardiac endothelium from rat left ventricle. Left: junctional areas in endocardial endothelium (EE) show extensive cellular overlap. The tortuousness of the majority of intercellular clefts (upper) is in contrast to the rarely observed more simple, straight intercellular cleft (lower). The cell coat of the apical surfaces, including that of luminal vesicles and of apparently cytoplasmic vesicles (arrows in top panel), and part of the deep intercellular clefts (arrowheads) are stained with tannic acid. Penetration of tannic acid was interrupted at the first punctate junctional contact (thick arrow in bottom panel). Right: junctional areas in endothelium of myocardial capillaries (MyoCapE) show little cellular overlap. A typical intercellular cleft (arrowhead in bottom panel) is simple, shallow, and displays few, often interrupted tight junctions. The most complex intercellular clefts in MyoCapE, as, e.g., in top right, are comparable to the least complex clefts in EE, as in bottom left. Scale bars, $50 \mathrm{~nm}$. [Modified from Brutsaert et al. (60) and kindly provided by Luc Andries.] 

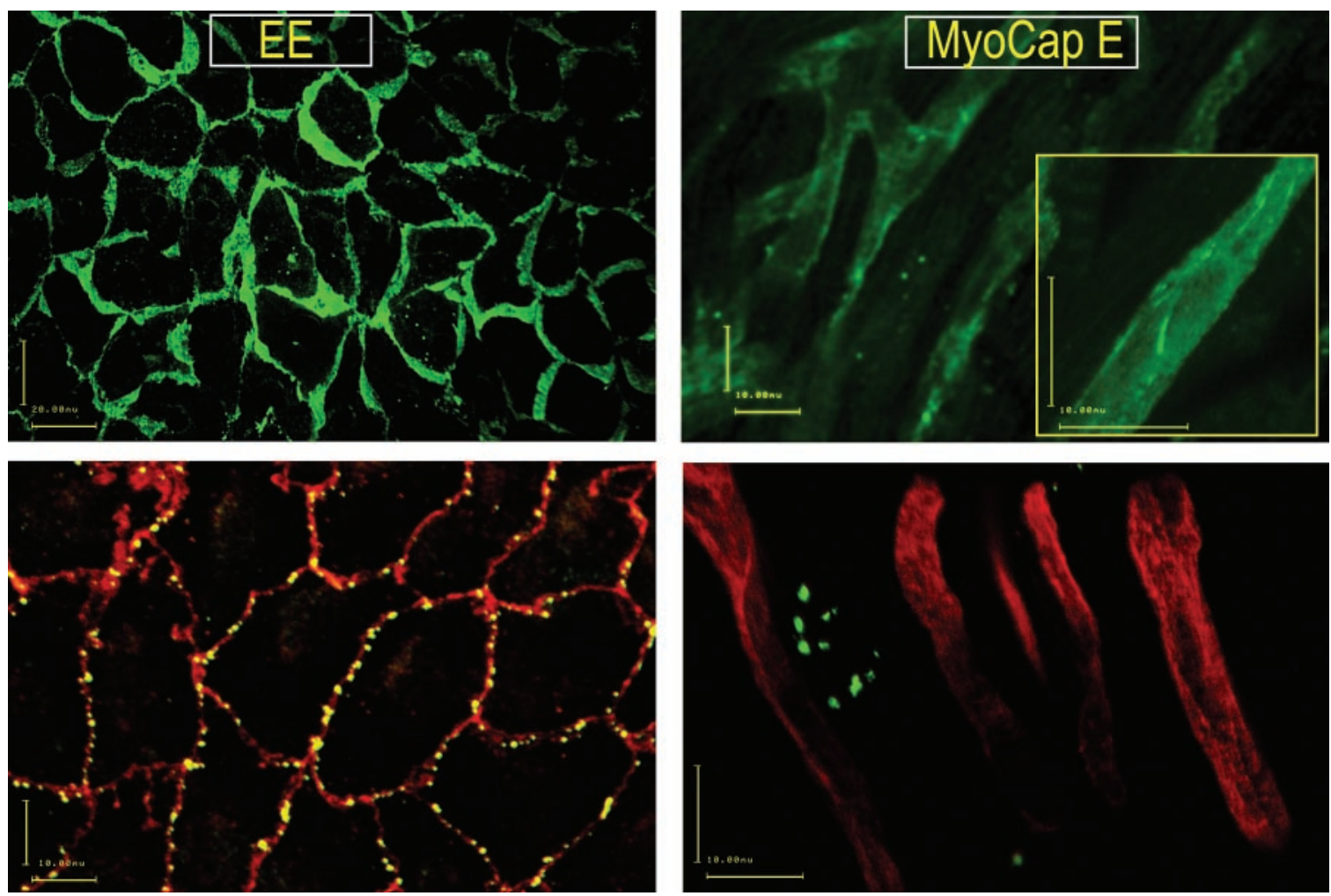

FIG. 4. Confocal scanning laser micrographic optical sections of junctional areas and gap junctions in cardiac endothelium. Top: platelet endothelial cell adhesion molecule (PECAM)-1 staining of optical sections through the endocardial endothelium (EE) and through the myocardium of the rabbit left ventricle. The width of the stained borderzones of EE cells (left) reflects the depth of the intercellular clefts from the luminal to the abluminal opening. In myocardium (right), PECAM-1 staining is confined to the endothelium of myocardial capillaries (MyoCapE) and is distributed more diffusely over the entire endothelial cell surface. The stronger stained linear marks $($ inset ) might reflect either intercellular junctions between MyoCapE cells or membranous folds. Notice the different scale bars in left (20.00 $\mu \mathrm{m})$ and right $(10.00 \mu \mathrm{m})$. Bottom: dual connexin43 (Cx43) and PECAM-1 (left) or rat endothelial cell antibody (RECA) (right) immunostaining of optical sections through EE of the right ventricular outflow tract (left) and through the myocardium of the right ventricle (right) of the rat were obtained by merging two separate images. The green color represents Cx43; the red color represents PECAM-1 in the left panel or RECA in the right panel; where both Cx43 and PECAM-1 or RECA were present, the color is fused to yellow. In EE (left), Cx43-stained gap junctions had variable sizes and outlined the PECAM-1-stained cell borders; nuclei of EE cells could be discerned as dark spots. In the myocardium (right), RECA staining delineated the MyoCapE cell membranes, but Cx43-stained gap junctions were restricted to the myocytes. [Modified from Brutsaert et al. (60) and kindly provided by Luc Andries.]

substrate matrix proteins, suggests that transport through focal adhesion contacts is probably of less importance in EE. The abundance of vesicles in MyoCapE compared with the scarcity of vesicles in EE similarly suggests that vesicular transport would be less prominent in EE than in MyoCapE. It would thus appear that trans-EE-permeability is controlled predominantly through the intercellular clefts, as mediated through 1) the extent and complex structure of the clefts which are, moreover, lined by a dense electrically charged glycocalyx; 2) the presence of one or two tight junctions (zonula occludens); and 3) the presence of a well-organized zonula adherens with complex interactions between a circumferential actin filament band and several connecting proteins, e.g., vinculin. Costaining of EE for actin and for nonmuscle myosin (6) has suggested that changes in trans-EE-permeability could be mediated not only by stretch (431) or by passive retraction of the EE cells, e.g., by phosphorylation of actin-linking proteins, such as vinculin and $\alpha$-catenin, but also by activation of actin-myosin interactions.

EE and MyoCapE differ also in the way by which they communicate with adjacent endothelial as well as subjacent nonendothelial cells (59). The abundance of gap junctions between EE cells (Fig. 4, bottom left), as evident from transmission electron microscopy and immunostaining with several connexins (Cx43, Cx40, Cx37), and their absence in MyoCapE (Fig. 4, bottom right) suggest that EE displays an intimate electrochemical linkage among neighboring EE cells. This type of communication would allow for rapid intercellular electrochemical spreading of the functional properties of EE after activation of even a single EE cell (39). The ensuing amplification, moreover, would be in accordance with their suggested sensor function. The lack of gap junctions in MyoCapE, in contrast, 
suggests a more local control of myocardial function. Major differences were also observed in relation to the potential for communicating with nonendothelial cell types. Intercellular adhesion molecule-1 (ICAM-1) and antigens of the major histocompatibility complex (MCHI, MCHII and, DR) were more prominent in MyoCapE than in EE, PECAM was differently distributed, and the endothelial markers PAL-E and von Willebrand's factor were more abundant in EE than in MyoCapE (7).

\section{CARDIAC ENDOTHELIAL-MYOCARDIAL INTERACTION}

EE and MyoCapE share nevertheless many common features, typical for cardiac endothelial cells, and likely to be critical for normal cardiac growth, contractile performance, and rhythmicity. They reflect direct endothelialcardiomyocytal interactions, e.g., through reciprocal signaling by peptide growth factors, through auto- and paracrine mechanisms, and, at least for the endocardial endothelium, through an active transendothelial bloodheart barrier. As noted above, the involvement of $\mathrm{EE}$ and MyoCapE in cardiac growth, contractile performance, and rhythmicity must be clearly distinguished from the functional role of coronary vascular endothelial cells in the heart. The latter endothelial cells act only indirectly through changes in coronary vasomotor tone, hence in myocardial blood supply. Because coronary vascular endothelial cells fall outside the scope of the present review, they are not considered further. The distinct features of $\mathrm{EE}$ and MyoCapE on cardiac growth, contractile performance, and rhythmicity will now be dealt with in greater detail (Fig. 5).

\section{A. Cardiac Growth}

The modulating role of the vascular endothelial cell on vascular, including coronary, growth is widely recog-

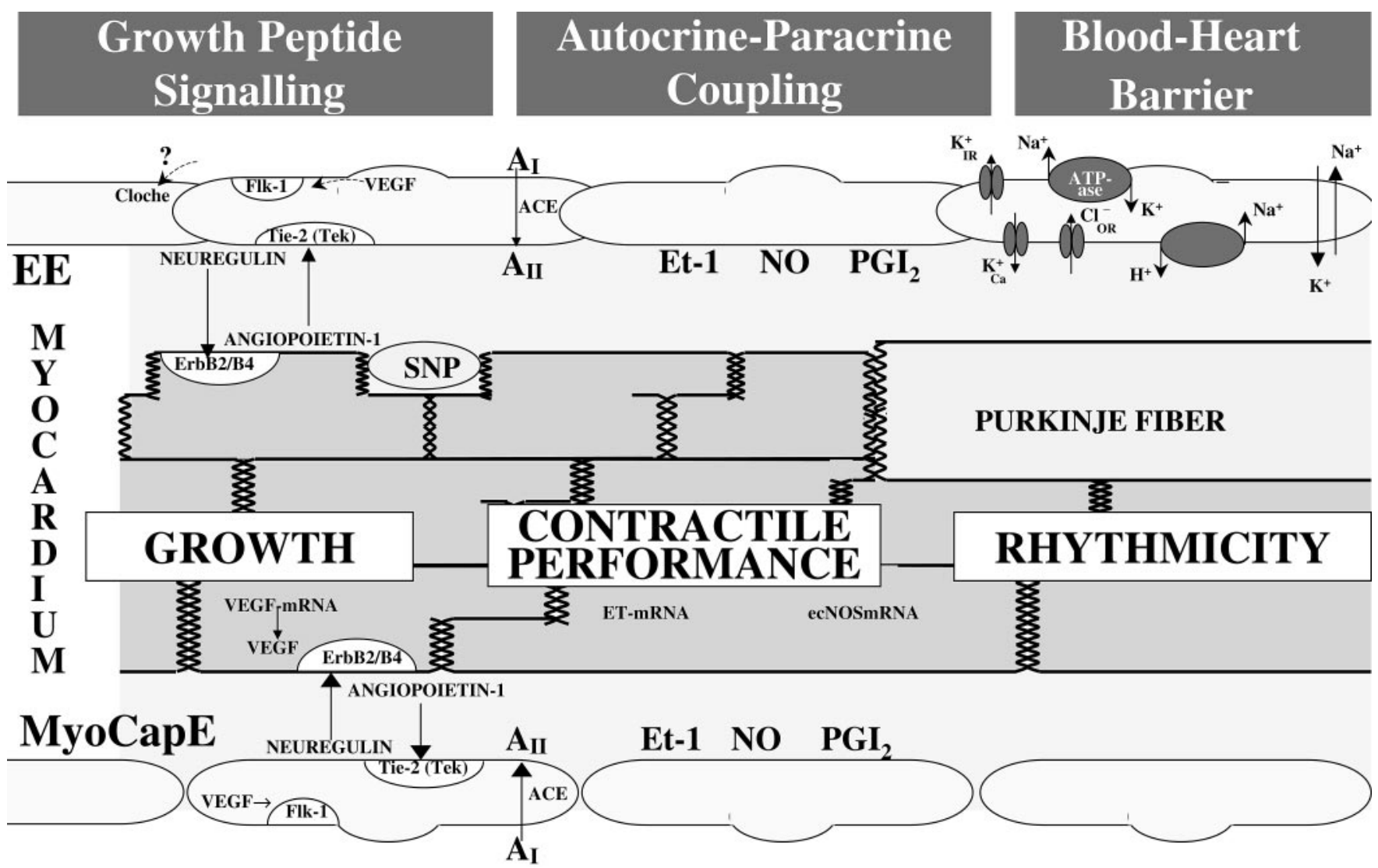

FIG. 5. Reciprocal signaling between cardiac endothelial cells and cardiomyocytes influences myocardial growth, contractile performance, and rhythmicity. MyoCapE interacts with cardiomyocytes through autocrine-paracrine coupling and growth peptide signaling. The EE may, in addition, act through an active blood-heart barrier mechanism. EE cell-to-cell coupling through gap junction allows for rapid electrochemical intercellular spreading and amplification of functional properties. Tight junctions and zonula adherens participate in the control of trans-EE-permeability through overlapping intercellular clefts. The Purkinje fiber network and the subendocardial neural plexus (SNP) run immediately subjacent to the EE and may further endorse endothelial control of cardiac rhythmicity. Et-1, endothelin-1; NO, nitric oxide; $\mathrm{PGI}_{2}$, prostacyclin; $\mathrm{AI}$ and AII, angiotensins I and II; VEGF, vascular endothelial growth factor. 
nized. The development of a vascular supply through either vasculogenesis (in situ formation of vessels) or angiogenesis (vessel formation through outgrowth or branching) is a fundamental requirement for embryonic organ development as for wound healing and tissue regeneration in adult life. The search for potential angiogenic growth factors or regulators has yielded numerous candidates. A pivotal role in the regulation of normal and abnormal vascularization of all, including coronary vessels, has been ascribed to the specific vascular endothelial growth factor (VEGF) and the fibroblast growth factor (FGF) $(439,581,582)$ among other more recently discovered molecules. We would refer the reader to the considerable literature on the subject $(70-72,145,158-160,167$, 214, 529).

Much less is known about the impact on myocardial growth by cardiac endothelial cells (MyoCapE and EE). Whether and to what extent cardiac endothelial cells control cardiogenesis in the embryo or play a role in cardiac remodeling in the adult is under intensive investigation. Much work has been done on growth-modulating properties of the various auto- and paracrine factors, such as NO, prostacyclin, endothelin, and angiotensin, released or activated by cardiac endothelium and their possible role in hypertrophic responses or "remodeling" in the adult heart. Most of our present knowledge about the obligatory link between cardiac endothelial cells and cardiomyocytes with respect to growth comes from the rapidly expanding field of developmental cardiology, particularly from experiments on embryonic development in transgenic animal models $(19,164,558)$.

\section{Cardiac development}

In the vertebrate embryo, the heart develops from the cardiogenic mesoderm to form the double-walled primary heart tube. This tube consists of only two cell types, i.e., endocardial endothelial cells of the inner layer, which is separated by an extracellular, amorph elastic matrix, the cardiac jelly, from the outer layer which consists of a mesh of cardiomyocytes. Endocardial endothelial cells and cardiomyocytes appear at about the same time during development. Based on morphological heterogeneity within the cardiogenic mesoderm, the two cell lineages were at first thought to develop rather independently from one another. There still remains some controversy, however, as to whether myocardial and endocardial lineages have a common or a separate origin $(140,339,381,382$, 384 ). In other words, although cardiomyogenic and cardiac endothelial progenitors are both derived from lateral plate mesoderm, the relationship of these cell lineages in the embryo is unresolved. It is clear, however, that these two cell lineages diversify before the primary heart is formed. The cardiomyocytes express muscle-specific genes and proteins long before initiation of heart beat
(215, 647). Endocardial endothelial cells first express an endothelial marker (the QH-1 antigen) as they segregate from the epithelialized cardiogenic mesoderm (329, 560). In an immortalized cardiogenic mesodermal cell line, the QCE-6 cell, both cardiac myogenic and endothelial cell lineages could be commonly induced in response to several different growth factors (139). Retroviral cell lineage studies do not however support a common origin from the cardiogenic mesoderm, but show rather that the cardiogenic mesoderm consists of two distinct subpopulations (88). Similarly, studies in zebrafish (309) identified that the two lineages have already separated from a common progenitor cell at the blastula stage, before formation of mesoderm. Some of these studies (139) have indicated that appropriate levels of retinoic acid, the major active metabolite of vitamin A, are required for the initial formation of myocardial and endocardial lineages and of the primary heart tube. Quail embryos cultured in the absence of retinoic acid have an underdeveloped endocardium (223) and fail to form the primary heart tube (285).

Endocardial endothelial cells subsequently invade the cardiac jelly and migrate toward the myocardial tube. ${ }^{1}$ Soon, the endocardial endothelium comes to line most of the cardiomyocytes as the sole cardiac endothelial cell monolayer. Endocardial endothelium and cardiomyocytes together constitute the primitive spongy heart tube. The first rhythmic cardiac contractions are initiated at this double-walled stage of heart development (352). Some beating myocytes seem to migrate toward the endocardium, generating a number of protrusions or trabeculae (352). The formation of this spongy and trabeculated pattern substantially increases the endocardial endothelial surface area. At a later stage, cardiac valves and septum develop through endocardial cushion formation. Cushion formation is followed by the development of more compact myocardium in the periphery of the developing cardiac walls. The formation of compact myocardium, or myocardial "compaction," is accompanied by the penetration of small buds of primitive coronary vessels invading it from the epicardial surface.

Accordingly, maturation of the heart into a fourchambered muscular pump organ requires at least three essential, consecutive developmental steps: 1) formation of a trabecular myocardial layer, 2) endocardial cushion formation with valve development and septation, and 3)

\footnotetext{
${ }^{1}$ The composition and function of the cardiac jelly as the sole extracellular matrix between both cellular layers are still not well understood. Its role in early cardiogenesis seems though to be more than that of a passive coupling matrix or mechanical buffer between both cellular layers (407, 409). For example, in mutant mice embryos deficient in one of the cardiac jelly's constitutive components, fibronectin, the organization of the myocardial layer is disrupted and the endocardial layer appears collapsed. Early differentiation of cardiomyocytes and endocardial cells proceeds normally, but their subsequent organization is disturbed, leading to embryonic death of the animal (191).
} 


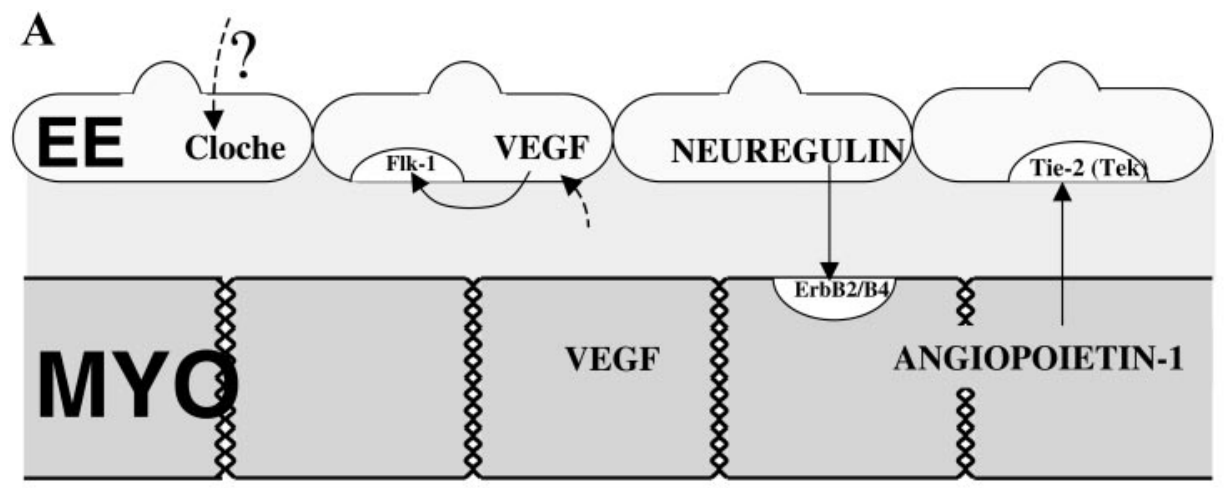

B

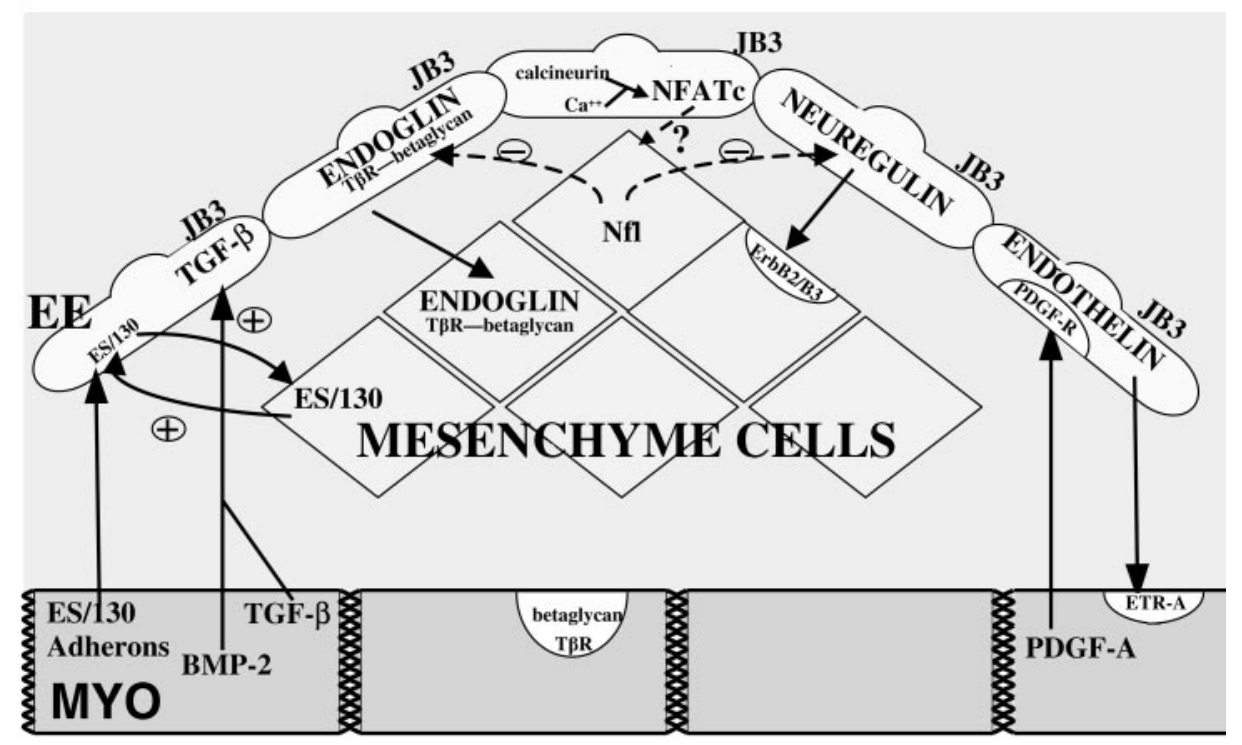

FIG. 6. Obligatory role of endocardialmyocardial signaling in cardiac development, growth, and maturation during early embryonic cardiac differentiation, myocardial trabeculation $(A)$, and endocardial cushion formation $(B)$.

growth and thickening of an outer compact ventricular chamber wall with formation of a coronary circulation (514). Unique and reciprocal signaling pathways between the primitive endocardial endothelial cells and the cardiomyocytes seem to be involved in all these processes.

A) MYOCARDIAL TRABECULATION (FIG. 6, TOP). The spongy, trabecular myocardium is largely responsible for the maintenance of blood flow during early stages of cardiac morphogenesis before expansion of the compact zone. Trabeculation of the spongy heart constitutes the first step in cardiac maturation for which endocardial-myocardial signaling is prerequisite. This signaling is essential for normal maturation and functioning of the heart and for survival.

I) Cloche gene. In cloche mutant zebrafish embryo lacking the inner endocardial endothelial tube, the developing outer myocardial layer remained somewhat smaller and dysmorphic and failed to develop trabeculation within the ventricle $(327,549)$.

Although the single myocardial tube was capable of initiating spontaneous contractions, contractility was markedly reduced with distended atria and collapsed ven- tricles, and survival was brief. The cloche gene is probably the earliest gene presently known to function in endocardial endothelial cell development.

II) VEGF. VEGF and its receptors constitute another indispensable signaling pathway, necessary for the initiation and establishment of mature endocardial endothelialmyocardial interactions. As in the cloche mutant zebrafish embryo, mutation of Flk-1 receptor (VEGFR-2) in mouse also resulted in absence of endocardial endothelium, with subsequent failure of myocardial maturation and embryonic death of the animal $(327,529) .^{2}$ Flk-1 receptor belongs to the family of tyrosine kinase receptors. It is expressed at an early stage in all endothelial cells, including the endocardial endothelium, although later than the cloche gene. In the developing heart, the ligand for Flk-1 receptor, VEGF, is expressed by the trabecular cardiomyocytes as its major source $(387,561)$. Binding with VEGF is required for initial differentiation and proliferation of endocardial endothelium. In contrast, in mice deficient in

\footnotetext{
${ }^{2}$ Flk, fetal liver kinase.
} 
Flt-1 receptor (VEGFR-1), another tyrosine kinase receptor for VEGF, endocardial endothelial cells did differentiate normally, but were disorganized and abnormally thickened, with extra endothelial cells filling up the ventricular cavity, leading to early death of the embryos (167). ${ }^{3}$ These early embryonic lethalities initially inhibited investigating into the role of endocardial-myocardial VEGF signaling at later developmental stages. With the advent of the technique of conditional inactivation, it has become possible specifically to inactivate VEGF-A in a cellular and organ-specific manner during development, prolonging survival. In one such conditional VEGF-A -/mutant mouse, the collagen2a1-Cre VEGF Flox/+, the endocardium appeared detached from the underlying myocardium, which was much thinner with less-developed trabeculae and embryonic lethality (214).

These observations emphasized that, although cardiomyocytes in the early embryo may differentiate normally in the absence of an endocardial tube, endocardial endothelial cells are from the onset of cardiac development prerequisite for myocardial maturation, normal function, and survival. In addition, threshold levels of VEGF-A expression are required for proper endocardial-myocardial interactions.

III) Neuregulin. Further evidence for the obligatory role of the endocardial endothelium in orchestrating myocardial cell maturation and function has been confirmed after the discovery of the neuregulin growth factor signaling pathway in the endocardial endothelium (189, 287, $308,356,380,651) .{ }^{4}$ At an early embryological stage (though later still than cloche and Flk-1 expression), neuregulin- 1 expression is confined to the endocardial endothelium from where it is released as a paracrine growth signal to the tyrosine kinase $\mathrm{ErbB}_{2}$ (HER-2, or Neu) and $\mathrm{ErbB}_{4}$ (HER-4) receptors, expressed on nearby cardiomyocytes. Activation of the $\mathrm{ErbB}_{2} / \mathrm{ErbB}_{4}$ receptor complex by neuregulin is required for trabeculation of the primitive spongy heart. Mutant embryos lacking either one of these three genes exhibit no trabeculation and die in utero at an early stage (Fig. 7).

IV) Serotonin. Serotonin $\left(5-\mathrm{HT}_{2 \mathrm{~B}}\right)$ receptor knockout mice exhibited a significant reduction specifically in ErbB $_{2}$ mRNA with no alteration of $\mathrm{ErbB}_{4}$ mRNA expression (411). The most severely affected $5 \mathrm{HT}_{2 \mathrm{~B}}-/-$ mutant embryos lacked myocardial trabeculation as did mutant $\mathrm{ErbB}_{2}-/-$ embryos. Consistent with previous observations that selective $5-\mathrm{HT}_{2 \mathrm{~B}}$ receptor antagonists led to defects of trabeculation (411), the findings with $\mathrm{ErbB}_{2}$ $-/-$ animals suggest interaction between these two sig-

\footnotetext{
${ }^{3}$ Flt, fms-like tyrosine kinase.

${ }^{4}$ Neuregulin-1 (NRG1) is also called Neu differentiating factor, or heregulin, or glial growth factor (GGF), or acetylcholine receptor-inducing activity.
}

naling pathways already in early heart development (411). 5-HT may indeed act as a cardiac mitogenic factor via a $5 \mathrm{HT}_{2 \mathrm{~B}}$ receptor-mediated pathway, involving the neuregulin-ErbB ${ }_{2}$ signaling pathway. The surviving $5 \mathrm{HT}_{2 \mathrm{~B}}$-deficient mice exhibit severe cardiomyopathy with a loss of ventricular mass due to a reduction in number and size of cardiomyocytes (412).

V) Angiopoietin. An interesting parallel development was the discovery of another family of receptor tyrosine kinases termed TIE. ${ }^{5}$ These receptors are rather specifically expressed in endothelial cells, including embryonic endocardial endothelial cells. Their ligand, angiopoietin-1, which is the first member of a new growth factor-like family specific for the TIE-2 (previously called TEK) receptor, is highly expressed in myocardium surrounding the TIE2-expressing endocardium. ${ }^{6}$ In mutant mice lacking either myocardial angiopoietin-1 or its receptor TIE-2 in the endocardium, a less complex and less well differentiated endocardial endothelial lining was observed; even though the endothelial cells were present in normal number, the endocardial monolayer appeared somewhat collapsed and retracted from the myocardium (461, 500, 562 ). This abnormality too resulted in markedly diminished myocardial trabeculation. Angiopoietin-1 produced by the myocardium thus appears to influence the development of the adjacent endocardium which, in turn, provides key signals required for normal myocardial trabeculation. Angiopoietin-2, in contrast to the myocardium-derived angiopoietin-1, is highly expressed in microvascular endothelial cells $(353,422)$. It is a naturally occurring antagonist of angiopoietin-1 in that it competes for binding to TIE-2 and blocks angiopoietin-1-induced TIE-2 autophosphorylation. Overexpression of angiopoietin-2 in microvascular endothelial cells in transgenic mice resulted in embryonic defects reminiscent of those observed in angiopoietin-1 and TIE-2 knock-out mice (347). The implications with respect to endothelial-myocardial signaling in the developing heart need further investigation.

An essential, direct or indirect transcriptional regulator of angiopoietin-1 and VEGF in the developing heart is the MEF2C gene that is expressed in cardiac muscle cells as well as in endothelial cells. Targeted deletion of MEF2C in mice resulted in dramatically reduced cardiac expression of angiopoietin-1 and VEGF with malformed and irregularly oriented endocardial endothelial cells and severely constricted endocardial lumen (40), loss of the right ventricle, and failure of the remaining ventricle to loop (328). Some of these malformations resemble endo-

\footnotetext{
${ }^{5}$ TIE, tyrosine kinase with immunoglobulin-like loops and epidermal growth factor homology domains.

${ }^{6}$ TEK (TIE-2), tunica interna endothelial cell kinase.
} 


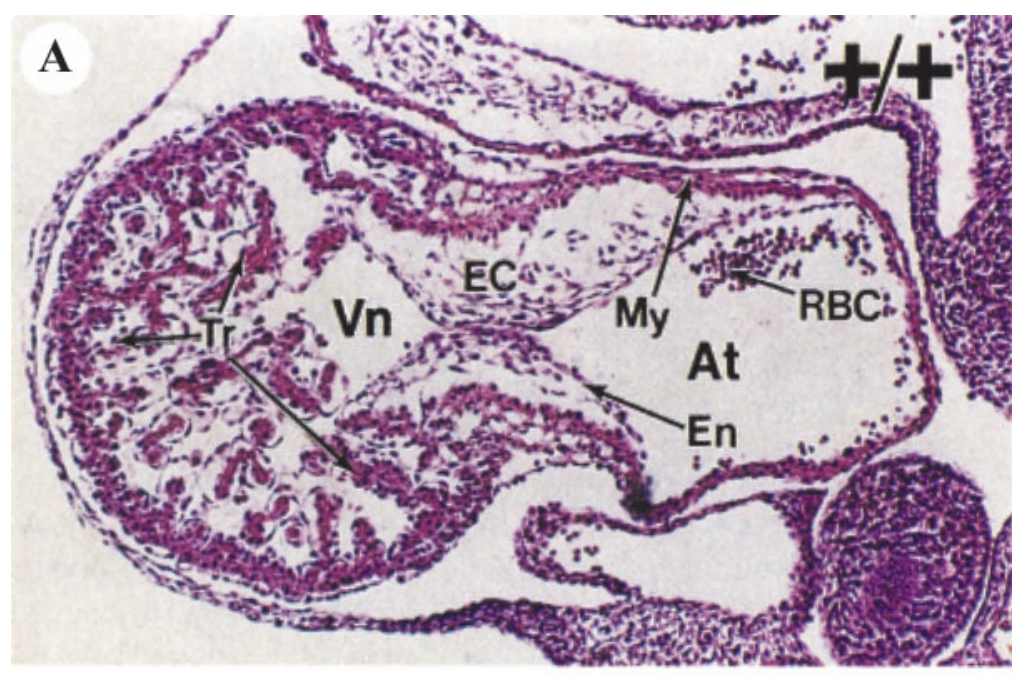

FIG. 7. The neuregulin-ErbB ${ }_{2} / \mathrm{B}_{4}$ pathway is essential for normal trabeculation during cardiac development. $A$ : sagittal section through wild-type $(+/+)$ embryonic day 10.5 mouse heart. The ventricle (Vn) and atrium (At) are separated by the endocardial cushion (EC). The outer myocardial (My) muscle and inner endocardial (En) endothelium are readily distinguishable. Elaborate myocardial trabeculae (Tr) are lined by a layer of endocardial endothelial cells. $B$ : equivalent sagittal section through an $\mathrm{ErbB}_{4}(-/-)$ mutant heart. Note the complete absence of ventricular trabeculae with detached monolayer of endocardial endothelium (En), a slightly reduced endocardial cushion, and dilatation of both atrium and ventricle. RBC, clusters of red blood cells which are more abundant in the mutant heart as a typical feature of poor function. Similar pictures as in $B$ were obtained in neuregulin $(-/-)$ (Fig. 2 in Ref. 380) and $\mathrm{ErbB}_{2}(-/-$ ) (Fig. 4 in Ref. 308) mutant mouse hearts. [From Gassmann et al. (189).]

cardial alterations produced by null mutations in VEFG-A (209) and angiopoietin-1 or its receptor TIE-2 $(500,562)$.

Accordingly, just before myocardial trabeculation there seems to exist a critical interdependence between various signaling pathways, including VEGF, neuregulin, serotonin, and angiopoietin-1 growth factor pathways, with reciprocal signaling between the endocardial endothelial cells and the developing myocardium (19, 102, 574). These endocardial endothelial-myocardial interactions are highly specific and essential steps in normal cardiogenesis. If one of these signaling pathways is disrupted, the ventricle remains untrabeculated and most of the animals soon die in utero.

B) ENDOCARDIAL CUSHION DEVELOPMENT: VALVE FORMATION AND SEPTATION (FIG. 6, BOTTOM). I) Endocardial endothelial lineage. In the region of future cardiac valve formation, endocardial endothelial cells are transformed into mesenchymal cells which migrate into the cardiac jelly. Transformation into mesenchymal cells is accompanied by downregulation of the endothelium-specific PECAM-1 (299) and QH1 $(119,407)$ expression. It occurs during a narrow spatio- temporal window of intense programmed cell death, or apoptosis $(264,456,597,653)$. The resulting local mass of mesenchymal cells pushes the endocardial layer into the cardiac lumen and increasingly expresses smooth muscle $\alpha$-actin, necessary for cell motility and migration (119, 407), as well as urokinase, necessary for remodeling of the matrix and cell migration $(348,369,545)$. These protrusions are known as the endocardial cushions from which the cardiac valves and septa are formed $(302,616)$. Predictably, the cloche mutant heart in zebrafish, which lacks endocardium, lacks also cardiac cushions and valvuloseptal formation (549).

The spatially restricted subpopulation of endothelial cells destined to participate in endocardial cushion formation can be identified at an early phase in cardiac morphogenesis by the expression of JB3, a fibrillin-2related antigen (629), and of the "slug" transcription factor gene $(73,483)$. Thus it would be tempting to suggest that the JB3 immunoreactive and the slug immunoreactive endocardial cells constitute the same cell population (73) and that both JB3 and slug could constitute reliable 
markers of EE cells destined to transform into mesenchymal cells.

II) Myocardium-derived induction. Recent discoveries about the mechanisms underlying endocardial endothelial transformation into mesenchymal cells during endocardial cushion and valve formation have led to a better appreciation of the various endocardial-myocardial signaling pathways that participate in cardiac development. During cushion formation, the developing myocardium strongly expresses and secretes various EDTA-soluble (ES) messenger protein complexes, e.g., ES/130 protein and other so-called "adherons," which are required to induce endocardial endothelial-to-mesenchymal transformation $(140,471)$. This induction signaling process, which is elicited initially by the myocardium, is amplified in an autocrine fashion by additional expression of ES/130 in the endocardial endothelium and in the endocardial cushion tissue itself (471). Three members of these myocardium-derived induction protein complexes have been so far identified: fibronectin, transferrin, and kLAMP-1 (637). ${ }^{7}$ For the expression of at least two of these, i.e., fibronectin and kLAMP-1, the presence of retinoic acid (the major active metabolite of vitamin A) is essential. This may explain the great variety of congenital valvuloseptal deformations resulting from impaired mesenchymal cell formation in the endocardial cushion, associated with either maternal excess or deficiency in retinoic acid or vitamin A (46, 47, 129, 407).

III) Transforming growth factor- $\beta$. An understanding of the mechanisms by which cushion endothelial cells respond to this myocardial induction is fundamental to the understanding of growth responses during cardiac development $(339,409)$. Candidate molecules that have been implicated in the cushion transformation process are transforming growth factor- $\beta$ (TGF- $\beta$ ) and bone morphogenetic protein (BMP), among others. Virtually every cell in the body produces TGF- $\beta$ and has receptors for it. Of the three isoforms, TGF- $\beta_{1}$ and TGF- $\beta_{3}$ are expressed in the heart early during embryonic development, in endothelial, and mesenchymal cells, respectively. TGF- $\beta$ is increasingly expressed in cushion endothelial cells just before their transformation into mesenchyme, but also in the immediate subjacent myocardium (50). TGF- $\beta$ expression by the endocardium is under regulation of the ES proteins $(54,120,406)$.TGF- $\beta_{2}$ was only transiently expressed at the time of induction of the cushion in the cardiomyocytes underlying the regions in which the endocardial-mesenchymal transformation will take place (30). Absence of TGF- $\beta_{2}$ in TGF- $\beta_{2}$-null mice resulted in abnormal valve leaflets and septa that derive from endo-

${ }^{7}$ kLAMP-1, a 283-kDa protein identified using a monoclonal antibody against a fraction of soybean agglutinin. cardial cushion tissue. These TGF- $\beta_{2}$ knock-out mice die around birth.

TGF- $\beta$ binds to its receptors T $\beta$ R-I, T $\beta$ R-II, and T $\beta$ RIII (54) and interacts with other molecules such as betaglycan and endoglin. The latter two binding molecules are components of the TGF- $\beta$ receptor complex, which may modulate its biological action. Endoglin was found to be strongly but transiently expressed in endocardial endothelial cells, but not in the myocardium, during endocardial cushion formation (464). The receptors for TGF- $\beta$, $\mathrm{T} \beta \mathrm{R}-\mathrm{I}$, and $\mathrm{T} \beta \mathrm{R}$-II, as well as betaglycan, were found mostly on cardiac myocytes and were detectable only at low levels on endocardium. Transient upregulation of the various constituents of the TGF- $\beta$ receptor complex, and in particular of endoglin, is also an essential step in valve formation and ventricular septation.

Yet, there is no apparent cardiac malformation in single-knock-out mutant mice lacking either TGF- $\beta_{1}$ or TGF- $\beta_{3}$, which suggests pharmacological redundancy of TGF- $\beta$ in cushion formation even though TGF- $\beta$ is essential for cardiogenesis (409). BMP, as a member of the TGF- $\beta$ growth factor superfamily, is also expressed in the subjacent myocardium and acts synergistically with TGF- $\beta$ to initiate endothelial-to-mesenchymal transformation $(50,409,634,635)$.

Finally, there exists some interaction between the TGF- $\beta$ pathway with retinoic acid, the major active metabolite of vitamin A. The retinoic acid signaling pathway, noted above, plays an essential role at an earlier stage in the establishment of the primary heart tube and in the expression of induction protein complexes in the cardiomyocytes before cushion formation. Retinoic acid deficiency in mice resulted also in downregulation of TGF- $\beta_{1}$ (33), whereas retinoic acid treatment resulted in an increased expression of TGF- $\beta_{1}$ in endocardial and mesenchymal cells (346). Mice deficient in RXR- $\alpha$, one of the retinoic acid receptors, display hypoplastic cushion formation (208). TGF- $\beta$ would thus seem to act downstream of the retinoic acid signaling in cushion formation.

IV) Neuregulin. The neuregulin growth factor (NRG-1) pathway (discussed above) was shown to also play a specific and essential step in endocardial cushion formation through the $\mathrm{ErbB}_{2} / \mathrm{ErbB}_{3}$ receptor complex, which is expressed by the prevalvular mesenchymal cells adjacent to the neuregulin-expressing endocardium of the endocardial cushions (144). Null mutation in the $\mathrm{ErbB}_{3}$ receptor resulted in abnormal cardiac valve formation with subsequent congestive heart failure and death in utero.

V) Insulin-like growth factor I. Endocardial endothelium also expresses insulin-like growth factor I (IGF-I). IGF-I expression is highest in the endocardial endothelium, appears to decrease in the mesenchymal cells, and is absent in the myocardium (388). While having no apparent effect by itself, in contrast to NRG-1, IGF-I interacts 
with NRG-1 synergistically to promote expansion of the atrioventricular cushion tissue (226).

VI) Myocardial-derived hepatocyte growth factor. Myocardial-derived hepatocyte growth factor (HGF) may also participate in inducing and maintaining endothelialto-mesenchymal transformation, at least partly by upregulating the expression of urokinase in the endocardiumderived mesenchymal cells (545).

VII) Nuclear factor of activated T cells. The transcription factor NF-AT c $_{\text {c }}$ NF-AT ${ }_{2}$ ) (nuclear factor of activated $\mathrm{T}$ cells), known to participate in the immune system, was selectively expressed by endocardial endothelial cells near the endocardial cushion (111, 416, 472). Mutant NF-AT $\mathrm{C}_{\mathrm{c}}-/-$ embryos did not undergo outflow tract valve formation and underwent only partial ventricular septation with subsequent congestive heart failure and premature death of the embryo. The atrioventricular valves were either less severely affected (472) or normal (111) in these NF-AT ${ }_{c}-/-$ mutant embryos. NF-AT would appear to be involved in events that are essential for valve formation, but after, and independently of endothelial-to-mesenchymal transformation, when TGF- $\beta$, which is expressed at normal levels in NF-AT $\mathrm{A}_{\mathrm{c}}-/-$ heart, is no longer involved (472).VEGF was recently shown to play an important role also in valve formation by inducing NF-AT ${ }_{c}$ activation specifically in these valvular endothelial cells (495).

VIII) Neurofibromin. Another interesting development was the observation that neurofibromin (Nf1), which is transiently overexpressed in cushion mesenchymal cells, downregulates endothelial-to-mesenchymal transformation (301). Mutant mice deficient of Nf1 (Nf1 -/-) display a dramatic overabundance of spongy endocardial cushion tissue, with failure to condense and thin to form mature valve leaflets due to a lack of appropriate apoptosis in the $\mathrm{Nf1}-/-$ endocardial cushions in addition to increased proliferation (301). Subsequent compaction of the ventricular myocardium is also hampered, and often accompanied by ventricular septum defect and thinning of the compact myocardial layer, with death in midgestation.

IX) Platelet-derived growth factor. Another candidate receptor tyrosine kinase expressed in the transforming cushion tissue is the platelet-derived growth factor receptor $\alpha$-subunit (PDGF- $\alpha \mathrm{R}$ ) (301). PDGF- $\alpha \mathrm{R}$ can bind the secreted ligand PDGF-A which is expressed at high levels in the cardiomyocytes. This was initially believed to be indispensable (502), but PDGF- $\alpha \mathrm{R}$ knock-out mice did not show endocardial cushion defects (547).

X) Pre-B-cell growth-stimulating factor. A variety of other molecules including the chemokine Pre-B-cell growth-stimulating factor/stromal cell-derived factor-1 (PBSF/SDF-1) (405) are also expressed in the endocardial endothelium and seem to be involved in endocardialmyocardial signaling. Mutant PBSF/SDF-1-deficient em- bryos develop cardiac ventricular septal defects. As cushion and valve formation are slightly retarded but normal in these mutant embryos, these signaling pathways appear to have an essential but rather late developmental function. In situ hybridization revealed that PBSF/SDF-1 mRNA was expressed in the endocardium of the muscular septum, when the membraneous portion of the ventricular septum is to be formed, suggesting a key role of this chemokine in ventricular septum formation (405). Most mice lacking PBSF/SDF-1 died perinatally.

XI) Endothelin. A putative role of the endotheliumderived endothelin (ET) pathway has also been demonstrated. In the embryo, ET-1 mRNA is expressed in the entire endocardial endothelium around the time of cushion formation (296, 297). Moreover, although ET-1 mRNA and protein were also expressed in cardiomyocytes of rat embryonic hearts, immunostaining with anti-ET antibody was strongest near the endocardium (415). The mRNA for ET-converting enzyme (ECE-1) and for the $\mathrm{ET}_{\mathrm{A}}$ receptor were expressed both in the endocardial endothelium and subjacent myocardium (641). Yet, in neither ET-1- or $\mathrm{ET}_{\mathrm{A}^{-}}$ deficient mutant mice embryos nor after pharmacological suppression of $\mathrm{ET}-1 / \mathrm{ET}_{\mathrm{A}} / \mathrm{ET}_{\mathrm{B}}$ signaling was the induction of endothelial-to-mesenchymal transformation inhibited (87, 296, 408); in contrast, a large proportion of the ET-1deficient animals developed ventricular septal defects at a later stage (296). Hence, ET-1/ET ${ }_{\mathrm{A}}$ signaling appears not to be essential for initiating cushion formation but may play a role at later stages near the completion of the transformation process as well as during early compaction of the septum and ventricular wall. This is supported by the more severe cardiac defects observed after disruption of ECE-1 (440). ECE-2 mRNA is largely absent in the heart before these stages and seems to be expressed exclusively in the mesenchyme of the endocardial cushions (640). Most intriguingly, although neither single ECE-1 -/ - nor ECE-2 -/- mice display any detectable developmental defect in cushion formation, combined ECE-1 - - and ECE-2 - - - double null mice embryos exhibited abnormal atrioventricular valve formation, despite significant residual tissue levels of ET-1/ET-2. This suggests that ECE-2 may contribute to the production of mature ET-1 in situ in the endocardial cushion mesenchyme or that it would function in endocardial cushion by cleaving non-ET peptides essential for valve formation (640).

XII) NO. A similar rather late contribution of endothelium-derived NO in cushion formation has been suggested from experiments in mice lacking endothelial constitutive NO synthase (ecNOS). These ecNOS -/animals survived into mature animals but demonstrated a high incidence of bicuspid aortic valves; in the corresponding wild-type embryonic mice, ecNOS was localized to the cardiomyocytes and endocardial endothelial cells lining the developing valve leaflets (310). In another study 
on ecNOS -/- mice, a high incidence of atrial and ventricular septal defects, again suggesting cushion malformation, was observed, with death soon after birth (155). It may also be worth mentioning that ecNOS and inducible NO synthase (iNOS) were prominently but transiently expressed at a late phase in the myocardium of mice and rat embryos and in a model of embryonic stem cellderived cardiomyocytes during maturation into adult cells, becoming undetectable in these cardiomyocytes when terminally differentiated (43). As pharmacological inhibition of NOS resulted in a pronounced differentiation arrest of the cardiomyocytes, these observations would suggest that at some stage, corresponding probably to the late phase of cushion formation and early myocardial compaction, myocardium-derived $\mathrm{NO}$ could in addition to the endothelium-derived NO also play a role in cardiomyogenesis. That NO is probably essential for cardiomyocyte survival at these early stages of development is further supported by the observation that ecNOS-deficient mutant mice show increased cardiomyocyte apoptosis $(154,155)$. We (Andries, Sys, and Brutsaert, unpublished data) and others (594) observed that ecNOS expression in the EE in the developing rat heart occurred at a much earlier stage than ecNOS expression in MyoCapE, even when myocardial capillaries in the compact zone of the ventricular wall were already detectable by rat endothelial cell antibody (RECA) staining. During later compaction, ecNOS was present not only in EE but also in the developing MyoCapE and in coronary vessels within the compact myocardium, although absent from the cardiomyocytes (594).

XIII) Angiotensin. Mutant mice deficient of angiotensin type $1 \mathrm{~A}$ and $1 \mathrm{~B}$ receptor genes similarly survive normally in utero but may develop ventricular septal defects suggesting an important but late ontogenic role of angiotensin II (ANG II) and its $\mathrm{AT}_{1}$ receptor in cardiac development (590).

C) FORMATION OF COMPACT MYOCARDIUM (MYOCARDIAL COMPACTION). At a later stage during cardiac development, the outer myocardial layers of the embryonic heart become compact. Initially, the compact zone is only about two myocardial cells thick and is avascular. As compaction expands through cardiomyocyte proliferation to a thickness of three to four cells, angioblasts begin forming vascular tubes from the epicardium (581).

The compact zone comprises the outermost layers of the ventricular cavities and a major portion of the muscular septum. In birds, the septum arises from coalescence of trabecular sheets. In mammals in contrast, the septum has a compact arrangement from the onset (514). The ventricular septum is thus formed by two distinct processes: 1) through cushion formation with septation of the conotruncus and outflow tract and coalescence of trabeculae at the interventricular groove, and 2) through inward growth, or myocardialization (596), and compac- tion of the fused medial walls of the expanding ventricles forming the major muscular portion of the septum.

The molecular and functional phenotype of the ventricular compact myocardium differs distinctly from the ventricular trabecular component (169-171, 398). The maturing and differentiating trabeculated myocardium expresses specifically the cyclin-dependent kinase inhibitor p57Kip2, which is absent in the proliferating compact myocardium (278). Trabeculations, moreover, retain the characteristics of the primary myocardium. They express predominantly 1) atrial-specific isoforms of the contractile protein, compared with the ventricle-specific isoforms of the contractile proteins in compact myocardium; 2) atrial-like gap junctional $\mathrm{Cx} 40 \mathrm{mRNA}$ and protein rather than the ventricle-like Cx43 mRNA and protein in compact myocardium; and 3) a higher sarcoplasmic reticulum $\mathrm{Ca}^{2+}$-ATPase (SERCA2) and lower phospholamban (PLB) mRNA than in compact myocardium (170). As a result, trabecular myocardium has a quicker twitch contraction and faster impulse conduction (85) but shows a longer lasting relaxation. This performance pattern suggests that trabeculations may play an important transient role by driving contraction and impulse conduction during maturation when specialized conduction tissue and mature myocardial cells have not yet been fully formed.

Gene inactivation studies in mice have indicated that myocardial compaction is under the control of a great variety of growth signaling pathways and their myocardial-specific receptor genes, which apparently share a common molecular pathway necessary for compaction, such as retinoic acid (RA) (the major active metabolite of vitamin A) and the myocardial RA receptors (RAR, RXR) (46, 47, 258, 259, 514, 559), basic FGF (bFGF) (581) and its receptor tyrosine kinase (FGFR1) (381), TGF- $\beta 2$ (497), the nuclear protein jumonji (311), the FOG-2 gene as an essential developmental cofactor for GATA-4/5/6 (575), the $\alpha$-dystrobrevin gene (233), and probably many more pathways still to be discovered. ${ }^{8}$ Many of the known pathways have been shown to initiate or to enhance compaction, but some may counteract this process and serve as a suppressor of cardiomyocyte proliferation. Mutant

\footnotetext{
${ }^{8}$ In humans, noncompaction syndrome is a rare, congenital, idiopathic cardiomyopathy that is due to ventricular compact layer hypoplasia resulting from intrauterine arrest of compaction of the ventricular wall. It is characterized by prominent trabeculations and deep intertrabecular recesses within the left ventricle, sometimes affecting also the right ventricle and interventricular septum. Mortality and morbidity are high, following heart failure, thromboembolic events and ventricular arrhythmias (42, 80, 133, 245, 246, 248, 421). Nutritional deficiency (134) or premature differentiation (258) has been proposed as a possible cause. Noncompaction was observed, for example, in hearts from vitamin A-deficient embryos after maternal vitamin A deprivation (624). Similar myocardial noncompaction phenotypes have now been generated in mice gene-targeted for a variety of transcription factors, cell surface receptors and kinases $(164,229)$, erythropoietin and its receptor (628), and the FOG-2 gene as an essential developmental cofactor for GATA-4/5/6 (575).
} 
TGF- $\beta-/-$ mice, for example, develop a significant thickening of the ventricular wall and a loss of ventricular chamber volume, both of which result from cardiomyocyte hyperplasia (315).

I) Endothelin. Many genes that encode the transcription factors for initiating the thickening of the outer compact ventricular myocardium are expressed in the cardiomyocytes, but it is at present unclear whether additional signals from the endocardial endothelium, from the developing epicardial coronary vascular endothelium, or from the MyoCapE are also required $(581,582)$. At the onset of compaction, for example, mRNA for ECE- 1 and ET-1 are expressed both in the endocardial cushion tissue and in the endocardial endothelium of the ventricular cavities (640), and they have been shown to contribute to the compaction process of the ventricular wall and the large portion of the muscular septum. Kurihara et al. (296) observed hypoplasia of the compact zone of the ventricular wall, including severe ventricular septal defects, in the mutant ET-1-deficient embryos when they were treated also with the ET antagonist BQ-123 to eliminate the effects of circulating maternal ET. Intriguingly, an interventricular septum defect was observed in all the ECE-1 - /- mutant embryos, despite significant remaining ET-1 tissue levels (641).

II) Erythropoietin. Erythropoietin (EPO) is also an essential growth factor during compaction (628). Hearts from mutant EPO $-/-$ or EPO receptor (EPO-R) - /embryos suffered from ventricular hypoplasia, coupled to defects in the interventricular septum, due to a reduction in the number of proliferating cardiomyocytes in the septum and ventricular compact layer. EPO-Rs are highly expressed in endothelial cells (5) including in the endocardium, but not in the cardiomyocytes (628). Moreover, EPO has been shown to promote endothelial cell proliferation in vitro (121). It would, therefore, be feasible that EPO would trigger cardiomyocyte proliferation indirectly through its action on the endothelial cells, both in the endocardial endothelium of the trabeculated layer and in the cardiac endothelial cells of the proliferating myocardial capillaries in the expanding compact myocardial layer. An obvious potential candidate for this EPO-triggered endothelial-myocardial interaction at these two sites could be the EPO-induced release of ET-1. ET-1 is highly expressed in these two types of endothelial cells as is the $\mathrm{ET}_{\mathrm{A}}$ receptor on the cardiomyocytes at this later stage of development. Isolated cardiomyocyte cell proliferation and $\left[{ }^{3} \mathrm{H}\right]$ thymidine incorporation studies have indeed revealed a mitogenic action of EPO in cells isolated from EPO -/- mice, but not in cells from EPO-R -/animals. The involvement of MyoCapE is supported by the observation of epicardial detachment and lack of defined myocardial capillary structures in EPO $-/-$ and EPO-R -/- hearts (628).
III) $N R G-1$ and IGF. Interaction of the cardiac endothelium-derived growth factors NRG-1 and IGF-I synergistically induced substantial DNA synthesis of cardiomyocytes with significant growth and thickening of the ventricular compact zone (226). As pointed out by the authors, this is a most intriguing observation since NRG-1, when given alone to a whole mouse culture system, induced trabeculation of the ventricular wall but without a significant increase in the proliferation of cardiomyocytes, whereas IGF-I, while essential for cardiac growth in neonatal heart, had no apparent effect by itself on cardiac development.

D) CORONARY VASCULARIZATION. The embryonic heart of higher vertebrates is avascular until the myocardium becomes thickened through compaction. In human embryos, the first sign of definitive blood vessels was found to be localized in the subepicardial space of the apical interventricular incisura (228). These primitive coronary vessels are lined by a monolayer of (coronary) vascular endothelial cells which soon become physically continuous and contiguous with the endocardial endothelium. Hence, the development of a mature coronary circulation and the establishment of a myocardial capillary vascular plexus are relatively late events, the phylogenetic and embryological importance of which depends entirely on the ratio of compact-to-spongious myocardium. ${ }^{9}$ Recent studies support the hypothesis that coronary vascular growth during compaction is genetically induced by the myocardium (575) and somehow regulated, at least in part, by the rate and magnitude of myocardial growth (580). This is supported further by the observation that threshold levels of VEGF-A expression in the myocardium are required, not only for proper formation of endocardial endothelium as we have seen above, but also for the establishment of a myocardial vascular network $(72,214$, 586). Currently, however, the underlying regulation of embryonic coronary vasculogenesis (through in situ local formation) or angiogenesis (through outgrowth or branching of preformed vessels) are still not well under-

${ }^{9}$ The thick compact layer, with its prominent coronary vasculature, in the homeotherm birds and warm-blooded mammals relates to the high aerobic metabolic needs and concomitant requirements for more efficient cardiac pump performance. Comparative anatomy and physiology of poikilotherm animals show that the presence of coronary vessels in different fish species seems to be related to the weight and degree of compaction of the heart and to the degree of physical activity of the animal rather than to its phylogenetic position. For example, among teleost fish, the cardiac wall of the trout and of the tuna, both "athletic" fish, is made of two distinct layers, the internal spongy layer being supplied through diffusion from the intertrabecular spaces, while its external compact layer has a distinct coronary vascular supply. The heart rate of tuna can reach 120/min and systolic blood pressure can rise to $100 \mathrm{mmHg}$, ranking among the highest pressures recorded in fish. In contrast, the heart of smaller and "less athletic" teleost species, such as the Japanese medaka, has a very thin, entirely trabeculated wall which is exclusively supplied through diffusion from the cardiac chamber (see Ref. 6). 
stood (581, 582). Experiments with replication-defective retrovirus-mediated genetic tags (386) have, however, revealed vasculogenesis, rather than angiogenesis, as the mechanism of coronary vessel formation $(383,385)$.

Are endocardial and vascular endothelia derived from distinct cell lineages? From traditional embryological observations, the answer to this question has been unclear. Recent biochemical evidence, however, indicates that endocardial endothelial cells are distinct from coronary vascular endothelial cells, including MyoCapE (339, 383). For example, as noted above, expression of NF-AT is restricted to specified EE cells (111, 472). Other evidence that EE cells and MyoCapE are distinct is provided by the zebrafish mutant cloche, which lacks an endocardial tube but not blood vessel endothelium (549). The fact that cloche acts upstream of VEGF and its receptor Flk-1 (327) also indicates that the formation of EE and MyoCapE cells may be regulated through different signaling pathways. In further support of these functional differences is the observation that adult EE cells in culture tended to proliferate and to reach confluence significantly faster than cultured MyoCapE or coronary vascular endothelial cells $(9,371,373,417,418)$.

Accordingly, the emerging field of targeted gene manipulation has over the past 10 years strongly supplemented the contention that cardiac endothelial-myocardial interaction is a prerequisite for normal cardiac development, structure, and function. It has, moreover, resulted in a better understanding of some of the molecular mechanisms and cellular signals governing this interaction. This interaction involves a finely tuned series of molecular, morphological, and functional events that, if perturbed even slightly, can have dramatic consequences. Information from null phenotype experiments grows and as more molecular pathways are discovered and characterized, this overview will inevitably need updating. The extent to which these molecular pathways govern physiological or pathophysiogical cardiac adaptations in the adult heart remains to be elucidated (79).

\section{Mature and adult heart}

Most studies on the cardiac endothelial-myocardial signaling pathways discussed above have focused on their role in cardiac development rather than their regulatory role postnatally.

It is still generally believed that cardiomyocytes in the postnatal and adult heart are terminally differentiated, although recent reports suggest the contrary $(12,37,250)$.

Cardiomyocytes may, however, respond by hypertrophic growth to a wide array of stimuli, including mechanical and oxidative stress, as well as metabolic (hypoxia), neurohormonal, and growth factors. Many factors with myocardial growth-modulating properties have been shown to be released by adult cardiac endothelial cells.
The possibility that auto- or paracrine pathways including cardiac endothelial-myocardial interactions might modulate cardiac growth or gene expression during adaptive hypertrophy is intriguing.

That cardiac endothelial cells might be indispensable for myocardial growth in the adult was suggested by experiments with cardiomyocytes cocultured with endothelial cells. Only in the presence of cardiac endothelial cells could the cardiomyocytes maintain their adult phenotype; when vascular endothelial cells from aorta or fibroblasts were added, cultured cardiomyocytes continued to undergo dedifferentiation and reexpression of fetal proteins observed in long-term monoculture of adult cardiomyocytes (138). Endothelial cells stimulated also the secretion of atrial natriuretic peptide from atrial cardiomyocytes in coculture $(318,319)$. Reciprocal signaling from cardiomyocytes to MyoCapE has also been observed: MyoCapE cell growth was indeed significantly enhanced when cocultured with cardiomyocytes (414). Moreover, only in coculture with cardiomyocytes were MyoCapE able to express mRNA for both TGF- $\beta$ precursor and pre-pro-endothelin (ppET). In these coculture experiments, TGF- $\beta$ acted as an autocrine cytokine, increasing ppET mRNA and inhibiting the rate of MyoCapE cell proliferation (414). In other studies, expression of von Willebrand factor in MyoCapE was shown to be induced through a signaling pathway mediated by cardiomyocytedependent, platelet-derived growth factor $\mathrm{AB}$ heterodimer (PDGF-AB); only MyoCapE with PDGF- $\alpha$ receptors could transduce the signal for the expression of the gene for von Willebrand factor, whereas neighboring MyoCapE devoid of these receptors lacked this ability $(2,136$, 484). Finally, endothelial cell progenitors in mice embryo as well as differentiated endothelial cells from mice umbilical vein can differentiate into beating cardiomyocytes when cocultured with neonatal rat cardiomyocytes or when injected near the damaged area of the heart after occlusion of a coronary vessel, the latter transdifferentiation being independent from signaling molecules active in embryogenesis (91a).

A) ROLE OF PARACRINE ENDOTHELIAL-MYOCARDIAL SIGNALING. Endothelial-derived molecules such as $\mathrm{NO}, \mathrm{ET}, \mathrm{PGI}_{2}$, and ANG II may influence myocardial growth, as they are known to influence vascular smooth muscle growth. NOS and ET are expressed, albeit at negligibly low levels, in quiescent cardiomyocytes as well as constitutively expressed in cardiac endothelial cells of the normal adult heart. ecNOS expression in cardiac endothelium is highest in EE and only scant in MyoCapE (8). Whether endothelial or myocardial in origin, NO and ET may theoretically participate in growth responses in the adult heart. Cardiac endothelial dysfunction has for example been invoked to explain maladaptive growth responses during the progression of heart failure. 
I) NO. Many experimental and clinical observations support an antigrowth effect of NO in the adult heart, which may, at least in part, explain the beneficial effects of angiotensin converting enzyme (ACE) inhibitors on ventricular "remodeling" (68, 216, 220, 238, 247, 332, 345, $363,367,368,524)$. Their inhibition of bradykinin breakdown with enhanced bradykinin-induced NO release will contribute to their antigrowth, representing another example thereby of cardiac endothelial-myocardial signaling pathways. Bradykinin has a direct growth-stimulating effect on cardiomyocytes in monoculture, but an antigrowth effect which is critically dependent on the presence of endothelial cells in coculture, and in particular on the release of endothelium-derived $\mathrm{NO}$ and $\mathrm{PGI}_{2}$ (479, 480). Disruption of the bradykinin $\mathrm{B}_{2}$ receptor in mice leads to inappropriate cardiac hypertrophy with marked chamber dilatation and reparative fibrosis (142), an effect which was prevented by an ANG I receptor antagonist (142). Targeted disruption of the tissue kallikrein gene triggered dilated cardiomyopathy in mice (375), further supporting the view that kinins normally stimulate the release of $\mathrm{NO}$ and $\mathrm{PGI}_{2}$ through activation of bradykinin $\mathrm{B}_{2}$ receptors. Blunted basal activation of the NO (and $\mathrm{PGI}_{2}$ ?) pathway in $\mathrm{B}_{2}-/-$ knock-out mice would leave unbalanced ANG II-induced myocardial growth. The kallikrein kinin system may, therefore, be cardioprotective by counteracting the remodeling processes through its action on cardiac endothelial-myocardial signaling.

NO may act also as a molecular switch by promoting or counteracting growth actions of bFGF, VEGF, and TGF- $\beta$ in the adult heart $(433,643)$. Levels of ecNOS activity in the cardiac endothelial cells may thus be crucial to controlling growth and remodeling in the heart (17, 45, 200, 230, 288, 420, 433, 434, 654-656).

II) Endothelin. The role of ET in cardiac growth in the adult heart is less clear despite its known mitogenic potential. ET mRNA expression has been reported in cardiac endothelial cells (372), but not in normal cardiomyocytes in the normal adult heart (415). In DOCA saltinduced hypertensive rats with cardiac hypertrophy, ET-1 mRNA expression was enhanced in endocardial and coronary endothelial cells, including those in the small intramyocardial coronary arteries (306). ET was thought by the investigators of this study not to contribute to the hypertrophy since ET mRNA was not significantly increased in myocardial cells. We suggest that alternatively, the observed upregulation of ET in the cardiac endothelial cells could have sufficed to trigger hypertrophy of the myocardial cell. In a closely related model of hypertensive hypertrophy in the rat, right and left ventricular tissue levels of ET-1 were not augmented during the hypertrophic, nonfailing phase of the experiment (241), again suggesting alternative pathways for adaptive myocardial growth in the adult animal. Only at the transition from left ventricular hypertrophy to congestive heart failure were the myocardial tissue levels significantly increased, suggesting a role for myocardial ET-1 in maladaptive growth or tissue remodeling. Myocardial ET-1 tissue levels were also markedly increased only in close association with the deterioration of cardiac function following acute myocardial infarction and pressure overload (400). In another study, however, left ventricular hypertrophy in response to acute pressure-overload in cardiomyocyte-specific ET-1 knock-out mice was reduced despite preservation of coronary vascular endothelial ET-1 expression (403).

III) Angiotensin. Endothelial-myocardial signaling may in the adult heart also substantially contribute to the well-known growth-promoting properties of ANG II, much of which is believed to result from locally produced tissue ANG II $(123,284,441)$. The ACE that transforms ANG I in its active ANG II form is expressed in the endothelial cells in the heart, both in coronary vascular endothelium and in cardiac EE and MyoCapE, as well as in cardiomyocytes and in fibroblasts $(123,135,163,262$, 304). Moreover, cardiac overexpression of ACE in transgenic mice resulted in ultrastructural changes in MyoCapE and in a hypertrophic pattern of gene expression (451, 512). The growth response was however much smaller than in mice with cardiac-specific overexpression of angiotensinogen; as cardiac ANG II was not increased, this suggests at least in the mouse that $\mathrm{ACE}$ is not a limiting factor for cardiac ANG II formation (365). A substantial ACE-independent conversion into ANG II in the ventricles of the heart may occur through cardiac chymase, a large proportion of which is localized in cardiac interstitial and cardiac endothelial cells (593). The growth-promoting effect of ANG II on cardiomyocytes has been shown to involve auto- and paracrine release of ET-1 from the cardiac endothelial cells (239, 240, 467). Examples of interactive "cross-talk" between endothelium-mediated signaling pathways to adjacent cardiomyocytes continue to proliferate.

B) ROLE OF PEPTIDE GROWTH FACTORS. Targeted gene disruption experiments have provided valuable insight into the molecular basis of cardiac development and the indispensable role of endothelial-myocardial interactions in this process. Some genetic mutations have, however, been lethal. Early embryonic lethal mutations can thus prevent further analysis of their potential contribution to endothelial-myocardial signaling. The prominent roles of receptor tyrosine kinases in other organs suggest though that these signaling molecules may play critical roles also in the adult heart. The introduction of organ-restricted, or conditional, mutant animals will open new avenues to explore these possibilities.

I) Vascular endothelial growth factor. The fate of the VEGF signaling pathway in the adult heart has been under intensive investigation in view of its role in hypoxiainduced angiogenesis and its potential therapeutic usefulness in treating ischemic heart disease $(26,311,448,569)$. 
VEGF, also known as vascular permeability factor (160), is a potent, specific mitogen for endothelial cells, and it is devoid of mitogenic activity for other cell types (636). In the normal adult heart, both cardiomyocytes and MyoCapE cells express substantial amounts of VEGF mRNA (360, 534). Relatively smaller amounts of its receptor KDR/Flk-1 mRNA were detected in MyoCapE cells, but none in cardiomyocytes (360). Interestingly, the abovedescribed PDGF-AB/PDGF- $\alpha$ receptor signaling pathway from cardiomyocyte to MyoCapE, necessary for the expression of von Willebrand factor in some MyoCapE cells, also induced expression of VEGF and its receptor Flk-1 in these MyoCapE cells (136).

Flt-1 (VEGFR1), unlike Flk-1, is maintained at high levels in the differentiated endothelium of adult vascular tissues, implying a possible role in the cardiac endothelium of the adult heart, as for example in regulating their adhesion to one another or the extracellular matrix (167).

The first target of VEGF, secreted from cardiomyocytes, could be the MyoCapE cells. Whether the relatively high VEGF expression in normal adult cardiomyocytes might reflect effects other than neovascularization and endothelial migration remains an open question. The VEGF-KDR/Flk-1 signaling pathway could contribute to the many other processes resulting from an activated cardiac endothelial-myocardial interplay. VEGF can, for example, increase NO (654) and $\mathrm{PGI}_{2}$ (404) production and thereby influence myocardial remodeling and performance.

Might these speculations about MyoCapE-myocardial interactions be extended to EE-myocardial interactions in the adult heart? In adult hearts, EE contains specific receptor binding sites for recombinant human VEGF (probably VEGFR-1 or Flt-1) but not for the mature form of human VEGF-C (343). The recently discovered nontyrosine kinase receptor for VEGF, neuropilin-1 (NP-1), is also known to be expressed in EE cells as well as in cardiomyocytes (271).

The VEGF signaling pathway in neonatal and adult hearts can be upregulated or activated by hypoxia and ischemia $(26,218,300,317,321,536)$, cardioplegiareperfusion (579), the proinflammatory cytokines interleukin (IL)-1 $\beta$ (360) and tumor necrosis factor (TNF)- $\alpha$, and oxidative stress (84). IL- $1 \beta$, for example, may increase the expression of VEGF mRNA 3.6-and 2.4-fold in cardiomyocytes and MyoCapE cells, respectively, while a 3.0-fold increase of its receptor KDR/Flk-1 mRNA was observed in MyoCapE (360). Cardioplegiareperfusion in pig heart was associated with a fourfold increased expression of myocardial VEGF mRNA and a sixfold increase in Flk-1mRNA expression in the MyoCapE (579). Some or all of these factors may come into play in disease, to cardiac remodeling and angiogenesis. Interestingly, pulsatile mechanical stretch of iso- lated perfused hearts and of cultured rat cardiomyocytes induced rapid mRNA expression and secretion of VEGF and VEGF receptors, mediated probably in an autocrine fashion by the secretion of TGF- $\beta 1$ by cardiomyocytes and activation of intracellular signaling pathways including mitogen-activated protein kinase (MAPK) family members $(322,516,517)$.

II) NRG. The NRG-ErbB receptor signaling pathway, important in early cardiac development, continues to be expressed into adult life. NRG is expressed mainly by $\mathrm{EE}$ and MyoCapE cells. $\mathrm{ErbB}_{2}$ is expressed in cardiomyocytes and MyoCapE; $\mathrm{ErbB}_{3}$, however, is expressed only in MyoCapE, and $\mathrm{ErbB}_{4}$ only in cardiomyocytes $(181,305,652)$. Soluble NRG-1 (recombinant human glial growth factor 2 or rhGGF2) provoked a substantial increase in embryonic cardiac myocyte proliferation, as well as an increased survival and inhibition of apoptosis of cultured cardiomyocytes and could also induce hypertrophic growth in both neonatal and adult ventricular cardiomyocytes $(20,652)$. The cardiac mitogenic action of 5-HT via a $5 \mathrm{HT}_{2 \mathrm{~B}}$ receptor-mediated pathway is dependent on an intact neuregulin-ErbB2 signaling pathway in the embryonic and adult heart (411). The persistent expression of these proteins in the adult heart suggests that endothelium-derived NRG is essential for myocardial function and survival, another example of the indispensable role of cardiac endothelial-myocardial signaling for normal cardiac function.

III) Angiopoietin. A similarly persistent and indispensable expression of genes involved in cardiac endothelial-myocardial signaling into adult life is demonstrated for the angiopoietin-1/Tek/Tie growth signaling pathway. From late gestation and throughout the life of the animal, Tek/Tie receptors are continuously expressed in the adult vascular and endocardial endothelium where they support maturation, maintenance, and survival of endothelial cells $(438,461)$. Angiopoietin-2 mRNA levels in cultured adult microvascular endothelial cells are upregulated, e.g., by cytokines, by hypoxia, by VEGF $(353,422)$, and by ANG II (180). Whether this naturally occurring antagonist of angiopoietin- 1 is also expressed in cardiac endothelial cells in the in vivo adult heart waits, however, futher confirmation.

IV) $T G F-\beta$ and $b F G F$. The peptide growth factors, TGF- $\beta$ or bFGF, are abundantly expressed in neonatal and adult cardiomyocytes $(256,262,508,548)$. Whether these factors contribute to cardiac endothelial-myocardial signaling in the adult heart, similarly as during cushion formation in the embryonic heart, remains to be defined.

Accordingly, these observations highlight a paracrine role for neuregulin-, VEGF-, and angiopoietinmediated endothelial-myocardial communication in maintaining phenotype and survival of adult cardiomyocytes. It is likely that they contribute also to remodel- 
ing of the adult heart in response to hypertrophic signals. A physiological role in the normal adult heart remains to be determined.

\section{B. Cardiac Contractile Performance}

Following the seminal observations by Furchgott and Zawadski (186) that vascular endothelium controlled vascular smooth muscle contraction, it was natural to question whether EE might similarly influence myocardial contraction. This indeed proved to be the case. Selective damage of the EE in isolated cat papillary muscle modified the pattern of twitch contractions $(62,63)$. It resulted in an immediate and irreversible abbreviation of the isometric twitch, with earlier onset of tension decline and concomitant decrease in peak twitch tension (Fig. 1), with little change in the early contraction phase of the twitch or in maximal shortening velocity $\left(V_{\max }\right)$. This change in twitch pattern was unusual in that it differed from the effect of all other inotropic interventions except for that of shortening resting muscle length. The findings are now widely confirmed $(10,110,563)$. The effect of removing the EE was shown to be attributable to a reduced responsiveness of the contractile proteins to the intracellular $\mathrm{Ca}^{2+}$ concentration $\left(\left[\mathrm{Ca}^{2+}\right]_{\mathrm{i}}\right.$ ), interestingly, as is the comparable effect of shortening muscle length; a slight increase in $\left[\mathrm{Ca}^{2+}\right]_{\mathrm{i}}$ has also been demonstrated (110, $610)$.

Several groups have subsequently shown that it is not only the EE but also the endothelium in the myocardial capillaries (MyoCapE) which regulate the contractile state of subjacent cardiomyocytes (323, 414, 470, 543). This response has been ascribed predominantly to known auto- and paracrine signaling agents, released or activated by the cardiac endothelial cells, such as NO, ET, $\mathrm{PGI}_{2}$, and ANG II $(122,146,190,286,371,372,447,459,511,523,528$, $543,594,611,613)$. In relation specifically to the $\mathrm{EE}$, an active transendothelial physicochemical gradient for various ions, or blood-heart barrier, has been postulated (173) (discussed in sect. IIIC). With this concept of a blood-heart barrier in mind, any cardiac role for the putative vascular endothelium-derived hyperpolarizing factors $(153,603)$ has yet to be explained. Likewise, the potential participation of other endothelium-mediated signaling pathways, such as bFGF, VEGF, neuregulin, or angiopoietin, in modulating myocardial inotropic performance remains to be determined.

\section{Autocrine and paracrine signaling from cardiac endothelial cells}

Cardiac endothelial cells like all other endothelial cells express and release a variety of auto- and paracrine agents, which directly influence cardiac metabo- lism, growth, contractile performance, and rhythmicity. In the normal adult heart, cardiac endothelial cells produce NO through the expression of constitutive NOS (ecNOS or NOSIII), endothelin (ET) after conversion of pre-proET to pro-ET and into ET through the ECE, the eicosanoids, and prostacyclin $\left(\mathrm{PGI}_{2}\right)$, and transform ANG I into active ANG II.

The synthesis, secretion, and activities of these endothelium-derived substances are closely linked, interrelated, and interactive. Many of these agents modulate the actions of the other endothelium-derived agents on the same target cell, actions which may be mutually additive, synergistic, or inhibitory. They may even result in novel effects, not seen with either agent alone, and as determined by the milieu in which the interactions occur. It may therefore be simplistic to try and define their properties independently from one another. Too little attention is given to such interactions and interdependence. ${ }^{10}$ As a consequence, by merely focussing on one or only a few aspects of a single one of these paracrine factors, some of the issues become confused, and, perhaps more importantly, the total picture often gets lost. The emphasis to date on the role of NO, for example, or of ET or ANG II, may distort the overall picture if the contributory and interacting role of other agents to the biological outcome is insufficiently appreciated. With this caveat, it is nevertheless didactically necessary to focus on the specific expression, distribution, and activity of these agents independently in the first place, adding reference to their interactions and interdependence so far as is practicable.

Beyond the reciprocal interplay among the various endothelium-mediated auto-/paracrine signalings, a still higher scale of complexity in the in vivo intact heart may ensue from their interaction with other important cardiomodulatory pathways, such as the $\beta$-adrenergic or cholinergic pathways in the heart, atrial and brain natriuretic peptide activity, and circulating thyroid and aldosterone hormones. Some, such as aldosterone, are synthesized in cardiac endothelial cells and act in an autocrine manner to suppress NO production (149). Others, such as atrial natriuretic peptide, are synthesized by cardiomyocytes but functionally depend on the presence of cardiac endothelial cells for their secretion $(318,487)$ and their action on ventricular myocardial performance (379). For the purpose of this review, the

\footnotetext{
${ }^{10}$ In a Medline search of 1998-April 2002 publications, we found for the following key words: (heart or cardiac) and nitric oxide, 3,176 references; (heart or cardiac) and nitric oxide and endothelin, 243 references; (heart or cardiac) and nitric oxide and endothelin and prostacyclin, 36 references; (heart or cardiac) and nitric oxide and endothelin and prostacyclin and angiotensin, 11 references.
} 
"higher" interactions will be discussed only to the extent that they contribute to cardiac endothelial-myocardial interactions. The reader is referred to a number of excellent reviews $(22,212,281,445,524)$.

A) NO. The effects of NO on myocardial contraction, relaxation, and heart rate have been much studied. NO can be synthesized from L-arginine by three different NOS isozymes, two of which, the endothelial constitutive (ecNOS, NOSIII) and the neuronal (nNOS, NOSI) isoform, are constitutively expressed in physiological conditions, while the third, inducible isoform (iNOS, NOSII), is biosynthesized only after stimulation by various stressors and cytokines. The signal transduction pathways of the NO effects in the heart have been reviewed in detail $(165,263,364)$. Many of the actions of NO on cardiac performance are attributable to activation of myocardial soluble guanylate cyclase to produce cGMP, but some are cGMP independent. The underlying mechanisms are however still not fully understood $(23,165,364,524)$. Equally unclear is the question whether the observed positive or negative inotropic and lusitropic actions of NO are beneficial or detrimental to overall pump performance of the heart. NO does not seem to be essential for survival. Mutant mice lacking ecNOS survive without obvious detriment, and without upregulation of other NOS isoforms (595). "Compensatory" upregulation of other agents, such as prostaglandins, or atrial natriuretic peptide (212), undoubtedly take place. More subtle effects on cardiovascular efficiency, flexibility, and stability would not be identified in such experiment. And it could be said that the roles of NO are of such fundamental importance to evolutionary survival that they have necessitated the development of additional "back-up" mechanisms.

I) Nonuniform distribution of NO in the normal heart. The ecNOS is mainly present in both coronary vascular endothelium and cardiac endothelium $(8,511)$, but to lesser extent also in the cardiomyocytes $(25,156$, $515,619)$. The nNOS is present only in a subpopulation of intracardiac ganglia and nerve fibers in atrial tissue and in some perivascular nerve fibers of ventricular myocardium $(274,513)$. Expression of nNOS in cardiomyocytes as well as its physiological role is still largely under investigation (394). The adult heart does not normally express iNOS.

Although all endothelial cells in the heart express ecNOS, immunostaining experiments have shown that there is a considerable nonuniformity of ecNOS expression between endocardial (EE), arterial, capillary (MyoCapE), and venous endothelial cells, with strikingly more intense staining in endocardial and coronary arterial vascular endothelial cells (8). ecNOS is generally associated with the particulate fraction in endothe- lial cells, in particular with the Golgi complex ${ }^{11}$ and, with domains of the plasma membrane, the caveolae. ${ }^{12}$

The more intense ecNOS staining in EE and coronary arterial endothelium appeared to be associated with more intensely labeled and larger Golgi complexes. Double staining with ecNOS and Golgi 58k protein, a Golgi marker, demonstrated that ecNOS had colabeled the Golgi complex. Golgi size is probably a marker of synthetic activity so that these data suggest that coronary arterial endothelial and EE cells have a higher synthetic activity than do MyoCapE and venous endothelial cells. Heterogeneity was characteristic also for ecNOS labeling of the peripheral cell borders. The ecNOS-stained peripheral borders were distinct in EE cells, less distinct in venous endothelial cells, nearly absent in arterial endothelial cells, and not observed in MyoCapE. The peripheral ecNOS-stained bandlike structure in EE coincided with PECAM labeling in double-stained preparations. PECAM is known to label the whole depth of endothelial intercellular clefts (Fig. 4). Previous studies on cultured endothelial cells have demonstrated that an NO-induced increase of cGMP decreases paracellular permeability. NO production by the peripherally located ecNOS in EE and venous endothelial cells might thus be involved in the regulation of paracellular permeability. Immunostaining for caveolin-1 showed that peripheral borders of EE cells were nearly completely devoid of caveolin labeling. This suggests that enzymatic ecNOS activity in EE cells, in contrast to cardiomyocytes, might be associated with membrane components other than caveolin or with parts of the cytoskeleton.

Immunostaining of whole myocardial tissue showed rather weak cytoplasmic ecNOS labeling in MyoCapE where there were few Golgi complexes. Double-immuno-

${ }^{11}$ The Golgi complex consists of one or more stacks of cisternae surrounded by vesicles. It is the site of biosynthesis of glycolipids and of sugar moieties of glycoproteins. In endothelial cells, the Golgi complex is involved in the synthesis of various proteins, ranging from extracellular matrix components like collagen to more typical endothelial components like the intercellular adhesion molecule PECAM-1, the von Willebrand factor, and coagulation factor S. Hypertrophied Golgi complexes in endothelium are characteristic for embryological processes, for endothelial regeneration, and for dysfunctional endothelium in pathological conditions, such as hypercholesterolemia, endotoxin injury, chronic ethanol administration, and hydrostatic edema formation. The size of the Golgi complex is thus a marker for the functional status of endothelial cells.

${ }^{12}$ Caveolae are specialized invaginations of the plasma membrane. In endothelial cells, caveolae are enriched with caveolin-1 (the nonmuscle isoform of a coat protein of caveolae), $\mathrm{Ca}^{2+}$-ATPase, $\mathrm{G}$ proteins, and inositol trisphosphate $\left(\mathrm{IP}_{3}\right)$ receptors. Caveolin-rich microdomains in the plasmalemma are sites for endothelial cell NOS and other molecules involved in transduction. Caveolin also resides in the Golgi complex and appears to cycle between these two compartments. From experiments on caveolin-1 knock-out mice, there seems to exist an inverse relationship between caveolin-1 abundance and NO production $(157,473)$. In cardiomyocytes, caveolae typically stain for caveolin-3 but not for the caveolin-1 isoform. 
staining of the MyoCapE showed complete overlap of ecNOS labeling and labeling with RECA, an antibody that labels endothelium of the entire vasculature in all organs in the rat. MyoCapE was rather weakly labeled with diffusely distributed PECAM-1 staining (Fig. 4). Quantification of ecNOS mRNA in MyoCapE of canine heart by competitive PCR showed that, although the levels were higher than in coronary artery endothelium, "normalized" levels were somewhat lower for each individual endothelial cell (184). In some capillaries, thin PECAM-1 bands could be observed, suggesting the existence of thin, scarcely discernible peripheral cell borders (Fig. 4). Immunostaining for caveolin-1, however, was very intense in MyoCapE, where it was much stronger than in EE or arterial endothelium. Increased levels of caveolin-1 in MyoCap E were shown to exert cardioprotective effects against ischemia-reperfusion-induced injury, presumably via enhanced release of endothelium-derived NO (646). This latter interpretation is somewhat surprising, however, since caveolin- 1 has been generally recognized as an endogenous inhibitor of ecNOS activity in endothelial cells $(157,473)$.

The reasons for these differences in ecNOS distribution are still largely unknown. Experiments in cultured endothelial cells have demonstrated that ecNOS expression can be modulated by many things including shear stress, TGF- $\beta$, protein kinase $\mathrm{C}$, TNF- $\alpha$, oxygen, and the proliferative state. Differences in shear stress in the heart could explain the differential expression of ecNOS in arterial, capillary, and venous endothelial cells. But what about EE? Laminar fluid shear stress is probably not high along the surface of EE cells. Nevertheless, EE manifested almost equally strong ecNOS expression as in arterial endothelial cells. The EE surface might be more subjected to turbulent flow, but this type of flow does not increase NOS mRNA and NO release in cultured human umbilical vein endothelial cells. Mechanical strain of EE by three-dimensional changes of the inner wall during the cardiac cycle might influence ecNOS expression. Endothelial cells cultured on flexible substrates and subjected to cyclic strain have shown an increase in NOS mRNA, protein, and NO production. However, we did not observe significant differences in ecNOS expression between various areas of EE known to be subject to distinct differences in mechanical deformation during the cardiac cycle, e.g., the tendon end of right ventricular papillary muscles and the atrioventricular valves (8). EE cells covering these highly elastic structures are smaller and have a cytoskeletal organization that is different from that of other endocardial areas, but they did not show any consistent differences in ecNOS labeling or in the size of Golgi complexes. Freshly isolated endothelial cells from large porcine coronary arteries do express more ecNOS protein and produce more NO than do endothelial cells from resistance arterioles, although both are subjected to similar shear stress. More factors than shear stress are likely to influence the expression of ecNOS in cardiac endothelium. A direct kinin-mediated release of NO was observed in human MyoCapE, in response to several agents, such as ACE inhibitors, bradykinin, and $\alpha_{2}$-adrenoceptor agonists (267).

Several investigators have found ecNOS mRNA and protein expression in the cardiomyocytes (25, 34, 184, $263,515,619)$, possibly associated with caveolin-3, a muscle-specific isoform of a coat protein of caveolae (156). Nevertheless, the main physiological source of $\mathrm{NO}$ in normal, adult nonstressed cardiac tissue is probably ecNOS in EE and MyoCapE, while NO from the cardiomyocytes, unless activated, is probably negligible. No ecNOS could be demonstrated by immunostaining in the cardiomyocytes of normal adult rat and mice $(8,43,212)$ nor in mature stem cell-derived cardiomyocytes (43). Cardiomyocytes did not release NO in direct response to bradykinin or $\alpha_{2}$-adrenoreceptor agonists (267), further indicating that any cardiomyocyte-derived NO present is negligible (254) in basal, nonstressed physiological conditions. In contrast, exposure of the cardiomyocytes to $\beta$-adrenergic agonists increased endogenous NO production by almost fivefold, indicating upregulation of ecNOS in cardiomyocytes by $\beta$-adrenergic stimulation (254). Similarly, cGMP as a measure of NO activity increased by almost 10-fold in cardiomyocytes after stimulation with either bradykinin, unlike in the study above (267), or acetylcholine (270). Similarly, myocardial stretch may also participate in ecNOS activation (449).

Accordingly, there is considerable nonuniformity in the expression of ecNOS in cardiac endothelium. The intense ecNOS-labeling in EE (and coronary arterial) cells suggests greater ecNOS activity in these cells than in MyoCapE (and coronary venous endothelium). These two cell types probably account for most of the NO measurable in the effluent of in vivo heart or whole cardiac preparations. ecNOS is only faintly expressed basally in normal cardiomyocytes at mRNA levels but can be upregulated by a number of mediators. Hence, cardiomyocyte-derived NO does, in contrast to its role in many cardiac diseases as we will see later, most probably not normally participate in controlling overall structure and function of the normal adult heart in nonstressed physiological conditions. Expression of ecNOS (and possibly nNOS) in cardiomyocytes could, however, subserve an additional regulatory role through the autocrine production of NO in specific subcellular compartments (i.e., caveolae vs. sarcoplasmic reticulum membranes). It could, in addition, play a role in modulating cardiac function in response to specific stimuli or in conditions of myocardial stress (parasympathetic or adrenergic neurotransmitters, stretch, etc.).

II) NO and cardiac contractile performance. There appears to be a great diversity of, often conflicting, ac- 
tions of NO on myocardial contractile performance, depending on animal species, on experimental conditions, but most importantly on the experimental hierarchic level of investigation, whether it be the single isolated cardiomyocyte, the multicellular cardiac muscle preparation, or the in vivo intact heart. Single cardiomyocytes have been widely and usefully employed over the past 30 years, including by ourselves. Despite the theoretical advantages of this experimental model, e.g., in applying powerful molecular techniques to target specific signaling molecules in cardiomyocytes, the many, by the isolation procedure experimentally induced, uncontrolled artifacts may provide a somewhat distorted picture of reality. It exemplifies the limitations of the typical centripetal cardiomyocyte-oriented approach in cardiac research, not in the least because of their isolation from the obligatory cardiac endothelial cells (563) but also from neighboring cardiomyocytes. A positive inotropic response to $\mathrm{NO}$ was nevertheless observed in several studies on isolated cardiomyocytes $(269,282,283,607)$, whereas it induced a negative inotropic effect only at higher concentrations (52). Still, it remains uncertain whether any given response in single cardiomyocytes would be either beneficial or detrimental for cardiac function in the intact heart.

Somewhat more relevant data come from multicellular cardiac muscle preparations $(282,390,466,555)$. These show a typical dose-dependent biphasic inotropic response to NO. At the lowest levels, corresponding to endogenously generated NO, NO caused positive inotropic actions, whereas higher concentrations caused a consistent negative inotropic response. The response to increasing concentrations of cGMP is similarly biphasic (390). Inhibition of phosphodiesterase III by cGMP at low concentration elevates intracellular cAMP levels, which could account for the positive inotropy. cGMP at higher concentrations activates cGMP-dependent protein kinase, which will inhibit ATP synthesis and close voltage-gated calcium channels, which would account for the negative inotropic response. Alternative interpretations, including cGMP-independent mechanisms $(23,76,77)$, are possible, given the complexity of the various NO signaling cascades and their interactions (524). For similar reasons, in human ventricular muscle strips (166) although modulatory interactions of NO with autonomic nervous or other systems cannot be excluded, no direct inotropic actions of NO were observed.

An advantage of multicellular cardiac muscle preparations is that they allow for a full assessment of both systolic (contraction and relaxation) and diastolic (resting tension) properties of twitch contractions, neither of which can be ignored for a full evaluation of contractile performance. For example, NO at higher levels consistently causes an earlier onset of isometric twitch relaxation $(166,390,392,520,523,527,543)$. Depending on the often variable NO-induced effects on maximum rate of tension development, peak tension development may be either diminished, unaltered, or, exceptionally, even increased; if unaltered due to cancellation of opposing effects on twitch duration and rate, the intervention might, erroneously, be interpreted as having no effect.

Direct confirmation of inotropic effects of NO in the whole heart in vivo is difficult because of the confounding effects of changing loading, coronary flow or neurohormonal drive, and its interactions with the $\beta$-adrenergic and cholinergic pathways as well as with atrial natriuretic peptide activity in the heart $(212,524)$. Several studies have, nevertheless, provided indirect evidence for a minor positive inotropic action $(92,207,273,283,458,556)$. The presence in vivo of the above-mentioned biphasic in vitro effect of NO on inotropism, i.e., positive at low and negative at high NO concentrations, has been exemplified in transgenic mice overexpressing ecNOS (54a). In this study, NO was found to be positively inotropic in spontaneously beating hearts from wild-type mice, whereas hearts overexpressing ecNOS in the cardiomyocytes had reduced basal inotropy that was partially reversed by NOS blockade. More consistent is the in vivo observation that NO induces an early onset of ventricular relaxation, thereby enhancing ventricular relaxation, early rapid filling, and diastolic compliance (11, 205, 206, 344, 445-447). In many cases, this effect may be accompanied by a slight decrease in peak systolic pressure despite the often unaltered rate of pressure development and unaltered ejection properties $(344,445,524)$. Part of this effect is attributable to a NO-induced reduction of preload and afterload.

Such apparently negative inotropic effects may be regarded by many as potentially detrimental; rather, they should be considered as potentially beneficial to cardiac function (Fig. 8), acting as a compensatory feedback, when the physiological effects on contraction duration of enhanced ventricular preload and/or afterload are superimposed on the pathological prolongation of contraction duration in ventricular hypertrophy, especially if tachycardia intrudes on diastolic filling time (64, 65, 445, 524). Interestingly, Pinsky et al. (450) have demonstrated that there is a cyclical release of NO in the beating heart, most marked subendocardially, which peaks at the time of ventricular relaxation and early rapid filling. These appropriately timed, brief bursts of NO release would provide for important beat-to-beat modulation of ventricular relaxation, early filling, and diastolic coronary perfusion. The subendocardial localization would suggest EE cells as its major source.

Accordingly, NO seems to exert a dual action on myocardial contractile performance. In the lower concentration range, corresponding to endogenously generated NO through ecNOS, NO exerts a slight positive inotropic action, contributing to maintenance of basal contractile performance of the heart under physiological conditions. 

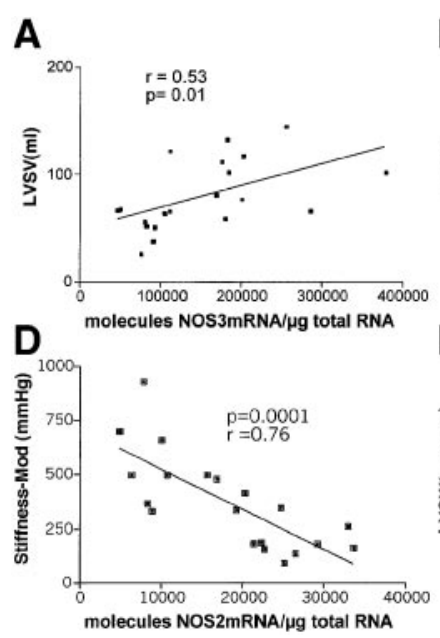

B

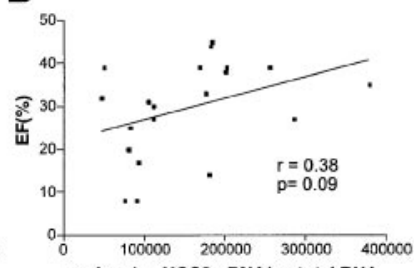

E

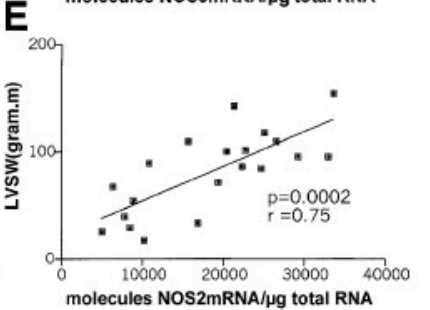

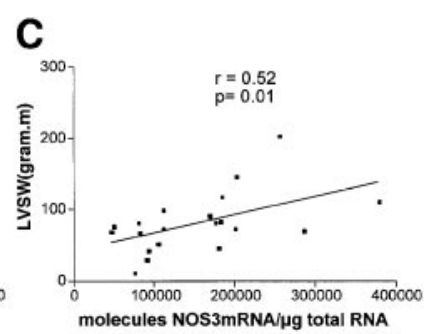

FIG. 8. Nitric oxide (NO) has beneficial effects on cardiac contractile performance in vivo in humans. Higher tissue levels of endothelial constitutive NO synthase (ecNOS) (NOS3 mRNA) $(A-C)$ or inducible NO synthase (iNOS) (NOS2 mRNA) ( $C$ and $D$ ) in endocardial-myocardial biopsies in patients were accompanied by an increase in left ventricular stroke volume (LVSV) $(A)$, ejection fraction $(\mathrm{EF})(B)$, and left ventricular stroke work (LVSW) ( $C$ and $E$ ) as well as by a decrease in stiffness-modulus $(D)$. The endomyocardial biopsies as a source for measuring tissue NOS3 mRNA (and partially for NOS2 mRNA) levels suggest EE as its major source. [Modified from Heymes et al. (227) and Paulus et al. (444) and kindly provided by Walter Paulus.]
At higher NO levels, a negative response in terms of peak contractile performance is consistently observed, as may occur with activation of iNOS or pharmacological doses of NO donors. More important, surely, are its effects on the onset of ventricular relaxation, both in beatto-beat optimization of pump function and coronary perfusion, and particularly as a compensatory mechanism to aid ventricular filling when this is prejudiced by disease. These effects on the timing of onset of ventricular relaxation, when normalized for heart rate, constitute one of the most fundamental modulators of cardiac systolic function $(64,65)$. By delaying the onset of relaxation, the heart will prolong and sustain cardiac work output during systole as part of heterometric autoregulation (Starling adaptation) in conditions of increased volume or pressure loading. Conversely, an earlier onset of ventricular relaxation, as seen with $\mathrm{NO}$, will favor ventricular relaxation, rapid early filling, diastolic compliance, and diastolic coronary perfusion, which would be particularly beneficial as a compensatory mechanism against ventricular overloading or in conditions of maladaptive hypertrophy and/or tachycardia. Interactions of NO with ET, $\mathrm{PGI}_{2}, \mathrm{ANG}$ II, $\beta$-adrenergic and cholinergic drives, atrial natriuretic peptide, and aldosterone must also be considered $(22,212$, $314,445,524)$.

III) NO and cardiac metabolism. In the normal as well as the failing human heart, endogenous and exogenous NO decreased myocardial tissue oxygen consumption $(337,588)$. Suppression of NO production had previously been shown to increase myocardial oxygen consumption in awake dogs $(38,531)$, although this was not confirmed in other models $(130,489,490,532)$. Inhibition of oxygen consumption by NO was documented also in noncontracting cardiac muscle slices (632). NO was produced almost exclusively by MyoCapE. Endothelial NO production and its inhibition of myocardial oxygen consumption were increased by ACE inhibition (650), acting probably through an increase in bradykinin levels
(452). Bradykinin-induced reduction in myocardial oxygen consumption was abolished in ecNOS knock-out mice (337). The ability of NO to lower myocardial oxygen consumption suggests a potentially cardioprotective mechanism of NO, perhaps in part due to the above decrease in cardiac loading and in cardiac systolic work output leading to increased myocardial metabolic efficiency (530); presumably also by influencing substrate (free fatty acids vs. glucose) utilization $(118,475,567)$ or by regulating mitochondrial metabolism (557). NO release from the MyoCapE and EE could thus directly regulate local myocardial metabolism. NO can reversibly compete with oxygen for a common binding site on cytochrome- $c$ oxidase, inhibiting electron transfer to oxygen (86). It has recently been shown in cultured cardiomyocytes (which do not normally produce NO in nonstressed conditions) that cytokine-induced NO production through expression of iNOS or exogenous NO delivery by an NO donor, lower energy, i.e., ATP production and myocardial contractility, through inhibition of the mitochondrial iron-sulfur reductases (572).

B) ET. Since its discovery in 1988 by Yanigasawa et al. (642), the role of ET in cardiovascular physiology and pathophysiology seems indisputable. In the heart, ET has been shown to be involved in cardiac development, growth, and remodeling, as discussed above, and also in the control of cardiac contractile performance and rhythmicity. Endothelial cells, including cardiac endothelial cells at the EE and MyoCapE, are its major source in the normal heart, and cardiomyocytes are its primary target. ET is thus likely to be a key player in the indispensable role of cardiac endothelial-myocardial interaction. Its supportive and protective role in normal cardiac function has been well recognized.

I) ET expression in the normal heart. ET is a 21amino acid peptide synthesized and released from endothelial cells. In the normal heart, ET-1 mRNA is expressed in EE (372), and ET-1 release has been demonstrated 
from EE cell culture (372) and from EE in isolated cardiac muscle $(107,146)$. ET-1 is also expressed in coronary vascular endothelium and in MyoCapE, and its release has been demonstrated in the venous effluent of isolated beating hearts (366). ET-1 is not normally expressed in normal, postnatal cardiomyocytes $(306,415)$. Cardiac tissue displays a high density of $\mathrm{ET}_{\mathrm{A}}$ and $\mathrm{ET}_{\mathrm{B}}$ receptors on cardiomyocytes, conduction tissue, and coronary vascular and EE cells (100, 243, 395). In pathophysiological conditions, a number of nonendothelial cells in the heart, including cardiomyocytes, come also to synthesize ET-1 in response, for example, to myocardial stretch, ANG II, and norepinephrine (400).

II) ET and cardiac contractile performance. ET is one of the most potent positive inotropic agents known $(326,366,372)$. Its inotropic action resembled the characteristic positive inotropic effect induced by the cardiac endothelium $(372,459,611)$ and has been partly explained by a similarly enhanced affinity of the contractile proteins to calcium (613), secondary to changes resulting from activation of the sarcolemmal $\mathrm{Na}^{+} / \mathrm{H}^{+}$exchanger (286). Similarly as for the contractile effects of NO, the inotropic response to ET largely depends on the type of experimental cardiac preparation that is being considered. For example, ET-1 was $\sim 60$ times mote potent in single cardiomyocytes than in papillary muscles (571). The more balanced physiological inotropic response to ET in the latter multicellular preparation may be due to integrated regulatory mechanisms involving in addition various noncardiomyocytal cell types, as e.g., cardiac endothelial cells. A physiological role for ET in cardiac contractile performance of the normal in vivo heart appeared uncertain because it would enhance myocardial oxygen consumption by virtue of its potent inotropic action, while at the same time decreasing oxygen supply through its powerful coronary vasoconstrictive action. It has however been demonstrated that ET binds stoichiometrically in vivo to its receptor (179). Such a binding feature predicts that under basal ET secretion, ET would act in an autocrine way by directly binding to the $\mathrm{ET}_{\mathrm{B}}$ receptor on the endothelial surface, thereby stimulating the release of $\mathrm{NO}$ and $\mathrm{PGI}_{2}$ in an autocrine manner $(116,341)$, rather than directly promoting myocardial inotropy through $\mathrm{ET}_{\mathrm{A}}$ receptors on the cardiomyocytes. The ET-1-induced release of NO could then affect the binding of ET-1 to its receptors and alter ET-1-stimulated $\mathrm{Ca}^{2+}$ mobilization and rearrangement of the cytoskeleton in the endothelial cells. Similar autocrine interactions of ET may also exist with endothelium-derived $\mathrm{PGI}_{2}$ (116) and ANG II (105). McClellan et al. (366) proposed ET storage and release from cardiac endothelial cells as a supportive cardioregulatory mechanism. Low ET concentrations may have important protective activities in the normal heart. For example, ET reversed acidosis-induced negative inotropic and lusitropic effects, without increasing intracellular calcium as occurs with most positive inotropic agents (611). ET is capable also of opposing the arrhythmogenic effects of catecholamines $(244,426)$ (see sect. IIIC). Endothelial release of ET during cardiac ischemia could thus act as a local auto- and paracrine hormone that directly counters these detrimental actions of the catecholamines.

C) $\mathrm{PGI}_{2}$. Endothelial cells, including cardiac endothelial cells, synthesize and release several eicosanoids in response to a wide variety of hormonal, chemical, immunological, and physical stimuli $(74,193)$. An important reciprocal interaction between $\mathrm{PGI}_{2}$ and $\mathrm{NO}$ on myocardial relaxation has been demonstrated. Compared with the effects of NO on cardiac contractile performance, those of endothelium-derived eicosanoids have, however, received relatively little attention.

I) Distribution of eicosanoids $\mathrm{PGI}_{2}$ and $\mathrm{PGE}_{2}$ in the normal heart. Cyclooxygenase (COX) plays a key regulatory role in prostaglandin synthesis. It occurs in both constitutive (COX-1) and inducible (COX-2) isoforms. COX-1 is constitutively expressed in all endothelial cells in the heart and is believed to provide cytoprotective effects (626). COX-2 is nearly undetectable under normal physiological conditions but is inducible in endothelial cells and in macrophages involved in inflammation. In the heart, COX-1 content has been found to be twice as high in the endocardial zone compared with the myocardium and was confined mainly to the endothelial cell fraction (53). EE from isolated cardiac valves (289), as well as valvular EE in culture (354), produce substantial amounts of $\mathrm{PGI}_{2}$. Mebazaa and co-workers $(371,373)$ have reported abundant production of $\mathrm{PGI}_{2}$ and $\mathrm{PGE}_{2}$ from cultured $\mathrm{EE}$ cells from right and left bovine ventricles, with EE production of $\mathrm{PGI}_{2}$ being $\sim 10$ times higher than of $\mathrm{PGE}_{2}$ and exceeding 19-fold and 34-fold the $\mathrm{PGI}_{2}$ release from vascular endothelial cells in aorta or pulmonary artery in response to shear stress and hypoxia, respectively (373). Shear stress-induced and hypoxia-induced release of 6keto-PGF $\mathrm{PG}_{1 \alpha}$ (a stable metabolite of $\mathrm{PGI}_{2}$ ) from $\mathrm{EE}$ was significantly suppressed by treatment with $17 \beta$-estradiol (the major circulating form of estrogen in females) treatment (476). 17 $\beta$-Estradiol similarly decreases ET-1 release from coronary vascular endothelium (and MyoCapE?) (615). Physiological increments of transmural pressure also significantly augment $\mathrm{PGI}_{2}$ release from porcine cultured EE cells, with values about three times higher in left ventricular than in right ventricular EE (417, 419). Vascular endothelial cells from coronary artery and aorta did not respond to increased transmural pressure, in contrast to the response of the pulmonary artery, which was similar to that of right ventricular EE cells. Interestingly, brief incubation with platelet-derived growth factor or TGF- $\beta$ was sufficient to stimulate the release of 6-keto$\mathrm{PGF}_{1 \alpha}$ from cultured EE from pig heart, whereas cultured vascular endothelial cells from pig coronary artery re- 
quired longer incubation time to release 6 -keto- $\mathrm{PGF}_{1 \alpha}$ (417).

These studies suggest that EE is a greater source of $\mathrm{PGI}_{2}$ than the vascular endothelium of major arteries, including the coronaries. But what about MyoCapE? MyoCapE cells isolated from rabbit heart synthesize and release 6-keto-PGF ${ }_{1 \alpha}$ and $\mathrm{PGE}_{2}$, the two major products of the COX pathway (193-195). In absolute values, however, significantly more (about 5-6 times) $\mathrm{PGI}_{2}$ was released from EE than from MyoCapE (417). Moreover, contrary to the 10 times higher $\mathrm{PGI}_{2}$ than $\mathrm{PGE}_{2}$ in $\mathrm{EE}$, this study showed that $\mathrm{PGE}_{2}$ release from MyoCapE exceeded that of $\mathrm{PGI}_{2}$ by $\sim 10$-fold. Why $\mathrm{PGE}_{2}$ and not $\mathrm{PGI}_{2}$ is the major eicosanoid produced by MyoCapE and why this ratio is opposite in EE is an intriguing question. In agreement with previous studies were the significant lower fractions of $\mathrm{PGI}_{2}$, and more so of $\mathrm{PGE}_{2}$, in vascular endothelial cells from major coronary artery.

II) Endothelium-derived eicosanoids and cardiac contractile performance. The possible inotropic actions of prostaglandins and their mainly cAMP-mediated underlying mechanisms are unresolved. Responses to $\mathrm{PGI}_{2}$ and $\mathrm{PGE}_{2}$ range from increased inotropy $(1,98,150,255,260$, $376,389,397,477,553)$, to no effect $(93,255)$, to negative inotropy (510). In vitro studies on isolated papillary muscle have helped to explain some of these inconsistencies (392). With the use of activators and inhibitors of endogenous eicosanoids and of $\mathrm{NO}$ in different combinations, it became clear that the inotropic action of eicosanoids and NO were reciprocal; stimulation of endogenous prostaglandin (mainly $\mathrm{PGI}_{2}$ ) release abolished the inotropic effect of NO, in particular on the onset of relaxation, while demonstration of a $\mathrm{PGI}_{2}$-induced positive inotropic (contraction-prolonging) effect was dependent on inhibition of NO synthesis.

Accordingly, $\mathrm{PGI}_{2}$ and $\mathrm{NO}$ interact to regulate cardiac contractile performance, by their opposing effects on the onset of myocardial relaxation. It has long been recognized that $\mathrm{NO}$ and $\mathrm{PGI}_{2}$ (and to a smaller extent $\mathrm{PGE}_{2}$ ) share a number of important properties and that their synthesis and release from endothelial cells are often coupled (74) through continuous cross-talk between the NOS and COX pathways $(193,225)$, although their underlying subcellular mechanisms await further clarification. Their mutual actions on the target cardiomyocyte are closely linked, largely through the effects of $\mathrm{PGI}_{2}$ and $\mathrm{NO}$ in establishing cAMP-to-cGMP ratios rather than absolute intracellular concentrations of cAMP or cGMP. Experimental as well as clinical evidence generally favors a supportive, protective role of $\mathrm{PGI}_{2}$ on cardiac function. Treatment with COX-inhibiting drugs is often not well tolerated, perhaps particularly in patients with preexisting cardiac endothelial dysfunction as in cardiac failure.

D) ANG II. Most effects of ANG II on cardiac growth and contractile performance are now thought to result from locally produced rather than from circulating ANG II (135, 599). In the normal heart, ANG II is synthesized locally through both ACE and an ACE-independent chymase pathway, both of which are expressed predominantly in coronary vascular and cardiac endothelial cells. Cardiac ACE is expressed also in cardiomyocytes and in fibroblasts (123). Evidence from in vitro and in vivo studies supports the view that ANG II produced by cardiac tissue may be important for normal cardiac contractile performance (377, 631). ANG II generally exerts a positive inotropic effect $(18,115,178,279)$, by increasing the rate of rise of the contraction phase and delaying the onset of relaxation (377), although the delayed onset of relaxation is often neglected in analyses of myocardial contractile performance. There are, however, conflicting reports of its action, ranging from a positive, to a negative, or to no inotropic response, depending on animal species or experimental conditions.

The inconsistent findings may reflect the many interactions of the cardiac synthesis, release, and activity of ANG II with the bradykinin-NO $(479,480,605)$ and PGI $_{2}$ (377, 479) pathways, as well as with ET-1. ANG II and ET-1 are cosecreted from cultured MyoCapE, for example, under basal as well as activated state, and they elicit synergistic effects on the heart (298). Their respective receptors on cardiomyocytes are also coupled, through similar G proteins, so that their intracellular signaling pathways may be similar. ET-1 mediates many of the ANG II effects, and vice versa. ANG II stimulates the expression of ppET-1 mRNA and protein in normal cardiomyocytes (83). The ANG II-induced stimulation of the anion $\mathrm{Cl}^{-}-\mathrm{HCO}_{3}^{-}$exchanger activity on myocardial performance is also mediated through endogenous ET-1 (105).

\section{Role of peptide growth factors}

Experiments in noncardiac tissue preparations provided circumstantial evidence that peptide growth factors may also have auto- and paracrine effects on cardiac function, other than on cardiac growth. VEGF, for example, has indeed been shown to increase intracellular calcium in vascular endothelial cells of coronary arteries and to affect vascular tone through the expression of ecNOS (290, 654) and of COX (404), resulting in potent endothelium-dependent $\mathrm{NO}$ - and $\mathrm{PGI}_{2}$-mediated vasodilatation. NO release from vessels could similarly be increased through activation of the bFGF/FGFR-1 signaling pathway (232).

Only preliminary data are presently available about a possible role for peptide growth factors in the performance of the adult heart, and it would be premature to review this subject. In years to come, we anticipate an overwhelming flow of new data and novel concepts on cardiac function, emerging from these signaling pathways, and further exemplifying, expanding, and clarifying 
our conjecture of the indispensable role of cardiac endothelial-myocardial interactions. Notably, MyoCapE of both fetal and adult heart abundantly express and release biologically active parathyroid hormone-related peptide (PTHrP), the expression of which is significantly upregulated by increased blood flow rate or by hypoxia, independently of the NO pathway $(504,505)$. PTHrP (but not PTH) exerts a positive inotropic, chronotropic, and lusitropic effect in adult ventricular cardiomyocytes, which do not themselves express the hormone. Little is so far known of the physiological and pathophysiological roles and underlying mechanisms of action of PTHrP in the cardiovascular system. The cardiac endothelium-derived PTHrP-mediated inotropic response would thus appear to be another example of cardiac endothelium-derived paracrine modulation of ventricular function.

\section{Cardiac Rhythmicity}

Rhythmicity of the heart is an intrinsic property of the specialized cardiac conductive tissue and terminal Purkinje fibers, indeed of every individual cardiomyocyte. Disturbances in excitability or conduction of one of these are a direct cause of arrhythmias. In the adult heart, the network of terminal Purkinje fibers lies just underneath and in close proximity to the endocardial endothelial surface, suggesting that their development, growth, and function might be directly influenced by EE.

\section{Differentiation of terminal Purkinje fibers: role of cardiac endothelium}

Purkinje fibers are the terminal, predominantly subendocardial, ramifications of a branching system of fiber bundles that emanate from the atrioventricular node through the bundle of His. Purkinje fiber cells are larger than the surrounding cardiomyocytes. To date, little is known about the mechanisms that regulate their differentiation and distribution patterning. Studies in early embryonic hearts before compact myocardium and specialized conduction tissue are fully formed suggest that the trabeculated layer of cardiomyocytes may drive contraction and impulse conduction at this stage, until they disappear as a distinct, functional component of the adult ventricle (398).

The genetic and molecular basis of how and why, depending on animal species (544), some cardiomyocytes from the primary myocardium differentiate into conducting Purkinje fibers has remained poorly understood (398, 503). Equally unresolved is whether endothelial-myocardial interactions are prerequisite for this important embryonic phenotypic transformation and whether such potential interactions continue to control excitability, conduction, and rhythmicity in the adult heart. Combined genetic, molecular, and functional and morphological ev- idence seems to favor the view that the inner layer of trabecular myocardium (including much of the interventricular myocardium) is a separate transcriptional domain, distinct from the compact myocardium (169), and from which the phenotypic transformation of some cardiomyocytes into the entire terminal ventricular conduction system may arise (398). It is appropriate here to acknowledge, in all humility, the careful observations by Tawara (573) in the beginning of the 20th century, on the basis of which these current molecular biological views could have been anticipated.

It is of interest to note the occurrence of irregular heartbeats in NRG-1 -/-, $\mathrm{ErbB}_{2}-/-$, and $\mathrm{ErbB}_{4}-/-$ mutant embryos. These arrhythmias may be due to conduction disturbances as a result of deficient trabeculation, i.e., the putative site of origin of the terminal conduction system. The partial or complete heart block in mutant (retinoic-X receptor- $\alpha$ ) RXR- $\alpha-/-$ embryos may, similarly, be ascribed to impaired trabeculation and compaction $(258,559)$. Needless to remind that the embryonic trabecular layer contains cardiomyocytes and EE cells in almost equal number and that both cell types, or their interaction, could contribute to the early transdifferentiation of some cardiomyocytes into terminal Purkinje fibers.

In the maturing embryonic heart, Purkinje fibers continue to be derived from localized recruitment of differentiated, beating cardiomyocytes alongside the developing coronary arterial bed (382). This recruitment of Purkinje fibers thus coincides with the early vasculogenic process and begins in cardiomyocyte subpopulations juxtaposed specifically to developing coronary arteries, but not veins (201). This spatiotemporal relationship suggests an inductive role of developing arterial coronary vessels in recruiting cardiomyocytes to transform into Purkinje fibers. Whether vascular endothelium in the smaller intramyocardial arteries and MyoCapE, or their interaction with subjacent cardiomyocytes, are involved in this transformation process and in the subsequent branching pattern of the peripheral Purkinje fiber network has yet to be established $(202,384)$. It may be relevant that the endothelium-derived ET signaling pathway has, near the completion of compaction, been shown to play an important role in the conversion of some cardiomyocytes into Purkinje cells (202). The cardiac failure seen in mutant ECE-1 -/- embryos (641) may accordingly be attributable to deficient development of the Purkinje system leading to conduction failure with bradycardia and cardiac arrest (408).

\section{The endocardial endothelium as a blood-heart barrier}

Before the initial observation that EE modulates the performance of subjacent myocardium (62), data relating 
to any physiological role of the EE in the adult heart were sparse. The existence of a blood-brain barrier (203, 485, 568 ) led us by analogy to consider the hypothesis that EE could perhaps, in addition to the release of paracrine mediators, establish a transcellular physicochemical gradient across the entire EE monolayer and thereby influence cardiac function (55). This possibility was supported by the striking feature in our original experiments of the abruptness with which the typical contractile twitch response to selective EE damage appeared, whichever damaging technique was used, be it Triton immersion, mechanical abrasion, a burst of high-frequency ultrasound, or exposure to a flow of dry air (Fig. 1) (110). None of the endothelium-derived factors exogenously added could prevent or reverse this abrupt response, nor could simultaneous pharmacological blockade of the NO, ET, and $\mathrm{PGI}_{2}$ pathways reproduce it (109). Obviously, breaching a barrier would be very unlikely to cause such an immediate, stable, and irreversible response if this were to act simply by enabling washout from the myocardium of diffusible endothelium-derived substances (in particular in respect to ET with its long half-life). It could however reflect an acute perturbation of the subendocardial ionic milieu. Apart from these speculations, however, we disposed at the time of only a few morphological features and of hardly any functional evidence to support this conjecture (61). In the meantime since 1989, convincing experimental evidence for the existence of an EE bloodheart barrier, or BHB, has however been gathered. The latter term was initially coined in 1995 by Dr. Paul Fransen in our laboratory (173) to strengthen the analogy with the blood-brain barrier concept.

The blood-brain barrier is the best-studied endothelial barrier. It is relatively impermeable to ions, amino acids, small peptides, and proteins. A unique feature is its high transendothelial electrical resistance of $\sim 1,500$ $2,000 \Omega \cdot \mathrm{cm}^{2}$ compared with other vascular endothelia $\left(6-25 \Omega \cdot \mathrm{cm}^{2}\right)$ (28). In the brain, the ionic composition of the extracellular fluid is controlled by transendothelial transport of ions. The asymmetric distribution of ion channels, pumps, and transporters between luminal and abluminal membrane of the brain capillary endothelial cells results in a transendothelial net $\mathrm{Na}^{+}$transport from blood to brain and $\mathrm{K}^{+}$transport from brain to blood. This generates a specific ionic imbalance beween the blood and the cerebrospinal fluid, which is well suited to maintaining optimal conditions for brain cell signaling. Highly excitable tissues such as the neurons necessitate high interstitial $\left[\mathrm{Na}^{+}\right]$to enable a rapid upstroke of their action potential and a low interstitial $\left[\mathrm{K}^{+}\right]$to increase membrane excitability. In the heart, the subendocardial terminal Purkinje fiber network and its adjacent myocardium are also highly excitable tissues, the ionic homeostasis of which being vital to cardiac function. Alterations in the extracellular concentrations of $\mathrm{Ca}^{2+}, \mathrm{K}^{+}, \mathrm{Na}^{+}, \mathrm{Mg}^{2+}, \mathrm{Cl}^{-}$, and
$\mathrm{HCO}_{3}^{-}$have profound effects on rhythmicity and on the mechanical performance of cardiac muscle. It is, therefore, essential that the ionic environment of the Purkinje fibers and adjacent cardiomyocytes be well controlled. The maintenance of a putative transendocardial electrochemical potential difference would, however, require a strong gradient for certain ions as well as a selective boundary barrier (basal lamina?) to prevent ionic leaks through myocardial capillaries and into the postcapillary coronary venular sink. Given the leaky nature of MyoCapE, any modulation of the interstitial ionic homeostasis by the EE is likely to be confined to the immediately subendocardial myocardial layers, which include the terminal Purkinje fiber network and the dense subendocardial neural plexus.

What evidence do we have that the EE would function as a BHB?

A) MORPHOLOGICAL FEATURES OF THE BHB. EE cells form a thin monolayer of closely apposed cells with complex interdigitations and extensive overlap at the junctional edges (Fig. 3). This would be consistent with the possession of unique permeability properties. Tight junctions are present and always located at the luminal side of the intercellular clefts between EE cells. At this luminal side, the glycocalyx is better developed than at the basolateral side below the tight junctions. EE cells thus display distinct morphological asymmetry. For further details about these specific morphological features of the EE, we refer to the sections on cardiac endothelial morphology (see sect. $\mathrm{III} B$ and Figs. 3 and 4 ).

Electrophysiological measurements of capacitive currents, together with dye-spreading fluorimetry, showed that EE cells were not only electrically coupled, but also dye-coupled through gap junction-like connections (172, $173,176)$. The presence of gap junctional coupling between EE cells has been substantiated by the expression of several connexins (Cx43, $\mathrm{Cx} 40, \mathrm{Cx} 37)$ at the border zone and intercellular spaces of the overlapping EE cells (Fig. 4, bottom). Gap junctions contain intercellular pores that permit charged ions (e.g., $\mathrm{Ca}^{2+}$ ), second messenger molecules (e.g., inositol 1,4,5-trisphosphate), and small metabolites to pass quickly between adjacent EE cells, resulting in an electrical and diffusional continuum or conduit (39). The evidence thus suggests that the endocardial endothelium should be considered as a syncytium with strong electrochemical coupling between EE cells.

Gap junctional connections between cardiac endothelial cells and cardiomyocytes have not been demonstrated (6). On rare occasions, myofibroblast-like cells were observed in the subendocardium to simultaneously touch EE cells and subjacent cardiomyocytes, but the functional role of these contacts has not been established (6). The absence of morphological junctions between $\mathrm{EE}$ and cardiomyocytes would not of course exclude an electrotonically propagated influence of the EE syncytium on 
excitability and conduction in closely subjacent cardiomyocytes and Purkinje fibers.

The EE syncytium can be likened functionally to one big cell with a very large membrane surface area; in comparison, the smaller cluster of the subjacent terminal Purkinje fiber network and dense subendocardial neural plexus can be functionally modeled as single small cells. Any electrical change in the membrane of the big cell will be electrotonically propagated to the small cell; electrotonic propagation in the reverse direction would, however, be negligible. Similarly, electrotonic effects emanating from the high capacitive cardiomyocytal syncytium could propagate to the EE, Purkinje network, and neural plexus. Experimental proof is lacking, but it would follow that EE cells, although "electrically silent" in the sense that they do not display regular action potentials, would depolarize to $E_{\mathrm{Cl}}$ and repolarize to $E_{\mathrm{K}}$ concomitantly with each action potential of the closely adjacent cardiomyocytes $(59,173)$. The syncytial character of $\mathrm{EE}$ is essential to the establishment of a putative barrier with unidirectional transcellular transport of ions. It would moreover serve to amplify $(39,496)$ the release of endotheliumderived paracrine substances, and it well suits the above suggested sensor function for the EE.

B) ELECTROPHYSIOLOGICAL FEATURES OF THE BHB. EE cells are in fact highly active electrically. Electrophysiological studies reveal the presence of a large number of membrane ion channels (inwardly rectifying $\mathrm{K}^{+}$channels, $\mathrm{Ca}^{2+}$-activated $\mathrm{K}^{+}$channels, background $\mathrm{Cl}^{-}$and cation channels, volume-activated $\mathrm{Cl}^{-}$channels, stretch-activated cation channels) and, at least, one carrier-mediated transporter $\left(\mathrm{Na}^{+}-\mathrm{K}^{+}\right.$-ATPase $)(173,175,177,231,307$, 351). The asymmetrical luminal versus abluminal (350) localization of ion channels and of $\mathrm{Na}^{+}-\mathrm{K}^{+}$-ATPase suggests a net transcellular transport of ions from the blood to the cardiomyocytal interstitium, and vice versa, by passive diffusion through ion channels and by active carrier-mediated transport. Transendocardial electrical resistance values of $50-80 \Omega \cdot \mathrm{cm}^{2}$ in near-confluent monolayers of cultured porcine right ventricular EE have been recorded (P. Fransen, unpublished observations). Although probably underestimated, they were still two to five times higher than in other endothelia $\left(6-25 \Omega \cdot \mathrm{cm}^{2}\right)$ (28), consistent with our conjecture that $\mathrm{EE}$ functions as an active barrier between the circulating blood and the cardiomyocytal interstitium. The combined application of electrophysiological techniques, Western blot, and RTPCR in porcine cultured EE cells and of immunostaining in ultrathin rat ventricular cryocoupes demonstrated copious expression of $\mathrm{Na}^{+}-\mathrm{K}^{+}$-ATPase, predominantly of the $\alpha_{1}$-type and typically confined to the luminal membrane of the EE cells (174) (Fig. 9). This asymmetrical configuration could account for net transport of $\mathrm{Na}^{+}$from the heart to the blood and of $\mathrm{K}^{+}$from the blood to the heart. A lower interstitial $\mathrm{Na}^{+}$in the heart would appro- priately provide for electrical stability (in contrast to brain, where a higher interstitial $\mathrm{Na}^{+}$rather favors excitability).

Accordingly, there is growing functional morphological and electrophysiological evidence to support the concept of EE as an active BHB. Apart from its role as a paracrine-mediated regulator of myocardial performance, particularly in the right ventricle, an active EE BHB could be of key importance for the overall ionic homeostasis of the interstitial milieu surrounding the adjacent excitable myocardium, the immediately subjacent terminal Purkinje fiber network, and the dense subendocardial neural plexus $(330,359,644)$. Interaction of $\mathrm{EE}$ with the latter two structures and its role in controlling conduction and rhythmicity await experimental evidence. It appears likely though that all EE cells act in solidarity as a functional syncytium to achieve a complex, finely tuned, auto- and paracrine-mediated physicochemical barrier between the circulating blood and subjacent cardiac tissue. Such would create optimal conditions for coordinated responses, as is necessary for a sensor device within the endothelial system.

\section{Cardiac endothelium and rhythmicity of the heart}

Evidence that cardiac endothelium might be involved in the control of rhythmicity first came indirectly from studies on the effects of prostaglandins on arrhythmias occurring during reperfusion of temporarily coronary-ligated dogs (90). These pointed to an antiarrhythmic action of endothelium-derived prostacyclin $\left(\mathrm{PGI}_{2}\right)$ and a proarrhythmic effect of platelet-derived thromboxane $\mathrm{C}$ actions, which were ascribed largely to the coronary vasodilating and vasoconstricting effects of $\mathrm{PGI}_{2}$ and thromboxane, respectively. A direct stabilizing action of $\mathrm{PGI}_{2}$ on rhythmicity, however, was also demonstrated in the dog (16). Subsequent studies have emphasized the important role of cardiac endothelium in suppressing arrhythmias $(430,436)$. The antiarrhythmic $(89,618)$ action of ACE inhibitors, for example, was ascribed to inhibiting the bradykinin breakdown with subsequent release of the antiarrhythmic $\mathrm{NO}$ and $\mathrm{PGI}_{2}$, rather than to inhibition of angiotensin (331, 333, 435, 436, 578, 606). These beneficial effects could be attenuated by treatment with bradykinin receptor antagonists or inhibitors of the COX and L-arginine-NO pathways (436). It was also shown that pronounced cardiac endothelial dysfunction preconditioned for severe arrhythmias (219). The underlying mechanisms involved in the antiarrhythmic effects of endotheliummediated $\mathrm{NO}$ and $\mathrm{PGI}_{2}$ remain incompletely understood $(219,437)$. Various possible mechanisms may be put forward. They could, for example, involve better balancing of the myocardial cAMP-to-cGMP ratio by optimizing relative $\mathrm{NO}$ and $\mathrm{PGI}_{2}$ activities $(3,24)$; normally, the latter messengers are closely linked, for $\mathrm{NO}$ stimulates $\mathrm{PGI}_{2}$ 
A

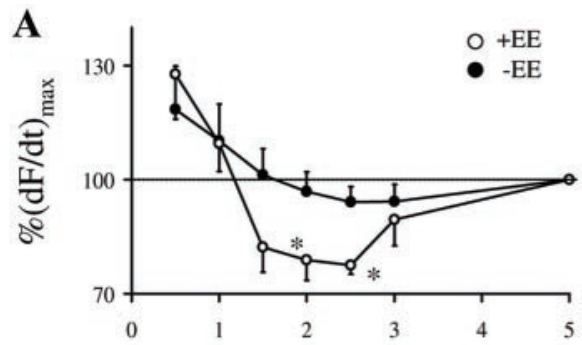

external $\mathrm{K}^{+}(\mathrm{mmol} / \mathrm{L})$

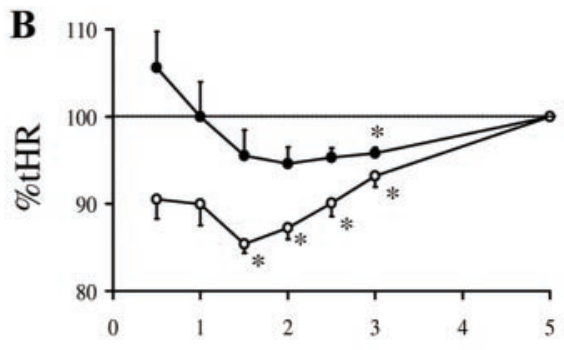

external $\mathrm{K}^{+}(\mathrm{mmol} / \mathrm{L})$

C

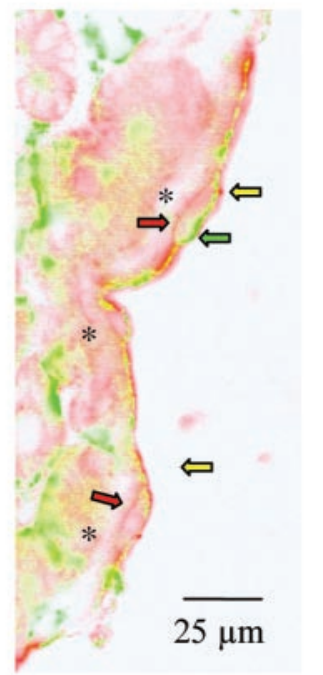

D
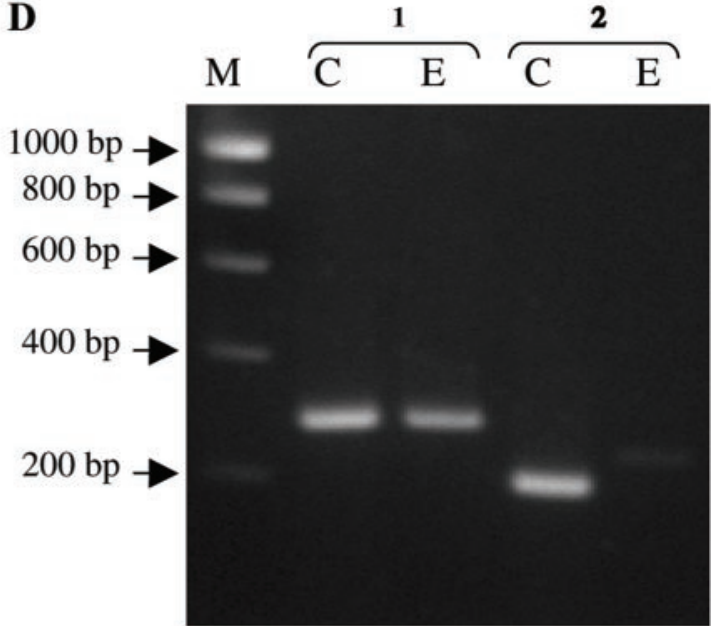

FIG. 9. Asymmetrical distribution of $\alpha_{1}-\mathrm{Na}^{+}-\mathrm{K}^{+}$-ATPase in endocardial endothelial cells. $A$ and $B$ : inhibition of $\alpha_{1}-\mathrm{Na}^{+}-\mathrm{K}^{+}$-ATPase by lowering extracellular $\mathrm{K}^{+}$concentration $\left(\left[\mathrm{K}^{+}\right]_{\mathrm{o}}\right)$ in cardiac muscle with $(+\mathrm{EE})$ and without $(-\mathrm{EE})$ endocardial endothelium. Peak rate of isometric twitch force development $(\mathrm{dF} / \mathrm{d} t)_{\max }(A)$ and time from stimulus to half-isometric twitch relaxation tHR $(B)$ are shown, as a function of $\left[\mathrm{K}^{+}\right]_{\mathrm{o}}$ in isolated papillary muscles from rabbit right ventricle. Parameteres were determined in muscles with the endocardial endothelium removed (-EE, solid symbols) and muscles with intact endocardial endothelium ( $+\mathrm{EE}$, open symbols) $(n=7)$ and expressed as percentage of baseline value at 5 mM $\left[\mathrm{K}^{+}\right]_{\mathrm{o}}$ in $-\mathrm{EE}$ and $+\mathrm{EE}$ muscles, which was taken as $100 \%$. All data are expressed as means $\pm \mathrm{SE}$. The stimulation frequency was $36 /$ min. Note the different scaling of the $y$-axis. Repeated-measures ANOVA was performed on the raw data (after logarithmic transformation for homoscedasticity) to analyze the effects of $\left[\mathrm{K}^{+}\right]_{\mathrm{o}}$ and the influences of an intact EE. Differences were considered statistically significant when $P<0.05$. $*$ Significant difference from the baseline value at $5 \mathrm{mM}\left[\mathrm{K}^{+}\right]_{0}(P<0.05) . P$ values for statistical comparison of $\left[\mathrm{K}^{+}\right]_{0}$ curves between $+\mathrm{EE}$ and $-\mathrm{EE}$ muscles were 0.025 for $(\mathrm{dF} / \mathrm{d} t)_{\max }$ and 0.0005 for tHR, indicating that inhibition of the $\alpha_{1}$-isoform of the $\mathrm{Na}^{+}-\mathrm{K}^{+}$-ATPase in EE at [K $\left.\mathrm{K}^{+}\right]_{\mathrm{o}}$ of 5 to $2 \mathrm{mM}$ causes a significant negative inotropic effect. $C$ : double immunostaining of cryostat sections of rat heart for PECAM (red) and $\alpha_{1}$-isoform of the $\mathrm{Na}^{+}-\mathrm{K}^{+}$-ATPase (green). PECAM outlined endocardial endothelial cells especially at sites of cellular overlap and also stained the abluminal side of cells as seen at the nuclei (arrows, asteriks). $\alpha_{1}$-Isoform staining was present at the luminal side of EE cells only, which was evident at the side of the cell nuclei (green arrows). Overlap between $\alpha_{1}$-isoform and PECAM staining is indicated by yellow, which is absent at the abluminal side of EE cells (yellow arrows). $D$ : RT-PCR of $\alpha_{1}$ - and $\alpha_{2}$-subunit isoforms of $\mathrm{Na}^{+}-\mathrm{K}^{+}$-ATPase in isolated rabbit cardiomyocytes (C) and rabbit cultured EE cells (E). M, molecular weight marker. Marker fragment lengths are shown on the left. The $\alpha_{1}$ - and $\alpha_{2}$-primers amplify a 289-bp and 205-bp fragment of rabbit $\alpha_{1^{-}}$and $\alpha_{2}$-subunits, respectively. The $\alpha_{1}$-isoform of the $\mathrm{Na}^{+}-\mathrm{K}^{+}$-ATPase was present in cardiomyocytes and EE cells, whereas the $\alpha_{2}$-isoform was present in myocytes only and not in EE cells. [Modified from Fransen et al. (174) and kindly provided by Paul Fransen.]

production directly by activating COX $(493,537)$. It could also involve modulation of cardiac endothelial and myocardial $\mathrm{Na}^{+}-\mathrm{K}^{+}$-ATPase activity $(3,357)$. The latter mechanism could partly explain why digoxin-induced arrhythmias are suppressed by endothelium-released NO, for by elevating myocardial cGMP, NO would indeed suppress the digoxin-induced increase in myocardial cAMP (455). Similarly in experiments on mice, it was shown that NO derived from ecNOS activation in single cardiomyocytes suppressed ouabain-induced arrhythmias through increased cGMP (292). Additional indirect mechanisms would operate through reduction of the arrhythmogenic activity after $\beta$-adrenergic activation in the heart (151,
524). NO also accelerates diastolic depolarization of the sinoatrial node (44), exerting a positive chronotropic effect that can itself be antiarrhythmogenic by overdrive suppression. That, in some experimental conditions of ischemia-reperfusion-induced arrhythmias, the NO pathway may appear to be beneficial and, in other ones, detrimental, is not really surprising (524).

The actions of endothelium-derived ET on cardiac rhythmicity are even more complex and unpredictable in their outcome. As we noted earlier, in the normal heart ET-1 mRNA is expressed exclusively in coronary vascular and cardiac endothelial cells (372), while $\mathrm{ET}_{\mathrm{A}} / \mathrm{ET}_{\mathrm{B}}$ receptor mRNA are expressed both in endothelial cells and 
cardiomyocytes, and interestingly, also in the atrioventricular conducting system (395).

Electrophysiological studies with voltage-clamped isolated cardiomyocytes suggest that ET has cardioprotective, "membrane stabilizing" properties; it inhibits the protein kinase A-dependent chloride current (244, $425,426)$ whose conductance is induced by catecholamines through $\beta$-adrenergic receptors. By shortening the action potential duration, it thereby counteracts the simultaneously increased L-type $\mathrm{Ca}^{2+}$ current. Inhibition of this current by ET would thus tend to counteract the potentially detrimental arrhythmogenic effects of increased plasma levels of catecholamines. The cardioprotective effects of ET-1 were recently endorsed by the observation that ET-1 could also counter $\beta$-adrenergic agonist-induced apoptosis in single, isolated cardiomyocytes (15).

Contrary to these in vitro studies in single cardiomyocytes are the manifest proarrhythmogenic effects of ET in vivo. ET may as a potent vasoconstrictor elicit arrhythmias secondary to coronary vasoconstriction (35, 565, 566), although observations that ET-induced ventricular arrhythmias may precede overt evidence of myocardial ischemia have led to suggestions that it could also have a direct arrhythmogenic effect (35, $565)$. On the other hand, reperfusion of ischemic rat hearts in the presence of ET-1 did not cause the expected reperfusion-induced increase in inositol 1,4,5trisphosphate $\left(\mathrm{IP}_{3}\right)$; given the proarrhythmogenic potential of $\mathrm{IP}_{3}$, ET-1-induced inhibition of $\mathrm{IP}_{3}$ generation during myocardial reperfusion would represent a novel antiarrhythmic mechanism (242, 627). Accordingly, given the many contradictory reports on the arrhythmogenic properties of ET-1, which may vary depending on pathophysiological concomitant events, anti-ET-1 therapy should be established with caution. As with NO, the multiple mechanisms of action both of ET and of arrhythmogenesis predict unpredictability of outcome.

One last point concerns the question whether endothelium could be involved in the electrical control of the heart other than through its active barrier properties (for the EE) or auto- and paracrine mechanisms. It was recently demonstrated that protein constituents of the glycocalyx in the MyoCapE could be specifically involved in the coronary flow-induced control of cardiac rhythmicity by the cardiac endothelium: antibodies against the endothelial surface proteins, $\alpha_{\mathrm{V}} \beta_{5}$-integrin and sialyl-Lewis glycan, depressed the dromotropic (rhythmicity-related) but not the inotropic effects of coronary flow-induced stress, whereas the vascular cell adhesion molecule (VCAM)-1 antibody had no effect on the dromotropic but enhanced the inotropic response to flow (486).

\section{CARDIAC ENDOTHELIAL DYSFUNCTION: ROLE IN THE PATHOGENESIS OF CARDIAC FAILURE}

Over the past two decades, we have witnessed a profound shift in the conceptual paradigms we apply to the syndrome of cardiac failure. Regardless of its etiology, it is generally progressive. It involves the recruitment of many compensatory mechanisms, such as cardiac dilatation and hypertrophy, as well as neurohormonal, cytokine, and endothelial cell activation. These cardiac and extracardiac adaptations can, however, become maladaptive and may eventually fail, leading to the overt clinical syndrome of cardiac failure. Maladaptation and failure are characterized by hemodynamic abnormalities, neurohormonal imbalance, cytokine overexpression, and endothelial dysfunction. The emerging pathophysiological picture of this multifactorial and progressive syndrome is one of failing "complexity," rather than of failure of a single organ, a single cell, molecule, or gene (108).

\section{A. Endothelial Activation and Dysfunction}

The terms endothelial activation and endothelial dysfunction (Fig. 10) are widely used but ill defined. Endothelial activation was introduced to describe changes in endothelial phenotype as part of physiological adaptative response to various possible injuries or stressors. These phenotypic changes include alterations in the cytoskeleton, the signaling cascades, and transcription factors, with rearrangement of gene expression $(21,106)$. The wide spectrum of phenotypic changes observed suggests that there may be numerous degrees of different, often overlapping, states of endothelial activation, extending as one continuous spectrum into manifest endothelial dysfunction. Endothelial dysfunction implies pathophysiological dysregulation and has been applied to conditions where endothelial activation is deemed to have become inappropriate, or maladaptive, or permanent. Why in some forms cardiac endothelium normalizes again while in others becomes or remains dysfunctional needs further investigation. Endothelial dysfunction may, in extreme cases, moreover lead to overt structural injury, and eventually to endothelial necrosis and frank denudation.

The clinical literature on endothelial (dys-)function relates mostly to impaired, endothelial NO production and bioavailability. The term endothelial dysfunction was introduced for conditions in which endothelium-mediated NO release in response to acetylcholine, bradykinin, substance $\mathrm{P}$, or serotonin, and the expected vasodilation had become deficient, compared with the response to the direct smooth muscle-mediated vasodilatatory response to adenosine or to various so-called NO donor substances, 


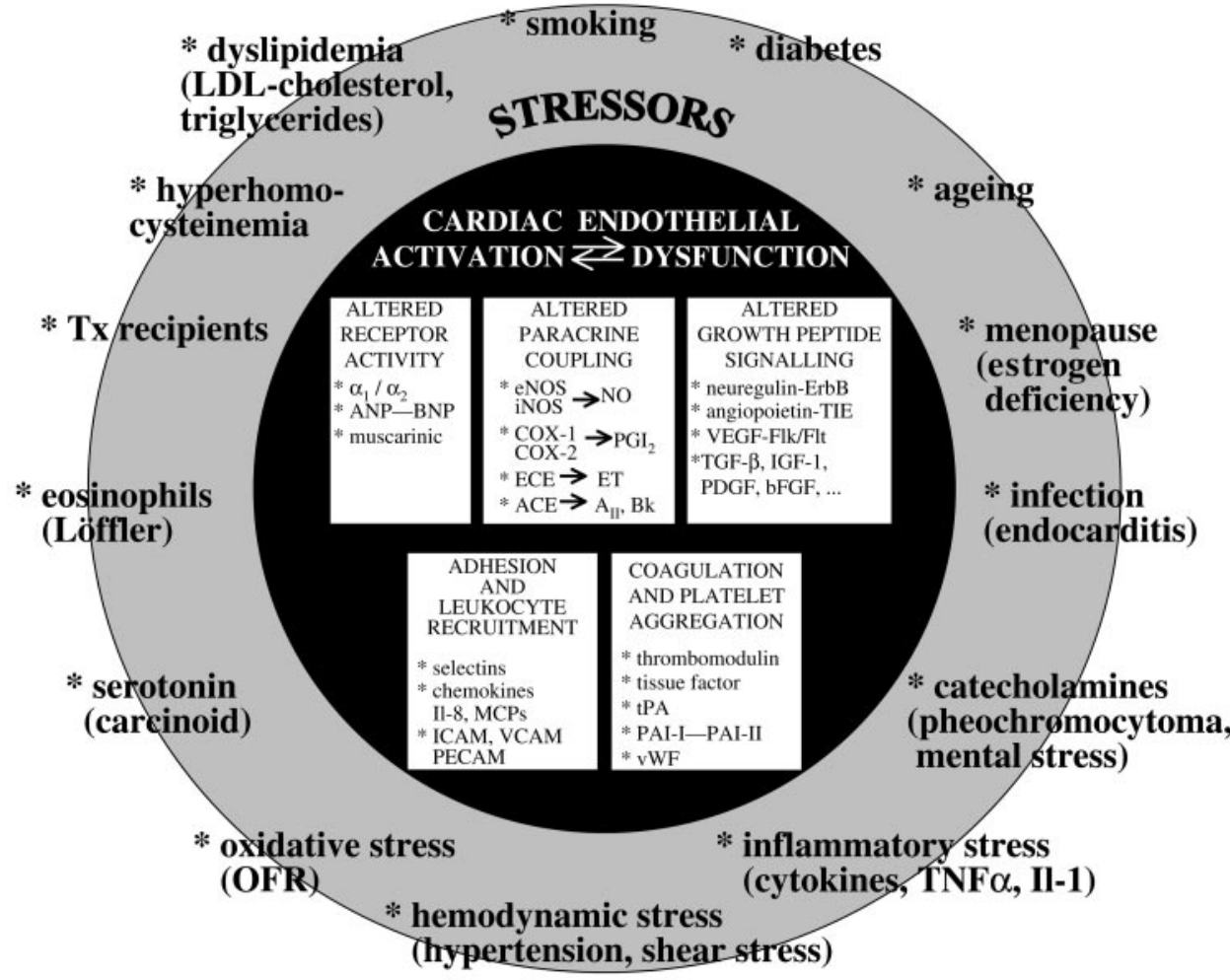

FIG. 10. Cardiac endothelial activation-dysfunction is characterized by a wide spectrum of possible phenotypic changes, inluding, in addition to the wellrecognized changes in auto- and paracrine coupling, altered receptor activity, altered growth peptide signaling, as well as altered (anti)-inflammatory and (anti)coagulant properties. The various diseases and stressors or injuries causing cardiac endothelial activation and subsequent dysfunction are also listed. such as nitroprusside. In addition to a deficient NO pathway, the diagnosis of endothelial dysfunction should, however, also take into account the many other auto-/ paracrine signaling pathways (ECE, COX, ACE) and its (anti-)growth, (anti-)inflammatory, and (anti-)coagulant properties. Experimental studies have indicated that various environmental, endothelial stressors, such as inflammatory stress (cytokines), oxidative stress, and hypoxia, induce alterations of the endothelial phenotype far beyond a mere imbalance of the traditionally described paracrine signalings.

\section{B. Peripheral Vascular Endothelial Dysfunction in Cardiac Failure}

Experimental and clinical observations have shown that endothelial cell activation followed by endothelial dysfunction in cardiac failure is widespread. In both conductive and resistance vessels, as for example in skeletal muscle, endothelial dysfunction has been invoked to explain early fatigue and exercise intolerance in cardiac failure; inappropriate, endothelium-mediated, vasoconstrictor responses with reduced vasodilatory capacity were thought to contribute to the elevated peripheral vascular resistance (126, 249, 261, 291, 312, 427). Peripheral endothelial dysfunction was shown to be an early finding in the progression of cardiac failure $(27,410)$ and ascribed to reduced gene expression of ecNOS and COX-1
(542). Endothelial dysfunction in cardiac failure has also been observed in renal, in mesenteric, and in pulmonary vasculature $(128,131,272,427,491)$. Perhaps most deleterious are the changes in the pulmonary vascular endothelium, because these may lead to impaired clearance of blood-borne constituents and deterioration of overall cardiovascular regulation (see sect. v).

Accordingly, there is ample experimental and clinical evidence that peripheral vascular endothelial dysfunction characterizes and contributes to the syndrome of cardiac failure (161) (Fig. 11). But what about coronary and cardiac endothelial dysfunction in cardiac failure?

\section{Coronary Endothelial Dysfunction in Cardiac Failure}

The suggestion of coronary endothelial dysfunction in cardiac failure followed the demonstration of microspasm in the coronary circulation of Syrian hamsters with dilated cardiomyopathy and of mice infected with Chagas disease (147, 148). Later studies in various experimental animal models $(267,275,424,612)$ as well as in patients with cardiac failure provided direct evidence of endothelial dysfunction in conductive and resistance coronary arteries. In particular, coronary circulation in these patients displayed impaired dilatory responses to acetylcholine or to bradykinin $(168,237,413,587)$. Coronary endothelial dysfunction was thus attributed to decreased 


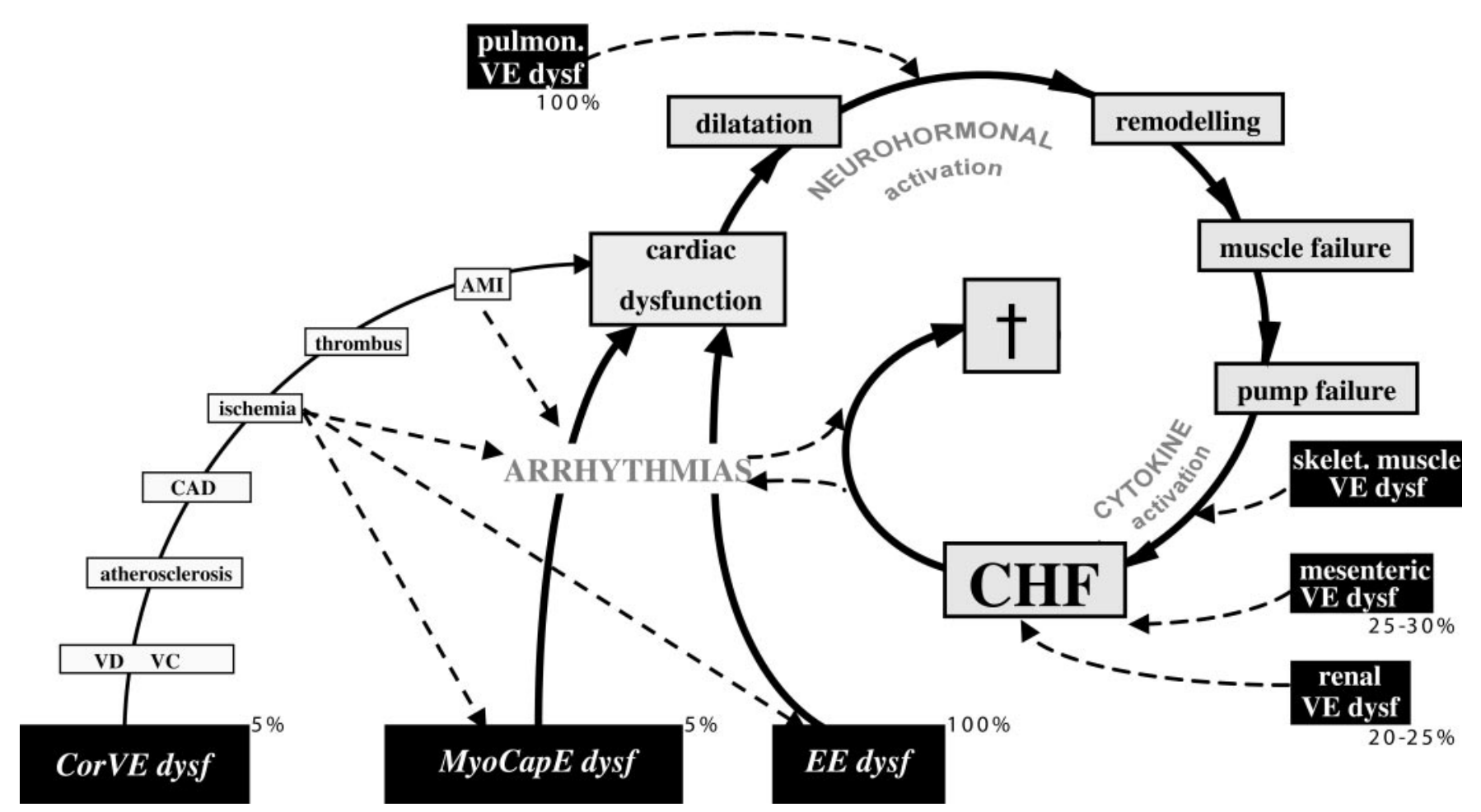

FIG. 11. Role of endothelial dysfunction in the progression of chronic cardiac failure. The diagram depicts the four major underlying and mutually interacting pathophysiological mechanisms. 1) Hemodynamic abnormality includes cardiac dysfunction and overloading (increased pre- and afterload) with cardiac dilatation (Starling) and remodeling (hypertrophy), cardiac muscle (decreased myocardial contractility), and pump failure leading to congestive heart failure (CHF) and death. 2) Neurohormonal imbalance includes increased plasma levels of catecholamines, renin-angiotensinaldosterone, antidiuretic hormone, atrial and brain natriuretic peptide, prostaglandins, and dopamine. 3) Cytokine activation includes overexpression and detrimental effects of various cytokines, as, e.g., tumor necrosis factor- $\alpha$ (TNF- $\alpha$ ) and interleukin-1 and -6 (IL-1, IL-6), on both myocardium and vascular and cardiac endothelium. 4) Endothelial activation-dysfunction-failure involves the entire vascular endothelial system (VE dysf), but most in particular cardiac (EE and MyoCapE) endothelial dysfunction. \%Percentage of cardiac output at rest exposed to VE surface.

coronary endothelial synthesis of NO and seen as an early event in the progression of cardiac dysfunction and failure $(69,362)$. These studies have led to the concept that coronary vascular endothelial dysfunction in patients would trigger inappropriate coronary vasoconstriction, smooth muscle proliferation and remodeling, increased lipid deposition in the vessel wall, and possibly coronary thrombosis; these processes would further accelerate coronary artery disease and by impaired myocardial perfusion indirectly contribute to the progression of cardiac failure and ischemic cardiomyopathy $(124,125,196,342)$. Moreover, impaired myocardial angiogenesis leading to reduced myocardial perfusion and fatal ischemic cardiomyopathy has been observed in mutant mice which lack the vascular endothelial growth factor isoforms $\mathrm{VEGF}_{164}$ and $\mathrm{VEGF}_{188}$ and express only the $\mathrm{VEGF}_{120}$ isoform (72). This suggests a further possibly relevant consequence with impairment of myocardial angiogenesis if the endothelial dysfunction includes also VEGF production.

There has to date, however, been no experimental evidence demonstrating a direct interplay between dysfunctional coronary arterial endothelium and the fail- ing myocardium. The direct involvement of cardiac (EE and MyoCapE) endothelium in modulating myocardial growth, contractile performance, and rhythmicity suggests however that endothelial dysfunction at these two sites could be important in the pathogenesis of cardiac failure $(9,56,58)$.

\section{Cardiac Endothelial Dysfunction in Cardiac Failure}

What is the current evidence that EE and MyoCapE may become dysfunctional and participate in cardiac failure? A number of clinical conditions selectively damage the endocardium and subendocardial interstitial tissue, such as endocarditis, pheochromocytoma, carcinoid syndrome, hypereosinophilic endomyocardial fibrosis (Löffler), endocardial fibroelastosis, and ageing. The role of the $\mathrm{EE}$ in these diseases has only recently been addressed. Typical morphological EE cellular lesions have now been described in conditions of ventricular volume (361) or pressure $(81,541)$ overloading, in experimental diabetes 
and hyperlipidemia (457), as well as in cocaine-induced cardiomyopathy (197). Experimental in vitro studies have demonstrated selective damage of the $\mathrm{EE}$ after exposure to high concentrations of a number of neurohormones and stressors, known to be pathogenetic risk factors in vivo, such as high plasma levels of catecholamines, angiotensin, atrial natriuretic peptide, serotonin, vasopressin, ox-low-density lipoproteins, homocysteine, cholic acid, and eosinophils; these lesions were accompanied by profound changes in the mechanical performance of subjacent myocardium (564). Endothelial damage and dysfunction have also been observed in myocardial capillaries in hypertension, diabetes, hyperlipidemia, and ischemia-reperfusion $(423,462)$. Most cardiovascular risk factors known to be pathogenetic for other vascular endothelial cells (224) appear to affect also EE and MyoCapE as early targets, contributing to the etiology and progression of cardiac failure.

The association of such lesions in EE and MyoCapE with these conditions suggests that they might contribute causally to cardiac failure, but experimental evidence that they do has been missing. In a study in isolated papillary muscles from a model of dilated cardiomyopathy induced by 3 -wk overdrive pacing in dogs, the inotropy doseresponse curve to increasing concentrations of the $\alpha$-agonist phenylephrine showed a significant shift to the higher concentrations, similar to that obtained after selective EE damage of muscles from control animals (324), although no morphological EE damage was seen on scanning electron microscopy. This suggested that EE had become dysfunctional or that desensitization of the EE $\alpha$-receptors had occurred. A similar model in rabbit induced a substantial amount of F-actin stress fibers in EE cells with many myofibroblast-like cells in the subendocardium $(9,253)$. Desensitization of endothelial $\alpha$-receptors in cardiac failure was demonstrated in MyoCapE cells isolated from biopsies of patients with various forms of cardiomyopathy with reduced NO production in response to acetylcholine, bradykinin, and $\alpha$-agonists (267). MyoCapE from patients with dilated idiopathic or ischemic cardiomyopathy displayed a distinct phenotypic shift in endothelial antigen expression for PAL-E, the functional implications of which remain uncertain, although it adds evidence to the concept that changes in MyoCapE occur in chronic cardiac failure, despite lack of other signs of MyoCapE activation, such as expression of adhesion molecules VCAM and E-selectin or of the TGF- $\beta$ binding protein endoglin (358). In another study, immune activation by major histocompatibility complex expression of MyoCapE was demonstrated in a substantial proportion of patients with idiopathic dilated cardiomyopathy, suggesting inflammatory changes (294).

Cardiac endothelial dysfunction is, similarly as coronary vascular endothelial dysfunction, probably an early event in the progression toward cardiac failure. In mod- erate pressure-overload left ventricular hypertrophy in guinea pigs, for example, the typical expected endothelium-dependent NO-induced earlier onset of ventricular relaxation was totally suppressed (344). In this same experimental model, it could, moreover, be demonstrated that coronary endothelial protection from oxidative stress with resulting enhanced NO bioavailability could significantly inhibit the development of left ventricular hypertrophy (36) and restore left ventricular relaxant response to endogenous NO (343a).

More convincing still in favor of a role of cardiac endothelial dysfunction in cardiac failure were experiments in Langendorff-perfused rat hearts 4 wk after coronary ligation (463) (Fig. 12). In this postinfarction model of cardiac failure, the endothelium-mediated increase in coronary flow in response to serotonin was totally suppressed. In papillary muscles removed from these failing hearts, the typical EE-mediated abbreviation of the isometric twitch observed in control muscles in response to selective EE damage was also absent, an effect which could be prevented by pretreatment with the ACE inhibitor captopril. The progression toward cardiac failure and death could be accelerated if the MyoCapE were experimentally damaged by intracoronary low-dose Triton at the time of the coronary ligation (336).

\section{E. Role of NO}

The selective emphasis on NO noted in the recent vascular clinical literature has tended to characterize research also in cardiac research. There have been many studies on the expression of NOS in cardiac tissue from failing hearts. Excessive NO production secondary to the induction of iNOS in failing cardiac tissue would, as in septic shock $(460,592)$, be expected to depress cardiac contraction. This idea has been strengthened further by the observation of high plasma and tissue levels of various cytokines, such as TNF- $\alpha$, known to induce iNOS (213, $257,499,501,584)$. Others however, based on studies in pacing-induced cardiac failure in dogs, have questioned the role of plasma cytokines in the pathogenesis of cardiac failure (474). The major sources of TNF- $\alpha$ production in human idiopathic dilated cardiomyopathy are the cardiomyocytes and cardiac endothelium (538). Increased cardiac tissue levels of TNF- $\alpha$ may directly depress cardiac function and cause dilated cardiomyopathy $(51,293$, 428); the resultant iNOS-dependent coexpression of NO could moreover foster a self-sustaining auto-/paracrine feedback to provoke further TNF- $\alpha$ expression (252).

The observations on NOS expression in cardiac failure have been disappointingly conflicting. Expression of iNOS, for example, is often present in cardiac tissue from failing hearts $(103,127,183,211,221,501,576)$ but has not always been consistently observed (103, 183, 211, 266, 


\section{LANGENDORFF-PERFUSED RAT HEART}
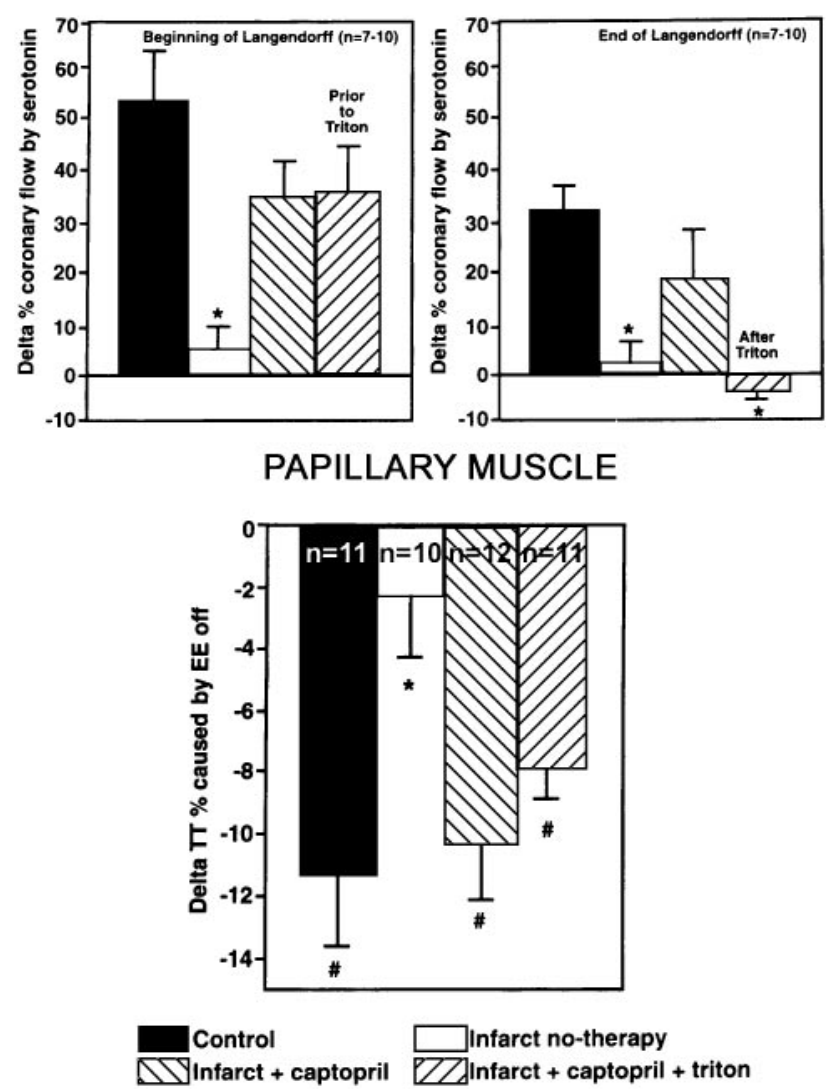

FIG. 12. MyoCapE and EE dysfunction in 4-wk postinfarct cardiac failure in rat. Top left: effects of serotonin-induced (endothelium-dependent) increase in coronary flow in Langendorff-perfused rat heart. Infarct: cardiac failure $4 \mathrm{wk}$ after coronary ligation. Marked increase in coronary flow in hearts from control animals indicates normal endothelium-dependent coronary vasodilatation, which is lost in the untreated postinfarct animals but preserved under treatment with the angiotensinconverting enzyme inhibitor captopril. Top right: same as in left, but in $50 \%$ of the infarct plus captopril group a bolus of Triton X-100 at 1:400 concentration was injected in the aorta just above the coronary arteries; after $15 \mathrm{~min}$, serotonin was delivered and coronary flow measured. Bottom: percent decrease in total tension (TT) due to twitch abbreviation following selective EE removal by a short $0.5 \%$ Triton X-100 exposure in isolated papillary muscle (see also Fig. 1, twitches below). In control muscles with intact EE before EE damage, decreased TT amounts to $\sim 11 \%$. The significant fall in this value in the muscles from postinfarct animals indicates that $\mathrm{EE}$ had already become dysfunctional or damaged before the experimental EE removal with Triton X-100. Preservation of a functional EE under captopril was not abolished in the hearts that in addition had received an intracoronary bolus of diluted Triton X-100. [Modified from Qi et al. (463).]

552, 576). The findings differed depending on the etiology (dilated idiopathic, ischemic, hypertrophic) of the cardiac failure or on the iNOS measuring technique (mRNA, protein, immunohistochemistry). Equally conflicting has been the evidence relating to ecNOS in failing cardiac tissue. In end-stage failing human hearts, ecNOS protein expression was increased in subendocardial cardiomyocytes but decreased in the MyoCapE (183). ecNOS mRNA was decreased in one study (127) but increased in cardio- myocytes in another study (552). The differential expression of ecNOS in normal hearts and the potential for its upregulation in cardiomyocytes has been discussed above and may account for these apparently conflicting findings.

Meanwhile, the role of NO and of ecNOS/iNOS expression in cardiac tissue from various forms of cardiac failure remains controversial $(103,127,183,211,221,265$, $266,501,552,576)$.

Also unclear is the precise role on cardiac function, regardless of the cardiac cellular source, i.e., endothelial cells or cardiomyocytes or macrophages, of the above alterations in cardiac NO production. Intracoronary administration of $N^{\mathrm{G}}$-monomethyl-L-arginine (L-NMMA) in patients with various degrees of ventricular dysfunction had no overall effect on ventricular contractile function (217). Similarly, Drexler et al. (127) reported that L-NMMA had no effect on contractile performance of cardiac muscle isolated from human hearts with end-stage cardiac failure. Paulus and colleagues $(227,444,648)$ found a significant, inverse relationship between ventricular stiffness and iNOS and ecNOS expression in endomyocardial biopsies from patients with dilated cardiomyopathy and a strong positive correlation of endocardial and subendocardial iNOS and ecNOS expression with ventricular contractile performance, suggesting a beneficial effect of endothelium-derived NO in these patients (Fig. 8) (443). Endogenous NO was also shown to be cardioprotective in patients with idiopathic dilated cardiomyopathy by sparing myocardial oxygen consumption through attenuation of the contractile response to $\beta$-adrenergic stimulation (533).

Contrary to these protective actions, the very high NO concentrations resulting from the cytokine-induced overexpression of cardiac iNOS in sepsis-induced myocardial dysfunction (460) and virus-associated (29) cardiomyopathy have, commonly, been invoked as the major mediator of myocardial depression. The putative detrimental effects of these very high iNOS-induced NO concentrations are further supported by the observation that mutant iNOS-deficient mice are protected against endotoxin-induced myocardial dysfunction (592). Mice lacking iNOS have, moreover, improved left ventricular contractile performance, reduced apoptotic cell death, and reduced mortality rate late after myocardial infarction (494). Transgenic mice overexpressing iNOS, on the other hand, did not develop cardiac failure (222).

An acute, endoxin-induced septic model of cardiac failure in rabbit was shown by immunostaining to exhibit transient coinduction of iNOS and inducible $\mathrm{COX}\left(\mathrm{COX}_{2}\right)$ in $\mathrm{EE}$ and endothelium of coronary arterioles, peaking at $\sim 10-12 \mathrm{~h}$, with normalization after $36 \mathrm{~h}$ (370). Simultaneously, the baseline supportive actions of endogenous cardiac ET and $\mathrm{PGI}_{2}$ on myocardial inotropism were transiently accentuated, and the inotropic response to exogenous ET-1 was also significantly enhanced during this 
transient phase. These observations present another example of an adaptive state of cardiac endothelial activation, illustrating the need always to consider all interacting signaling pathways simultaneously.

\section{F. Role of ET}

Plasma ET-1 levels are raised in heart failure, although the significance of these findings was initially questioned because the plasma levels were much lower than those used in pharmacological studies $(106,108)$. The emphasis thus switched to its abluminal secretion and receptor binding properties, plasma ET-1 levels being regarded as luminal spillover, impaired clearance, or augmented ET release in the pulmonary circulation (131, 272, $550,551)$. Levels of ET-1 are enhanced also in EE and MyoCapE as well as in cardiomyocytes of failing hearts. In septicemia and cardiac failure, endothelial cells and macrophages, rather than cardiomyocytes, appeared to be the principal sites of cardiac ET-1 synthesis (182). Marijianowski et al. (358) also found no ET expression in cardiomyocytes from patients with chronic cardiac failure. ppET-1 and ET-1 mRNA and protein levels were markedly upregulated however in cardiomyocytes from infarction-induced failing rat hearts (277). Myocardial interstitial ET-1 levels were decreased in pacing-induced cardiac failure in pigs, despite increased total myocardial tissue ET-1 content, suggesting increased ET-1 uptake, perhaps by cardiomyocytes, in failing hearts (143). ECE-1 mRNA and activity were significantly upregulated however in isolated cardiomyocytes from failing hearts, suggesting activation of a local ET-1 system at the level of the cardiomyocyte (143). Upregulation of a local ET-1 system, with increased mRNA for ppET-1, ECE-1 and $\mathrm{ET}_{\mathrm{A}}$ and $\mathrm{ET}_{\mathrm{B}}$ receptors, was demonstrated also in cardiomyocytes from patients with ischemic but not idiopathic dilated cardiomyopathy, notwithstanding a similar increase in plasma ET-1 in the two groups (518). In another study on end-stage chronic cardiac failure in patients with dilated cardiomyopathy, no significant changes in mRNA expression for ppET-1, ECE-1, and $\mathrm{ET}_{\mathrm{A}}$ receptors were observed compared with control hearts (657); the increased tissue ET-1 concentrations in the latter study, along with downregulation of $\mathrm{ET}_{\mathrm{B}}$ receptor mRNA, would suggest diminished ET-1 clearance rather than increased myocardial ET-1 synthesis.

Activation of the ET-1 pathway might be considered to be adaptive initially $(241,492)$, although soon becoming maladaptive (241), with suggestions that excessive activation of the $\mathrm{ET}_{\mathrm{A}}$ receptors on the cardiomyocytes could lead to direct toxic effects, inappropriate hypertrophy, exhaustion of contractile reserves by chronic inotropic stimulation, and ventricular arrhythmias. It was recently suggested, however, that ET-1 may rather act as a protective factor in blocking either $\beta$-adrenergic agonistinduced (15) or oxidant stress-induced (251) apoptosis in cardiomyocytes via its $\mathrm{ET}_{\mathrm{A}}$ receptor pathway involving multiple downstream signalings, such as, e.g., the calcineurin pathway. Moreover, ET-1 was demonstrated to have a unique oxygen-saving effect by increasing cardiac contractile efficiency (570). In addition, potential antiarrhythmic properties of ET are discussed above (see sect. III) $(244,426,627)$.

\section{G. Role of Other Myocardial-Endothelial Signaling Pathways}

The conflicting findings relating to iNOS and ecNOS expression or functional role in cardiac failure may, as has been discussed above, be related to arbitrary comparison of different animal or human models or of different etiologies of cardiac failure, to tissue sampling taken at different stages during the progression of the disease, to the uncertainty of the cellular source (cardiomyocyte, endothelial cell, macrophage), or to the use of different immunohistochemical or molecular biological measuring techniques $(445,524)$. More important perhaps is the near absence in most of these studies of a systematic analysis of the other endothelial phenotypic changes characteristic of endothelial activation or dysfunction (Fig. 10), which include not only ET and NO, but also $\mathrm{PGI}_{2}$, ANG II, atrial natriuretic peptide, aldosterone, and the more recently discovered NRG, VEGF, and angiopoietin signaling pathways. It was recently reported, for example, that both mRNA and protein levels of $\mathrm{ErbB}_{2}$ and $\mathrm{ErbB}_{4}$ receptors were downregulated at an early stage of cardiac failure in adult animals with chronic hypertrophy secondary to aortic stenosis (481, 639). Knock-out of NRG-1 in this same aortic stenosis animal model did not suppress myocardial hypertrophy, but it did accelerate the development of cardiac failure (638). These observations suggest a role for impaired NRG-ErbB receptor signaling pathway in the transition from compensatory hypertrophy to failure. This view has received indirect support from clinical observations in patients with breast cancer treated with a monoclonal antibody against $\mathrm{ErbB}_{2}$, trastuzumab (Herceptin). A high incidence of severe trastuzumab-induced cardiotoxic cardiomyopathy was observed (152). Mice with conditional ventricle-restricted knock-out of the $\mathrm{ErbB}_{2}$ receptor survive into adulthood but display a rapid onset of dilated cardiomyopathy (78). Whereas the large portion of these animals die prematurely, the survivors display a significant reduction in left ventricular ejection fraction, increased end-diastolic dimensions, myofiber disarray, and upregulation of myocardial atrial naturetic peptide and brain natriuretic peptide $(97,429)$. In cultured cardiomyocytes, trastuzumab caused severe cardiomyopathiclike changes, including disorganization of the cardiomyo- 
cyte cytoskeleton, effects which were blocked or reversed by the addition of NRG (507). Whereas ErbB $_{2}$ overexpression has been associated with the development of neoplastic transformation, $\operatorname{ErbB}_{2}$ receptors and the NRG-ErbB ${ }_{2}$ pathway thus seem at the same time to be cardioprotective. How and to what extent the NRG-ErbB signaling may become maladaptive and contribute to the progression of cardiac failure is still unclear $(78,79)$. Similarly, surviving $5-\mathrm{HT}_{2 \mathrm{~B}}$ receptor-deficient mice exhibit cardiomyopathy with severe vertricular dysfunction and no hypertrophy, perhaps through interaction with the ErbB $_{2}$ pathway (412).

Finally, following hypoxia-reoxygenation in adult rats, the VEGF-Flt-1/Flk-1 and the angiopoietin 1/2-Tie1/2 pathways are significantly upregulated and could, together, constitute a potential basis not only for chronic adaptive angiogenesis, but also for myocardial remodeling.

Accordingly, these findings support the idea of cardiac endothelial activation as an adaptive response often followed by dysfunction at the early stages of cardiac failure. All major cardiovascular risk factors, including hyperlipidemia, hypertension, smoking, oxidative stress, and diabetes, could contribute to these early events. Endothelial auto- and paracrine signaling pathways and most other endothelial activation processes are intimately interconnected and interdependent so that activation or disturbance of any will necessarily affect the others leading to a disturbance of the many cardiac endotheliummyocardial interactions and their normal balance, with a detrimental overall functional outcome that underlies the development and progression of heart failure. The many conflicting data in recent literature on the role of endothelial dysfunction in cardiac failure have reemphasized the need always to consider all interacting signaling pathways simultaneously.

\section{THE ENDOTHELIAL SYSTEM: CONJECTURAL ROLE OF CARDIAC ENDOTHELIUM}

Over the past two decades, physiological sciences have witnessed the emergence of the unified concept of an endothelial system. Despite a number of vascular bedspecific heterogeneities, the endothelium can indeed be viewed as one contiguous structural and functional tissue entity within the body. It is, therefore, tempting to view the endothelium as an autonomous, though well integrated, system within the cardiovascular system. Based on this conjecture, the endothelial system can be seen to act in parallel with and closely linked to other systems: the endocrine system, the immune system, and the central nervous system. All are also well integrated within the cardiovascular system and are all similarly and in concert involved in maintaining overall internal homeostasis of the body. Of all systems, the endothelial system appears to be perhaps the least unstable. Because of its unique position at the interface between on the one hand the individual organs containing part of the central nervous system, and on the other hand the circulating blood carrying most of the endocrine and immune system, the endothelial system is indeed well placed to fulfill a central integrating and stabilizing role for the other homeostatic processes. In a remarkable and provocative review, Ameen (4) has proposed, within a unified concept of the endothelial system, that the highest level of processing and stabilizing control resides in the vascular endothelium of the pulmonary circulation through which all the circulating blood necessarily passes. He postulated that the latter endothelium together with the EE would constitute the central vascular endothelium (CVE) with the pulmonary vascular endothelium acting as the central controlling device. We would add that the EE could be comparably important as a (differential) input-output sensor and feedback device coupled to the CVE.

This proposed CVE represents the largest endothelial surface in the body. It provides the largest single source of endothelial enzymes and contains the highest tissue concentration of endothelial mediators. It receives all circulating blood at least once every 2 min at rest, and more frequently during exercise. The pulmonary vascular endothelium is an important site for biosynthesis as well as clearance of numerous blood-borne molecules, while the EE probably contributes less insofar as exposure is briefer. The CVE is the only site of the endothelial system where the overall composition of the blood can be altered, adjusted, or normalized quickly and efficiently. The list of blood-borne substances, hormones, and mediators, whose concentration is continuously adjusted by the CVE, is a long one, including serotonin, norepinephrine, ET, ANG I, bradykinin, substance P, enkephalins, and various biogenic amines. These adjustments can be conveyed and distributed to all components of the organoriented peripheral vascular endothelium for further adjustments and consumption commensurate to local, organ-specific requirements. ${ }^{13}$ To illustrate this with just

${ }^{13}$ In medicine today, most research effort (and money) is still directed toward studying relatively small and individual aspects of the endothelial system. The endothelium is not commonly thought of as a system at all; rather, it is considered according to a specific clinical organ-oriented specialty, such as cardiology, pneumology, neurology, or nephrology. In cardiology, most attention has for obvious clinical and therapeutic incentives been directed to the coronary vascular endothelium in vasomotor control, and to the pathogenesis and management of coronary atherosclerosis and myocardial ischemia. Yet this arterial endothelial surface comprises only a minute fraction of all endothelial cells in the heart. On the other hand, the MyoCapE and the EE, which directly overlie the cardiomyocytes, account for the largest number of endothelial cells in the heart. The same is true of most other organs where the role of the capillary endothelial cells has long been underestimated, except for the interactions with astrocytes and neurons in the brain (75, 585). In particular, the reciprocal interactions with adjacent organ target cells, such as hepatocytes and Kupffer cells in the liver (14), tubular and 
one example, the pulmonary vascular endothelium is a major site for both ET-1 secretion and extraction; under physiological conditions, ET-1 is balanced across the pulmonary circulation $(131,132,316,609,621)$. The pulmonary vascular endothelium also releases a substantial amount of endogenous big ET, which after conversion to ET-1 may act as a remote modulator of coronary vasomotricity and myocardial performance $(550,551)$.

We have seen above how EE and MyoCapE share many common features and functions that result directly from reciprocal interactions with the subjacent cardiomyocytes. Which of these two endothelial cell types is the more important for the heart is from a physiological point of view irrelevant. Both cell types, depending on the context, are equally indispensable for normal cardiac structure and function. From the point of view of a unified endothelial system, however, EE and MyoCapE are not identical. They distinctly differ with respect to their position and contribution within the endothelial system, the major difference residing in the way in which and the extent to which they perceive and transmit signals.

EE may be considered as functioning at two hierarchic levels of decision making: 1) as a sort of (differential) sensor device which receives all the circulating blood entering and leaving the pulmonary vasculature and 2) as an autocrine or paracrine organ-oriented modulator of cardiac contractile performance, rhythmicity, and growth, the relative importance of these probably being (right vs. left) ventricle specific. ${ }^{14}$ In contrast, MyoCapE functions only as an autocrine or paracrine organ-oriented modulator of cardiac contractile performance, rhythmicity, and growth. Whereas the immense EE surface is continuously exposed to all of the circulating blood, MyoCapE receives $<5 \%$ of cardiac output but controls, at least in the left ventricular wall, the larger portion of myocardial muscle mass.

EE would thus seem well suited to act as a sensor system, for the total circulating amount (concentration or

glomerular epithelial cells in the kidney (31), alveolar epithelial cells in the lungs, or myofiber cells in skeletal muscle are likely to be important but have scarcely been studied.

${ }^{14}$ In some animal species, such as the cod family (Gadidae), the endocardial endothelium has evolved to display a third level of decision making within the endothelial system. Through a well-developed scavenger-receptor-mediated endocytosis, the cod EE cells are most effective in clearing and degrading macromolecular waste products of both physiological and pathophysiological processes from the blood (546). The large EE surface area and the centralized location of these cells are well suited for effective blood surveillance and clearance of harmful endogenous and foreign macromolecules. This scavenging role of EE in the cod is akin to the highly specialized scavenger endothelial cells found in other animals species and at strategic locations in the cardiocirculatory sytem, such as the liver of mammals (540) and the kidney in salmonids $(99,199)$. The capacity of endocardial endothelial cells in mammals to oxidize low-density lipoprotein (399) could be a remnant of this ancestral scavenger function. partial pressure times cavitary flow) of various humoral factors would be more relevant than their concentration or partial pressure alone. A sensor function of the EE would be consistent with its morphological and electrochemical, syncytium-like features. After activation of even a single EE cell, second messengers traverse the abundant gap junctions, to activate neighboring EE cells and amplify their sensor capacity. The lack of gap junctions in MyoCapE would suggest a more local regulatory function of these cells. MyoCapE, at least in the left ventricular wall, will however be exposed to the larger fraction of subjacent cardiomyocytes, and the concentration gradient or partial pressure difference of the circulating stimuli would be more relevant in light of optimal diffusion and for local endothelial modulation of the subjacent myocardium.

EE has been shown to respond to many plasmaborne constituents, as indeed other endothelial cells within the endothelial system. The EE however is distinguished by the higher sensitivity of its responses and by its key localization within the endothelial system at the entrance and exit of the pulmonary circulation. This further endorses the concept of the EE as the distinct sensor device of the CVE. Plasma-borne constituents, to which EE has been shown to be responsive, include metabolites $\left(\mathrm{O}_{2}, \mathrm{CO}_{2}, \mathrm{pH}\right.$, temperature $)(63,525,526)$, hemodynamic signals (flow, volume, pressure) $(280,417,418,563,649)$, eosinophils (521), short bursts of oxygen free radicals (107), platelets (527, 539), biopeptides (acetylcholine, bradykinin, substance $\mathrm{P}$, atrial natriuretic peptide) $(205,268$, $379,391,393,447,543$ ), hormones (ANG II, $\alpha$-adrenoceptor agonists, serotonin, vasopressin, estrogen, aldosterone) $(324,338,377,378,476,509,520)$, ET (326), and cholic acid (91). ${ }^{15}$

The mechanisms involved in the sensor role by the EE might differ for the various constituents. Some of these agents may act through specific EE receptor signaling pathways, e.g., atrial natriuretic peptide receptors (268, 488, 623), mineralocorticoid receptors (338), $\mathrm{ET}_{\mathrm{A}}$ and $\mathrm{ET}_{\mathrm{B}}$ receptors $(395,622)$, angiotensin receptors and ACE (633), as well as receptors for phenylephrine (378), serotonin (520), and vasopressin (509). Other constituents could trigger the EE sensor function through the EE-myocardial auto- and paracrine signaling pathways or

\footnotetext{
${ }^{15}$ Most of these constituents have been investigated in vitro with isolated papillary muscle preparations under rigorous control of temperature, $\mathrm{pH}, \mathrm{PCO}_{2}$, and $\mathrm{PO}_{2}$ in standard Krebs-Ringer solution, sometimes enriched with essential amino acids or small quantities of unspecified serum, as a convenient and highly sensitive, experimental tool to detect EE-mediated modulation of mechanical performance (563), but from which extrapolation to clinical conditions remains uncertain. For some of these constituents, such as atrial natriuretic peptide or short bursts of oxygen free radicals, the presence of an intact EE was obligatory for the papillary muscles to become responsive to them. As for the response to most other ones, the striking feature of an intact EE was to modulate the performance of subjacent myocardium.
} 
by altering the EE BHB properties. Others still, e.g., some of the cellular constituents, could be sensed via adhesion molecules $(6,59)$.

Potentially relevant to the concept of a CVE control system with its sensor role for the EE are any differences between right and left ventricular myocardium and EE $(141,268,484,577,620)$. As previously discussed, prostacyclin $\left(\mathrm{PGI}_{2}\right)$ release is much greater from left than from right ventricular EE under conditions of increased transmural pressure (418).

Other differences between adult left and right ventricular EE have not been sought in any systematic way, and those that have been observed have not been identified with any apparent physiological significance. For example, the $B m x$ tyrosine kinase gene (bone marrow tyrosine kinase gene in chromosome $\mathrm{X}$ ) was found to be specifically expressed in the EE of the left ventricular cavity and in vascular endothelium of large arteries and not in right ventricular EE or to any appreciable extent in its coronary arteries (141). Bmx could be involved in relaying intracavitary hemodynamic signals from the left ventricular EE, which could lead to longterm adjustments of gene expression. It may also participate in the signaling cascade of NO-induced cardioprotection (608). An opposite differential ventricular distribution was observed for cardiac natriuretic peptide receptors with the most abundant receptor expression and binding sites in right ventricular EE, compared with their low expression in left ventricular $\mathrm{EE}$ and in myocardium of both ventricles (268). Interestingly, in right ventricular hypertrophy induced by experimental pulmonary hypertension, binding of natriuretic peptide to right ventricular EE almost disappeared. These observations on the preferential sensing and effector role (379) of right ventricular EE for atrial natriuretic peptide as well as loss of these functions in hypertrophy or mild cardiac failure clearly further endorse and extend the concept of the EE as a sensor device. More information on right-to-left ventricular differences will in the near future become available as we will learn more about left-right asymmetry during cardiac development $(349,645)$.

EE may thus act as a central, differential sensor device within a unified endothelial system. Sensor devices are an essential part of the numerous physiological control systems that regulate homeostasis of the body. From an engineering point of view, control systems in the normal body act by a process of negative feedback and comprise a minimum set of essential components. Their role is to keep some physiological, controlled variable at an almost constant value. Any disturbance that causes the variable to deviate from this target, or set, value must be gauged by a specific sensor device that detects the difference of the variable from its set point. The controlled variable can then be

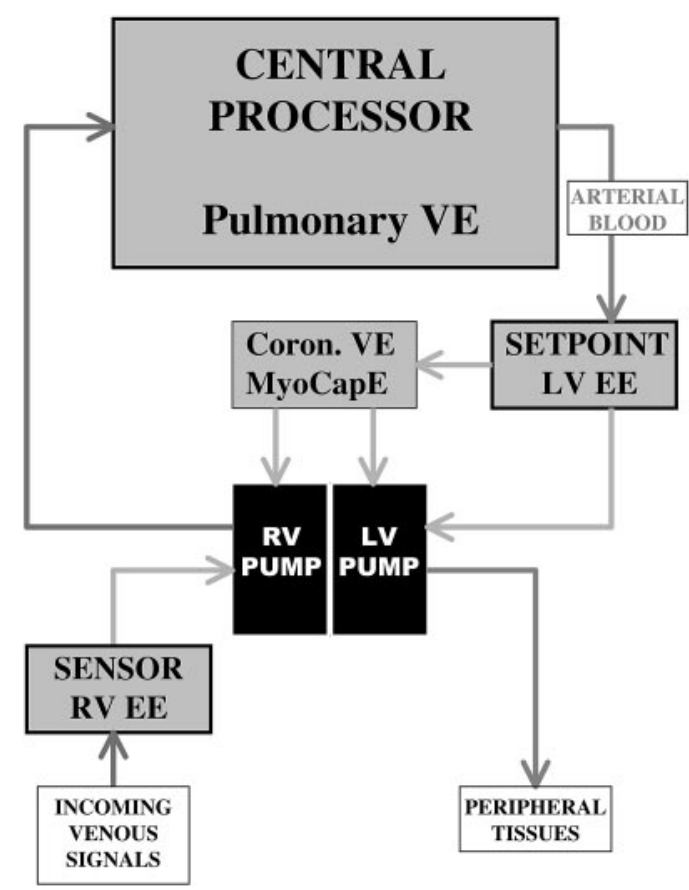

FIG. 13. The endothelial system. Central vascular endothelium with pulmonary vascular endothelium (VE) acting as the central processor and the endocardial endothelium as differential sensor, set point, and feedback devices of the endothelial system. The endocardial-mediated intracavitary autoregulation should be viewed to modulate cardiac performance in parallel with other autoregulatory processes in the heart, such as, e.g., hetero-(Starling) and homeometric autoregulation of the myocardium.

readjusted, or "normalized," by a central controlling processor, connected to the adjustable set-point sensor, from which feedback adjustment can be conveyed to the processor. This feedback loop is the sine qua non of regulation.

In the present context, it is proposed that the controlled variable is the composition of the incoming mixed venous blood while the target value is the composition of the arterial blood in the left ventricle. The sensor device for the mixed venous blood may be considered to be the right ventricular EE, with abluminal signaling to the right ventricular myocardium by which the blood is pumped to the central processor, the pulmonary endothelium. The left ventricular EE sensor would act in a differential way with the right ventricular EE to establish the set point of the control system, from which, through the coronary circulation and MyoCapE, feedback adjustments are provided to the central processor via modulation of the right ventricular pump and pulmonary blood flow (Fig. 13). Short-term control may be mediated by auto- and paracrine mechanisms as well as by the BHB, whereas long-term regulation is mediated through changes in gene expression resulting from the many endothelial-myocardial signaling path- 
ways. ${ }^{16}$ Concept building is far more complex, however, from an integrated physiological point of view, as one can merely speculate about the above axiomatic sequence of events. Experimental confirmation of this unavoidably speculative conceptual model and quantification of the many component mechanisms involved in its integrative physiology will be a complex exercise task. As we will learn more about the cardiovascular endothelium as an endothelial system, perhaps more appropriate models based on complex adaptive system analysis and derived from the growing science of "complexity" may become applicable.

Several arguments can be advanced in support of the consistency of the above conjectural model. For example, because the EE surface area in the right ventricular cavity is many times greater than the EE surface in the left ventricle, its potential sensor power should greatly exceed that of the EE in the left ventricle. It is generally believed that ventricular inner surface trabeculations during embryonic development disappear as a distinct, contractile component of the adult ventricular compartment, but these trabeculations, particularly in the right ventricle, could have acquired the new functional role of enlarging overal EE surface, so optimizing the contribution of the $\mathrm{EE}$ as a sensor device within the endothelial system. The EE cell-to-cardiomyocyte number ratio in the right ventricle, however, is astonishingly high, implying that a substantial proportion of the cardiomyocytes in the right ventricle will be directly accessible to EE-myocardial interaction and able thus to respond to right ventricular EE activation. One can estimate that at least $25 \%$ of the right ventricular cardiomyocytes are under the direct control of the EE; the remaining $75 \%$ will comprise the right ventricular compact myocardium, where they are under the control of the coronary perfusion and the MyoCapE. The substantial potential influence of the EE on right ventricular contractile performance may at least partly explain why considerably fewer cases of right ventricular dysfunction result from occlusion of the right coronary artery than there are of left ventricular dysfunction resulting from occlusion of the left coronary artery, and, why right ventricular function can improve remarkably after right ventricular infarction even in the absence

\footnotetext{
${ }^{16}$ Second-order sensor devices, such as the renal and hepatosplanchnic capillary endothelium, may complement overall coordination of the endothelial system. These endothelial surfaces are, normally, exposed only to, respectively, some 25 and $30 \%$ of the circulating blood flow (Fig. 11), but their filtration and metabolic capacity would importantly add to central processing by the CVE. If these fail, as in hepatic cirrhosis or intestinal ischemia-reperfusion injury, they could compromise the CVE leading to pulmonary and generalized endothelial dysfunction, and multiple organ failure (94, 188, 192, 335, 482, 589). The cerebral endothelium, although exposed to only $12 \%$ of the circulating blood, is so sited as to be able to influence adjacent astrocytes and neurons through reciprocal signaling and thereby play its part toward integrating other homeostatic control systems in the body.
}

of documented revascularization $(112,113)$. Reperfusion of the right coronary artery will, obviously, enhance further recovery of right ventricular function, even after prolonged ischemia $(49,535)$.

As a consequence, right ventricular pump performance and pulmonary blood flow will be adjusted through the right ventricular $\mathrm{EE}$ commensurate to the incoming blood-borne signals of the mixed venous blood. After processing and adjustment by the pulmonary vascular endothelium, the finally normalized blood composition reaches the left ventricular $\mathrm{EE}$, as well as the coronary vascular endothelium, and the MyoCapE in both left and right ventricular wall. Unlike right ventricular $\mathrm{EE}$, left ventricular EE, given the negligible EE cell-to-cardiomyocyte number ratio, would hardly affect left ventricular contractile performance; it could however optimize pump performance through synchronizing its modulatory actions on the terminal Purkinje fiber network and subendocardial neural plexus. The much smaller EE surface area in the left ventricle together with the coronary vascular endothelium and the MyoCapE would then act as the adjustable set point and final feedback regulator of the differential sensor. It would reset and finely tune overall cardiac pump performance by virtue of its indirect coronary vascular-mediated control of the left ventricular pump, while at the same time readjusting, through negative feedback, pulmonary blood flow by virtue of its coronary vascular-mediated control on the compact myocardial fraction of the right ventricle. The ultimate goal would be to optimize delivery of the adjusted, i.e., normalized and stabilized, blood-borne constituents of the arterial blood to the peripheral organs commensurate to overall body needs. As an extension of the adjustable set point of the left ventricular EE and of the vascular endothelium in the coronary conductive and resistance vessels, the large MyoCapE surface could at any time overrule the effects of any other control mechanism on cardiac performance.

Although numerous questions remain unanswered, this does not prevent further speculation. After cardiac transplantation, for example, right ventricular failure is often an early and ominous sign of transplant rejection (41, 554). Similarly, right ventricular dysfunction is an independent predictor of the development of cardiac failure and of death in patients after myocardial infarction (658). The underlying mechanisms remain poorly understood, but endothelial dysfunction of the immense right ventricular EE is likely to impair its sensor and effector function, thus severely perturbing control by the CVE. It could be the pivotal role of the CVE that underlies the often dramatic progression of acute respiratory distress syndrome (ARDS) into a pan-endothelial disorder with fatal multiorgan failure $(188,192,630)$. In cardiac failure too, pulmonary endothelial dysfunction as an early event (128) may lead to pulmonary hypertension and perturba- 
tion of the central processing function of the pulmonary endothelium with diminished corrective control of bloodborne constituents. Pulmonary endothelial failure would then be seen as an ominous development in the progression of cardiac failure.

\section{SUMMARY}

As in our previous review in this journal on integrated cardiac muscle-pump function during relaxation and diastole (64), we try to take a clinical physiological view of global function of the heart. We have of necessity focused first on specific reciprocal signaling interactions of the endocardial (EE) and myocardial capillary endothelial cells (MyoCapE) with cardiomyocytes. Only then could we extrapolate to their interactive roles in the whole cardiac organ within the circulatory system.

We have sampled converging evidence to present and support the conjecture that cardiac endothelial-myocardial interactions play a central and indispensable role in global cardiac organ growth, contractile performance, and rhythmicity. We scan the already large list of reciprocal signaling pathways which determine these interactions in a precisely orchestrated order during embryonic cardiac development and note how many of these are still involved in determining structure and function of the adult heart, pathways which include many traditional autacoids (e.g., NO, ET, prostacyclin, angiotensin) as well as more recently discovered growth peptide molecules with their respective, mostly tyrosine kinase receptors (e.g., VEGF-Flk1/Flt1, NRG-ErbB2/B3/B4, angiopoietin-TIE2/ TEK). More molecular, cardiac endothelial-myocardial signaling pathways remain to be discovered. A still higher scale of complexity may be reached at the $\mathrm{EE}$ where an active BHB may be operative in parallel. This overview can at best be but a staging post in our developing understanding. It is nevertheless already clear that these signaling processes are intimately interconnected and interdependent. The explosively rapid recent growth in our knowledge of gene products and their individual contribution to molecular mechanisms must now give access to their interpretation and to exploring their interaction in concert to that end. Consideration of component pathways in isolation, as has often been the case in studies on NO, cannot provide adequate foundation for understanding their interactive role in the integrated architecture of the whole.

In biomedical sciences, the incentive for the development of new physiological concepts tends prognostically to be led by the priority of healthcare and its industries. Such may partly explain the explosive research efforts over the past 20 years on endothelial function and dysfunction related to coronary artery vasomotricity and atherosclerosis; at the same time, it also explains why the role of cardiac endothelial-myocardial interactions has been largely underestimated for so many years. We suggest in this review that time has come to embrace a new paradigm of structure and function of the heart as a pluricellular organ, in which endothelial cells in particular play an indispensable role. Cardiac endothelial cells, like other vascular endothelial cells, become activated and undergo important phenotypic changes in response to stressors, as exemplified by the familiar risk factors. Cardiac endothelial activation is not always reversible and may in some cases lead to cardiac endothelial dysfunction imbalancing the many endothelial-myocardial signalings. Some of these stressors can activate also the expression and function in cardiomyocytes of physiologically dormant signaling molecules, such as ecNOS mRNA, iNOS mRNA, ET mRNA, ECE, and ACE. Redundancy of these signaling molecules in the cardiomyocytes and perhaps in other nonendothelial cardiac cells as well may initially function as an adaptive escape mechanism, allowing compensation for the cardiac endothelial dysfunction, before progression leads to further imbalance and disease including cardiac failure. This vulnerability of cardiac endothelial cells could explain also the surprisingly beneficial effects obtained by intensive secondary prevention in patients with chronic cardiac failure, whatever its initial etiology.

The differences between EE and MyoCapE in relation to their interactions with cardiomyocytes are instructive. The two cardiac endothelial cell types differ in the ways they perceive and transmit signals and in their hierarchic positions and their contributions within a unified endothelial system. They represent a specific example of the well-recognized phenotypic diversity of vascular endothelial cells in general. This diversity is likely to be environmentally mediated, in large part through differential regulation of the endothelial gene expression by signals from adjacent organ cells, which optimize the microvascular endothelial phenotype to match local, organ-specific requirements (484). Such local coupling mechanisms may limit the range of responsiveness of the endothelial cells to sense, integrate, and transduce only the local signals, providing for a finely tuned endotheliummediated, organ-specific control, while still functionally contributing to a common endothelial system by virtue of their position at the blood/tissue interface.

From an integrative physiological point of view, we can see the emergence of a unified concept of the endothelium as a distinct system within the cardiovascular system.

Previously in this journal (64), we anticipated that global organ approach in terms of integrated systems would, for the future generation of physiologists, present a great challenge to energy and creativity, opposing but complementing as it does, the present investigative effort 
toward ever more discrete subsystems. To reproduce our previous closing words

During a solar eclipse it is the passing over of the moon that allows us a rare chance to look at the sun and that creates the immense and spectacular halo of solar light that is the corona. Indeed, were it not for the passing of the moon, it might never be possible to directly view the sun. It is thus that the new era of molecular biology may pass over and even eclipse the disciplines of physiological science. However, in the passing over, the moon leaves only (however bright) a corona of understanding, for it is not itself the sun.

Now, 10 years later, with the rapid advances in developmental cardiology and new molecular techniques such as targeted gene manipulation, we are witnessing the emergence of new model systems. As evident from the present review, these have already provided novel insights into many of the most basic questions relating to global cardiac organ function. More than ever before, will the corona of understanding, created by the fusion, however momentary, of the passing moon with the sun, mandate conceptual coordination to the organ physiologist and guidance to the clinician.

We gratefully acknowledge Prof. Andrew Henderson for his meticulous reading of the text and for his many critical yet constructive comments.

The transmission electron microscopic and confocal laser micrographs were kindly provided by Dr. Luc Andries. We also thank Heidi Simons and Chris Cuypers for excellent secretarial assistance, in particular Heidi Simons for invaluable and skillful help with the preparation of the illustrations.

Address for reprint requests and other correspondence: D. L. Brutsaert, Univ. of Antwerp, Groenenborgerlaan 171, 2,020 Antwerp, Belgium (E-mail: dirk.brutsaert@skynet.be).

\section{REFERENCES}

1. Acierno R, Agnisola C, Venzi R, and Tota B. Performance of the isolated and perfused working heart of the teleost Conger conger: study of the inotropic effect of prostacyclin. J Comp Physiol B Biochem Syst Environ Physiol 160: 365-371, 1990.

2. Aird WC, Edelberg JM, Weiler GH, Simmons WW, Smith TW, AND ROSENBERG RD. Vascular bed-specific expression of an endothelial cell gene is programmed by the tissue microenvironment. $J$ Cell Biol 138: 1117-1124, 1997.

3. Altug S, Uzun O, Demiryurek AT, Cakici I, Abacioglu N, and KanziK I. The role of nitric oxide in digoxin-induced arrhythmias in guineapigs. Pharmacol Toxicol 84: 3-8, 1999.

4. AmeEn AR. On the physiology of metazoa. Experientia 52: 189-211, 1996.

5. Anagnostou A, Liu Z, Steiner M, Chin K, Lee ES, Kessimian N, And NoGUCHI CT. Erythropoietin receptor mRNA expression in human endothelial cells. Proc Natl Acad Sci USA 91: 3974-3978, 1994.

6. ANDRIES LJ. Endocardial Endothelium: Functional Morphology. Austin, TX: Landes, 1994, p. 1-143.

7. ANDRIES LJ. Functional morphology. In: Endocardial Endothelium, edited by Sys SU and Brutsaert DL. Austin, TX: Landes, 1995, p. 7-39.

8. ANDries LJ, Brutsaert DL, AND Sys SU. Nonuniformity of endothe- lial constitutive nitric oxide synthase distribution in cardiac endothelium. Circ Res 82: 195-203, 1998.

9. Andries lu, Kaluza G, De Keulenaer GW, Mebazaa A, Brutsaert DL, AND Sys SU. Endocardial endothelial dysfunction and heart failure. J Cardiac Failure 2: S195-S202, 1996.

10. Andries LJ, Meulemans AL, AND Brutsaert DL. Ultrasound as a novel method for selective damage of endocardial endothelium. Am J Physiol Heart Circ Physiol 261: H1636-H1642, 1991.

11. Anning PB, Grocott-Mason RM, Lewis MJ, and Shah AM. Enhancement of left ventricular relaxation in the isolated heart by an angiotensin-converting enzyme inhibitor. Circulation 92: 26602665,1995

12. AnVERSA P ANd KaJstura J. Ventricular myocytes are not terminally differentiated in the adult mammalian heart. Circ Res 83: 1-14, 1998.

13. Anversa P, Olinetti G, Melissari M, and Loud AV. Stereological measurement of cellular and subcellular hypertrophy and hyperplasia in the papillary muscle of adult rat. J Mol Cell Cardiol 12: 781-795, 1980.

14. Arai M, Peng XX, Currin RT, Thurman RG, and Lemasters JJ. Protection of sinusoidal endothelial cells against storage/reperfusion injury by prostaglandin $\mathrm{E}_{2}$ derived from Kupffer cells. Transplantation 68: 440-445, 1999.

15. Araki M, Hasegawa K, Iwai Ke, Fujtta M, Sawamura T, Kakita T, Wada H, Morimoto T, and Sasayama S. Endothelin-1 as a protective factor against beta-adrenergic agonist-induced apoptosis in cardiac myocytes. J Am Coll Cardiol 36: 1411-1418, 2000.

16. Au TL, Collins GA, Harvie CJ, and Walker MJ. The actions of prostaglandins $\mathrm{I}_{2}$ and $\mathrm{E}_{2}$ on arrhythmias produced by coronary occlusion in the rat and dog. Prostaglandins 18: 707-720, 1979.

17. Babaei S, Teichert KK, Monge JC, Mohamed F, Bendeck MP, and STEWART DJ. Role of nitric oxide in the angiogenic response in vitro to basic fibroblast growth factor. Circ Res 82: 1007-1015, 1998.

18. BAKER KM AND SINGER HA. Identification and characterization of guinea pig angiotensin II ventricular and atrial receptors: coupling to inositol phosphate production. Circ Res 62: 896-904, 1988.

19. BALDWIN HS AND ARTMAN M. Recent advances in cardiovascular development: promise for the future. Cardiovasc Res 40: 456-468, 1998.

20. Baliga RR, Pimental DR, Zhao YY, Simmons WW, Marchionni MA, SAWYER DB, AND KELLY RA. NRG-1-induced cardiomyocyte hypertrophy. Role of PI-3-kinase, p70(S6K), and MEK-MAPK-RSK. Am J Physiol Heart Circ Physiol 277: H2026-H2037, 1999.

21. BALLERMANN BJ. Endothelial cell activation (clinical conference). Kidney Int 53: 1810-1826, 1998.

22. BALLIGAND JL. Regulation of cardiac beta-adrenergic response by nitric oxide. Cardiovasc Res 43: 607-620, 1999.

23. Balligand JL and CANNon PJ. Nitric oxide synthases and cardiac muscle. Autocrine and paracrine influences. Arterioscler Thromb Vasc Biol 17: 1846-1858, 1997.

24. Balligand Jl, Kelly RA, Marsden PA, Smith TW, and Michel T. Control of cardiac muscle cell function by an endogenous nitric oxide signaling system. Proc Natl Acad Sci USA 90: 347-351, 1993.

25. Balligand JL, Kobzik L, Han X, Kaye DM, Belhassen L, O'Hara DS, Kelly RA, SMith TW, AND Michel T. Nitric oxide-dependent parasympathetic signaling is due to activation of constitutive endothelial (type III) nitric oxide synthase in cardiac myocytes. $J$ Biol Chem 270: 14582-14586, 1995.

26. Banai S, Shweiki D, Pinson A, Chandra M, Lazarovici G, and Keshet E. Upregulation of vascular endothelial growth factor expression induced by myocardial ischaemia: implications for coronary angiogenesis. Cardiovasc Res 28: 1176-1179, 1994.

27. BANK AJ, LEe PC, AND KuBo SH. Endothelial dysfunction in patients with heart failure: relationship to disease severity. J Cardiac Failure 6: 29-36, 2000.

28. Bannon PG, Kim MJ, Dean RT, and Dawes J. Augmentation of vascular endothelial barrier function by heparin and low molecular weight heparin. Thromb Haemostasis 73: 706-712, 1995.

29. Barbaro G, Di Lorenzo G, Soldini M, Giancaspro G, Grisorio B, Pellicelli A, AND BARBARINI G. Intensity of myocardial expression of inducible nitric oxide synthase influences the clinical course of human immunodeficiency virus-associated cardiomyopathy. 
Gruppo Italiano per lo Studio Cardiologico dei pazienti affetti da AIDS (GISCA). Circulation 100: 933-939, 1999.

30. Bartram U, Molin DGM, Wisse LJ, Mohamad A, Sanford LP, Doetschman T, Speer CP, Poelmann RE, and Gittenberger-DeGROOT AC. Double-outlet right ventricle and overriding tricuspid valve reflect disturbances of looping, myocardialization, endocardial cushion differentiation, and apoptosis in TGF- $\beta 2$-knockout mice. Circulation 103: 2745-2752, 2001.

31. Bassenge E. Endothelial function in different organs. Prog Cardiovasc Dis 39: 209-228, 1996.

32. Bassenge E and Heusch G. Endothelial and neuro-humoral control of coronary blood flow in health and disease. Rev Physiol Biochem Pharmacol 116: 77-165, 1990.

33. BAVIK C, WARD SJ, AND CHAMBON P. Developmental abnormalities in cultured mouse embryos deprived of retinoic by inhibition of yolksac retinol binding protein synthesis. Proc Natl Acad Sci USA 93: 3110-3114, 1996.

34. Bayraktutan U, YANG ZK, AND ShaH AM. Selective dysregulation of nitric oxide synthase type 3 in cardiac myocytes but not coronary microvascular endothelial cells of spontaneously hypertensive rat. Cardiovasc Res 38: 719-726, 1998.

35. Becker R, Merkely B, Bauer A, Geller L, Fazekas L, Freigang KD, Voss F, Senges JC, Kuebler W, ANd Schoels W. Ventricular arrhythmias induced by endothelin-1 or by acute ischemia: a comparative analysis using three-dimensional mapping. Cardiovasc Res 45: $310-320,2000$.

36. Bell JP, Mosfer SI, Lang D, Donaldson F, and Lewis MJ. Vitamin C and quinapril abrogate LVH and endothelial dysfunction in aorticbanded guinea pigs. Am J Physiol Heart Circ Physiol 281: H1704H1710, 2001

37. Beltrami AP, Urbanek K, Kajstura J, Yan SM, Finato N, Bussani R, Nadal-Ginard B, Silvestri F, Leri A, Beltrami CA, and Anversa P. Evidence that human cardiac myocytes divide after myocardial infarction. N Engl J Med 344: 1750-1757, 2001.

38. Bernstein RD, OchoA FY, Xu X, Forfia P, Shen W, Thompson CI, ANd HINTZE TH. Function and production of nitric oxide in the coronary circulation of the conscious dog during exercise. Circ Res 79: 840-848, 1996.

39. BÉNY JL. Information networks in the arterial wall. NIPS 14: 68-73, 1999.

40. Bi W, Drake CJ, and Schwarz JJ. The transcription factor MEF2Cnull mouse exhibits complex vascular malformations and reduced cardiac expression of angiopoietin 1 and VEGF. Dev Biol 211: 255-267, 1999

41. Birks EJ, Owen VJ, Burton PBJ, Bishop AE, Banner NR, Khaghan A, PolaK JM, AND YACOUB MH. Tumor necrosis factor- $\alpha$ is expressed in donor heart and predicts right ventricular failure after human heart transplantation. Circulation 102: 326-331, 2000.

42. Bleyl SB, Mumford BR, Brown-Harrison MC, Pagotto LT, Carey JC, PYSHER TJ, WARD K, AND CHIN TK. Xq28-linked noncompaction of the left ventricular myocardium: prenatal diagnosis and pathologic analysis of affected individuals. Am J Med Genet 72: 257-265, 1997.

43. Bloch W, Fleischmann BK, Lorke DE, Andressen C, Hops B, HesCHELER J, AND ADDICKS K. Nitric oxide synthase expression and role during cardiomyogenesis. Cardiovasc Res 43: 675-684, 1999.

44. Borchard U, HAFNER D, AND KoJdA G. Endogenous nitric oxide production accelerates diastolic depolarization in the guinea-pig sinoatrial node (Abstract). Circulation 100: I-121, 1999.

45. Bouloumie A, SchinI KV, AND Busse R. Vascular endothelial growth factor up-regulates nitric oxide synthase expression in endothelial cells. Cardiovasc Res 41: 773-780, 1999.

46. Bouman HG, Broekhuizen ML, BaAsten AM, GitTenberger-DE-Groot AC, AND WENINK AC. Diminished growth of atrioventricular cushion tissue in stage 24 retinoic acid-treated chicken embryos. Dev Dyn 213: 50-58, 1998.

47. Bouman HGA, Broekhuizen MLA, BaAsten AMJ, GitTenberger-DeGROOT AC, AND WeninK ACG. Stereological study of stage 34 chicken hearts with looping disturbances after retinoic acid treatment: disturbed growth of myocardium and atrioventricular cushion tissue. Anat Rec 248: 242-250, 1997.

48. Bourreau JP, Banijamali HS, and Challice CE. Modification of excitation-contraction coupling in cat ventricular myocardium fol- lowing endocardial damage. Can J Physiol Pharmacol 71: 254-262, 1993

49. Bowers TR, O'Neill WW, Grines C, Pica MC, Safian RD, and GoldSTEIN JA. Effect of reperfusion on biventricular function and survival after right ventricular infarction. $N$ Engl J Med 338: 933-940, 1998.

50. Boyer AS, Ayerinskas II, Vincent EB, McKinney LA, Weeks DL, AND RUNYAN RB. TGFbeta2 and TGFbeta3 have separate and sequential activities during epithelial-mesenchymal cell transformation in the embryonic heart. Dev Biol 208: 530-545, 1999.

51. Bozkurt B, Kribis SB, Clubb FJJ, Michael LH, Didenko VV, Hornsby PJ, Seta Y, Oral H, Sinale FG, and Mann DL. Pathophysiologically relevant concentrations of tumor necrosis factor-alpha promote progressive left ventricular dysfunction and remodeling in rats. Circulation 97: 1382-1391, 1998.

52. Brady AJ, Warren JB, Poole-Wilson PA, Williams TJ, and Harding SE. Nitric oxide attenuates cardiac myocyte contraction. Am J Physiol Heart Circ Physiol 265: H176-H182, 1993.

53. BRandt R, NowaK J, and Sonnenfeld T. Prostaglandin formation from exogenous precursor in homogenates of human cardiac tissue. Basic Res Cardiol 79: 135-141, 1984.

54. Brown CB, Boyer AS, Runyan RB, ANd BarnetT JV. Requirement of type III TGF-beta receptor for endocardial cell transformation in the heart. Science 283: 2080-2082, 1999.

54a.Brunner F, Andrew P, Wolkart G, Zechner R, and Mayer B. Myocardial contractile function and heart rate in mice with myocytespecific overexpression of endothelial nitric oxide synthase. Circulation 104: 3097-3102, 2001.

55. Brutsaert DL. The endocardium. Annu Rev Physiol 51: 263-273, 1989.

56. BRUTSAERT DL. Role of endocardium in cardiac overloading and failure. Eur Heart J 11 Suppl G: 8-16, 1990.

57. BRUTSAERT DL. Endocardial and coronary endothelial control of cardiac performance. NIPS 8: 82-86, 1993.

58. Brutsaert DL AND ANDRIEs LJ. The endocardial endothelium. Am J Physiol Heart Circ Physiol 263: H985-H1002, 1992.

59. Brutsaert DL, De Keulenaer GW, Fransen P, Mohan P, Kaluza GL, ANdries LJ, Rouleau JL, and SYs SU. The cardiac endothelium: functional morphology, development, and physiology. Prog Cardiovasc Dis 39: 239-262, 1996.

60. Brutsaert DL, Fransen P, Andries LJ, De Keulenaer GW, and Sys SU. Cardiac endothelium and myocardial function. Cardiovasc Res 38: 281-290, 1998.

61. Brutsaert DL and Meulemans AL. Transendothelial ionic exchange underlies endocardial control of myocardial performance $(\mathrm{Ab}$ stract). Biophys $J$ 53: 59a, 1988.

62. Brutsaert DL, Meulemans AL, Sipido KR, and Sys SU. The endocardium modulates the performance of myocardium (Louvain, Nov. 1986) (Abstract). Arch Int Physiol Biochem 95: 4, 1987.

63. Brutsaert DL, Meulemans AL, Sipido KR, and Sys SU. Effects of damaging the endocardial surface on the mechanical performance of isolated cardiac muscle. Circ Res 62: 358-366, 1988.

64. BRUTSAERT DL AND Sys SU. Relaxation and diastole of the heart. Physiol Rev 69: 1228-1315, 1989.

65. BRUTSAeRt DL AND Sys SU. Diastolic dysfunction in heart failure. J Cardiac Failure 3: 225-242, 1997.

66. BundGAARD M. The three-dimensional organization of tight junctions in a capillary endothelium revealed by serial-section electron microscopy. J Ultrastruct Res 88: 1-17, 1984.

67. Calaghan SC AND White E. Contribution of angiotensin II, endothelin 1 and the endothelium to the slow inotropic response to stretch in ferret papillary muscle. Pfügers Arch 441: 514-520, 2001.

68. Calderone A, Thaik CM, Takahashi N, Chang DLF, and Colucci WS. Nitric oxide, atrial natriuretic peptide, and cyclic GMP inhibit the growth-promoting effects of norepinephrine in cardiac myocytes and fibroblasts. J Clin Invest 101: 812-818, 1998.

69. Cannan CR, McGoon MD, Holmes DRJ, and Lerman A. Altered coronary endothelial function in a patient with asymptomatic left ventricular dysfunction. Int J Cardiol 53: 147-151, 1996.

70. CARMeliet P AND Collen D. Role of vascular endothelial growth factor and vascular endothelial growth factor receptors in vascular development. Curr Top Microbiol Immunol 237: 133-158, 1999.

71. Carmeliet P, Ferreira V, Breier G, Pollefeyt S, Kieckens L, Gert- 
Senstein M, Fahrig M, Vandenhoeck A, Harpal K, Eberhardt C, Declercq C, Pawling J, Moons L, Collen D, Risau W, and Nagy A. Abnormal blood vessel development and lethality in embryos lacking a single VEGF allele. Nature 380: 435-439, 1996.

72. Carmeliet P, NG YS, Nuyens D, Theilmeier G, Brusselmans K, Cornelissen I, Ehler E, Kakkar VV, Stalmans I, Mattot V, Perriard JC, Dewerchin M, Flameng W, Nagy A, Lupu F, Moons L, Collen D, D'AMore PA, AND SHIMA DT. Impaired myocardial angiogenesis and ischemic cardiomyopathy in mice lacking the vascular endothelial growth factor isoforms VEGF164 and VEGF188. Nat Med 5: 495502, 1999.

73. Carmona R, Gonzalez IM, Macias D, Perez-Pomares JM, Garcia GL, AND MunOz CR. Immunolocalization of the transcription factor Slug in the developing avian heart. Anat Embryol Berl 201: 103-109, 2000.

74. CARTER TD AND PEARSOn JD. Regulation of prostacyclin synthesis in endothelial cells. NIPS 7: 64-69, 1992

75. CeBallos G and RuBio R. Coculture of astroglial and vascular endothelial cells as apposing layers enhances the transcellular transport of hypoxanthine. J Neurochem 64: 991-999, 1995.

76. Chesnais JM, Fischmeister R, AND Mery PF. Peroxynitrite is a positive inotropic agent in atrial and ventricular fibres of the frog heart. J Physiol 521: 375-388, 1999.

77. Chesnais JM, Fischmeister R, And Mery PF. Positive and negative inotropic effects of $\mathrm{NO}$ donors in atrial and ventricular fibres of the frog heart. J Physiol 518: 449-461, 1999.

78. CHIEN KR. Stress pathways and heart failure. Cell 98: 555-558, 1999.

79. ChiEn KR. Genomic circuits and the integrative biology of cardiac diseases. Nature 407: 227-232, 2000.

80. Chin TK, Perloff JK, Williams RG, Jue K, and Mohrmann R. Isolated noncompaction of left ventricular myocardium. A study of eight cases. Circulation 82: 507-513, 1990.

81. ChU GX, Ling Q, AND Guo ZG. Effects of endocardial endothelium in myocardial mechanics of hypertrophied myocardium of rats. Acta Pharmacol Sinica 16: 352-356, 1995.

82. Chu GX, Zhang Y, AND Guo ZG. Endocardial endothelium modulates cardiac responses to histamine and impromidine in isolated working right ventricle of guinea pigs. Chung Kuo Yao Li Hsueh Pao 14: 225-228, 1993.

83. Chua BH, Chua CC, Diglio CA, AND Siu BB. Regulation of endothelin-1 mRNA by angiotensin II in rat heart endothelial cells. Biochim Biophys Acta 1178: 201-206, 1993.

84. Chua CC, Hamdy RC, AND Chua BH. Upregulation of vascular endothelial growth factor by $\mathrm{H}_{2} \mathrm{O}_{2}$ in rat heart endothelial cells. Free Radical Biol Med 25: 891-897, 1998.

85. Chuck ET, Freeman DM, Watanabe M, and Rosenbaum DS. Changing activation sequence in the embryonic chick heart. Implications for the development of the His-Purkinje system. Circ Res 81: 470476, 1997.

86. Cleeter MW, Cooper JM, Darley U, Moncada S, and Schapira AH. Reversible inhibition of cytochrome $c$ oxidase, the terminal enzyme of the mitochondrial respiratory chain, by nitric oxide. Implications for neurodegenerative diseases. FEBS Lett 345: 50-54, 1994.

87. Clouthier DE, Hosoda K, Richardson JA, Williams SC, Yanagisawa H, Kuwaki T, Kumada M, Hammer RE, and Yanagisawa M. Cranial and cardiac neural crest defects in endothelin-A receptor-deficient mice. Development 125: 813-824, 1998.

88. Cohen GL AND Mikawa T. The fate diversity of mesodermal cells within the heart field during chicken early embryogenesis. Dev Biol 177: 265-273, 1996.

89. CoKer SJ AND McGrath MT. Can captopril modify experimentally induced cardiac arrhythmias? (Abstract). J Mol Cell Cardiol 17: 41, 1985.

90. Coker SJ, Parratt JR, Ledingham IM, and Zeitlin IJ. Thromboxane and prostacyclin release from ischaemic myocardium in relation to arrhythmias. Nature 291: 323-324, 1981.

91. Colpaert CG, Vandenbroucke MP, Andries LJ, Van Marck EA, and BRUTSAERT DL. Role of endocardial endothelium in the positive inotropic effect of cholic acid in isolated myocardium. J Cardiovasc Pharmacol 20 Suppl 12: S179-S182, 1992.

91a.Condorelli G, Borello U, De Angelis L, Latronico MSD, Coletta M, Galli R, Balconi G, Follenzi A, Frati G, Cusella De Angelis MG, Gioglio L, Amuchastegui S, Adorini L, Naldini L, Vescovi A, Dejana
E, AND Cossu G. Cardiomyocytes induce endothelial cells to transdifferentiate into cardiac muscle: implications for myocardium regeneration. Proc Natl Acad Sci USA 98: 10733-10738, 2001.

92. Cotton JM, Kearney MT, MacCarthy PA, Grocott-Mason RM, McCleAN DR, Heymes C, RichaRdSON PJ, AND SHAH AM. Effects of nitric oxide synthase inhibition on basal function and the force-frequency relationship in the normal and failing human heart in vivo. Circulation 104: 2318-2323, 2001.

93. Couttenye MM, De Clerck NM, Herman AG, and Brutsaert DL. Effects of prostacyclin on contractile properties of isolated mammalian cardiac muscle. J Cardiovasc Pharmacol 7: 971-976, 1985.

94. Cremona G, Higenbottam TW, Mayoral V, Alexander G, DeMONCHEAUX E, BoRland C, RoE P, and Jones GJ. Elevated exhaled nitric oxide in patients with hepatopulmonary syndrome. Eur Respir $J$ 8: 1883-1885, 1995.

95. CRICK SJ, ANDERSON RH, Ho SY, AND SHEPPARd MN. Localisation and quantitation of autonomic innervation in the porcine heart. II. Endocardium, myocardium and epicardium. J Anat 195: 359-373, 1999.

96. Crick SJ, Sheppard MN, Ho SY, AND ANDERSON RH. Localisation and quantitation of autonomic innervation in the porcine heart. I. Conduction system. J Anat 195: 341-357, 1999.

97. Czelik C, Garrat an, Britsch S, Osterziel KJ, Dietz R, Chien KR, AND BIRCHMEIER C. Erbb2 signaling and cardiac disease: a new mouse model for dilated cardiomyopathy (Abstract). Circulation 102: 475,2000 .

98. Damron DS and Summers BA. Arachidonic acid enhances contraction and intracellular $\mathrm{Ca}^{2+}$ transients in individual rat ventricular myocytes. Am J Physiol Heart Circ Physiol 272: H350-H359, 1997.

99. Dannevig BH, Lauve A, Press C, and Landsverk T. Receptor-mediated endocytosis and phagocytosis by rainbow trout head kidney sinusoidal cells. Fish Shellfish Immunol 4: 3-18, 1994.

100. Davenport AP, Nunez DJ, Hall JA, Kaumann AJ, and Brown MJ. Autoradiographical localization of binding sites for porcine $\left[{ }^{125} \mathrm{I}\right]$ endothelin-1 in humans, pigs, and rats: functional relevance in humans. J Cardiovasc Pharmacol 13 Suppl 5: S166-S170, 1989.

101. DAVIES PF. Flow-mediated endothelial mechanotransduction. Physiol Rev 75: 519-560, 1995.

102. Davis S And Yancopoulos GD. The angiopoietins: Yin and Yang in angiogenesis. Curr Top Microbiol Immunol 237: 173-185, 1999.

103. De Belder AJ, Radomski MW, Why HJ, Richardson PJ, and Martin JF. Myocardial calcium-independent nitric oxide synthase activity is present in dilated cardiomyopathy, myocarditis, and postpartum cardiomyopathy but not in ischaemic or valvar heart disease. $\mathrm{Br}$ Heart $J$ 74: 426-430, 1995

104. De Hert SG, Gillebert TC, and Brutsaert DL. Alteration of left ventricular endocardial function by intracavitary high-power ultrasound interacts with volume, inotropic state, and alpha 1-adrenergic stimulation. Circulation 87: 1275-1285, 1993.

105. De Hurtado MC, Alvarez BV, Ennis IL, and Cingolani He. Stimulation of myocardial $\mathrm{Na}^{+}$-independent $\mathrm{Cl}^{-}-\mathrm{HCO}_{3}^{-}$exchanger by angiotensin II is mediated by endogenous endothelin. Circ Res 86: $622-627,2000$.

105a.Dejana E and Del Maschio A. Molecular organization and functional regulation of cell to cell junctions in the endothelium. Thromb Haemostasis 74: 309-312, 1995.

106. De Keulenaer GW. Endothelial Stress Activation. Emphasis on Redox-Based and Adaptive Physiocellular Aspects (PhD thesis). Antwerp, Belgium: Univ. of Antwerp, 1999.

107. De Keulenaer GW, Andries LJ, Sys SU, and Brutsaert DL. Endothelin-mediated positive inotropic effect induced by reactive oxygen species in isolated cardiac muscle. Circ Res 76: 878-884, 1995.

108. De Keulenaer GW and BRutsaert DL. Dilated cardiomyopathy: changing pathophysiological concepts and mechanisms of dysfunction. J Cardiac Surg 14: 64-74, 1999.

109. De Keulenaer GW, Fransen P, Brutsaert DL, and Sys SU. Decreased myocardial contractility after damage to endocardial endothelium is not merely caused by loss of endothelin production. Cardiovasc Res 30: 646-647, 1995.

110. De Keulenaer GW and Sys SU. Endothelial control of myocardial performance. In: Endocardial Endothelium: Control of Cardiac Performance, edited by Sys SU and Brutsaert DL. Austin, TX: Landes, 1995. 
111. De la Pompa Jl, Timmerman la, Takimoto H, Yoshida H, Elia AJ, Samper E, Potter J, Wakeham A, Marengere L, Langille BL, CRABTREE GR, AND MAK TW. Role of the NF-ATc transcription factor in morphogenesis of cardiac valves and septum. Nature 392: 182186, 1998.

112. Dell'Italia LJ. Reperfusion for right ventricular infarction. $N$ Engl J Med 338: 978-980, 1998.

113. Dell'ttalia LJ, Lembo NJ, Starling MR, Crawford MH, Simmons RS, Lasher JC, Blumhardt R, LanCaster J, and O'Rourke RA. Hemodynamically important right ventricular infarction: follow-up evaluation of right ventricular systolic function at rest and during exercise with radionuclide ventriculography and respiratory gas exchange. Circulation 75: 996-1003, 1987.

114. De Mey JG And Vanhoutte PM. Heterogeneous behavior of the canine arterial and venous wall. Importance of the endothelium. Circ Res 51: 439-447, 1982.

115. Dempsey PJ, McCallum ZT, Kent KM, and Cooper T. Direct myocardial effects of angiotensin II. Am J Physiol 220: 477-481, 1971.

116. De Nucci G, Thomas R, D'Orleans JP, Antunes E, Walder C, WARNER TD, AND VANE JR. Pressor effects of circulating endothelin are limited by its removal in the pulmonary circulation and by the release of prostacyclin and endothelium-derived relaxing factor. Proc Natl Acad Sci USA 85: 9797-9800, 1988.

118. Depre C, Vanoverschelde JL, Goudemant JF, Mottet I, and Hue L. Protection against ischemic injury by nonvasoactive concentrations of nitric oxide synthase inhibitors in the perfused rabbit heart. Circulation 92: 1911-1918, 1995.

119. DeRuiter MC, Poelmann Re, VanMunsteren JC, Mironov V, MarkWALD RR, AND GITTENBERGER-DE-GROOT AC. Embryonic endothelial cells transdifferentiate into mesenchymal cells expressing smooth muscle actins in vivo and in vitro. Circ Res 80: 444-451, 1997.

120. Dickson MC, Slager HG, Duffie E, Mummery Cl, ANd AkHurst RJ. RNA and protein localisations of TGF beta 2 in the early mouse embryo suggest an involvement in cardiac development. Development 117: 625-639, 1993.

121. Digicaylioglu M, Bichet S, Marti HH, Wenger RH, Rivas La, Bauer C, AND GASSMANN M. Localization of specific erythropoietin binding sites in defined areas of the mouse brain. Proc Natl Acad Sci USA 92: 3717-3720, 1995.

122. Discigil B, Pearson PJ, Chua YL, Evora PR, Seccombe JF, and SCHAFF HV. Novel technique to bioassay endocardium-derived nitric oxide from the beating heart. Ann Thorac Surg 59: 1182-1186, 1995.

123. Dostal DE and BAKer KM. The cardiac renin-angiotensin system: conceptual, or a regulator of cardiac function? Circ Res 85: 643$650,1999$.

124. Drexler H. Endothelial dysfunction: clinical implications. Prog Cardiovasc Dis 39: 287-324, 1997.

125. Drexter H. Nitric oxide and coronary endothelial dysfunction in humans. Cardiovasc Res 43: 572-579, 1999.

126. Drexler H, Hayoz D, Munzel T, Hornig B, Just H, Brunner HR, And ZELIS R. Endothelial function in chronic congestive heart failure. Am J Cardiol 69: 1596-1601, 1992.

127. Drexler H, Kastner S, Strobel A, Studer R, Brodde OE, and HASENFUSS G. Expression, activity and functional significance of inducible nitric oxide synthase in the failing human heart. $J \mathrm{Am}$ Coll Cardiol 32: 955-963, 1998.

128. Driss AB, Devaux C, Henrion D, Duriez M, Thuillez C, Levy BI, AND Michel JB. Hemodynamic stresses induce endothelial dysfunction and remodeling of pulmonary artery in experimental compensated heart failure. Circulation 101: 2764-2770, 2000.

129. Drysdale TA, Patterson KD, Saha M, and Krieg PA. Retinoic acid can block differentiation of the myocardium after heart specification. Dev Biol 188: 205-215, 1997.

130. Duncker DJ, Stubenttsky R, Tonino PAL, And Verdouw PD. Nitric oxide contributes to the regulation of vasomotor tone but does not modulate $\mathrm{O}_{2}$ consumption in exercising swine. Cardiovasc Res 47: $738-748,2000$.

131. Dupuis J, Rouleau JL, ANd Cernacek P. Reduced pulmonary clearance of endothelin-1 contributes to the increase of circulating levels in heart failure secondary to myocardial infarction. Circulation 98: 1684-1687, 1998.

132. Dupuis J, Stewart DJ, Cernacek P, and Gosselin G. Human pulmo- nary circulation is an important site for both clearance and production of endothelin-1. Circulation 94: 1578-1584, 1996.

133. Dusek J, Ostadal B, and Duskova M. Postnatal persistence of spongy myocardium with embryonic blood supply. Arch Pathol 99: 312-317, 1975.

134. Dyson E, Sucov HM, Kubalak SW, Schmid-Schonbein GW, DeLano FA, Evans RM, Ross J, and ChiEn KR. Atrial-like phenotype is associated with embryonic ventricular failure in retinoid $\mathrm{X}$ receptor alpha -/- mice. Proc Natl Acad Sci USA 92: 7386-7390, 1995.

135. DZAU VJ. Circulating versus local renin-angiotensin system in cardiovascular homeostasis. Circulation 77: 4-13, 1988.

136. Edelberg JM, Aird WC, Wu W, Rayburn H, Mamuya WS, Mercola M, AND ROSENBERG RD. PDGF mediates cardiac microvascular communication. J Clin Invest 102: 837-843, 1998.

137. Eid H, Larson DM, Springhorn JP, Attawia MA, Nayak RC, Smith TW, AND KeLLY RA. Role of epicardial mesothelial cells in the modification of phenotype and function of adult rat ventricular myocytes in primary coculture. Circ Res 71: 40-50, 1992.

138. Eid H, O'NeIll M, Smith TW, and Kelly RA. Plasticity of adult rat cardiac myocytes in long-term culture: role of non-muscle cardiocytes in the maintenance of the adult phenotype in vitro (Abstract). Circulation 82 Suppl III: 689, 1990.

139. EISENBerg CA AND BADER D. QCE-6: a clonal cell line with cardiac myogenic and endothelial cell potentials. Dev Biol 167: 469-481, 1995.

140. Eisenberg LM and Markwald RR. Molecular regulation of atrioventricular valvuloseptal morphogenesis. Circ Res 77: 1-6, 1995.

141. Ekman N, Lymboussaki A, Vastrik I, Sarvas K, Kaipainen A, AND Alitalo K. Bmx tyrosine kinase is specifically expressed in the endocardium and the endothelium of large arteries. Circulation 96: 1729-1732, 1997.

142. Emanueli C, Maestri R, Corradi D, Marchione R, Minasi A, Tozzi MG, Salis MB, Straino S, Capogrossi MC, Olivetti G, and Madeddu P. Dilated and failing cardiomyopathy in bradykinin $\mathrm{B}_{2}$ receptor knockout mice. Circulation 100: 2359-2365, 1999.

143. Ergul A, Walker CA, Goldberg A, Baicu SC, Hendrick JW, King MK, AND SPINALE FG. ET-1 in the myocardial interstitium: relation to myocyte ECE activity and expression. Am J Physiol Heart Circ Physiol Heart Circ Physiol 278: H2050-H2056, 2000.

144. Erickson SL, O'Shea KS, Ghaboosi N, Loverro L, Frantz G, Bauer M, LU LH, AND Moore MW. ErbB3 is required for normal cerebellar and cardiac development: a comparison with ErbB2-and heregulindeficient mice. Development 124: 4999-5011, 1997.

145. ERIKSSON U AND Alitalo K. Structure, expression and receptorbinding properties of novel vascular endothelial growth factors. Curr Top Microbiol Immunol 237: 41-57, 1999.

146. Evans HG, Lewis MJ, AND SHAH AM. Modulation of myocardial relaxation by basal release of endothelin from endocardial endothelium. Cardiovasc Res 28: 1694-1699, 1994.

147. Factor SM, Cho S, Wittner M, and Tanowitz H. Abnormalities of the coronary microcirculation in acute murine Chagas' disease. Am J Trop Med Hyg 34: 246-253, 1985.

148. Factor SM, Minase T, Cho S, Dominitz R, and Sonnenblick EH. Microvascular spasm in the cardiomyopathic Syrian hamster: a preventable cause of focal myocardial necrosis. Circulation 66: 342-354, 1982.

149. FARquHARSON CA AND Struthers AD. Spironolactone increases nitric oxide bioactivity, improves endothelial vasodilator dysfunction, and suppresses vascular angiotensin I/angiotensin II conversion in patients with chronic heart failure. Circulation 101: 594$597,2000$.

150. Fassina G, Tessari F, and Dorigo P. Positive inotropic effect of a stable analog of $\mathrm{PGI}_{2}$ and of $\mathrm{PGI}_{2}$ on isolated guinea pig atria. Mechanism of action. Pharmacol Res Commun 15: 735-749, 1983.

151. Fei L, BARON AD, Henry DP, AND ZIPES DP. Intrapericardial delivery of L-arginine reduces the increased severity of ventricular arrhythmias during sympathetic stimulation in dogs with acute coronary occlusion: nitric oxide modulates sympathetic effects on ventricular electrophysiological properties. Circulation 96: 4044-4049, 1997.

152. Feldman AM, Lorell BH, and Reis SE. Trastuzumab in the treatment of metastatic breast cancer: anticancer therapy versus cardiotoxicity. Circulation 102: 272-274, 2000. 
153. Feletou M and Vanhoutte PM. Endothelium-dependent hyperpolarization of canine coronary smooth muscle. $\mathrm{Br} J$ Pharmacol 93: 515-524, 1988 .

154. Feng Q, Song W, and Lu X. Basal nitric oxide production from endothelial nitric oxide synthase is essential for cardiomyocyte survival (Abstract). Circulation 102: II-240, 2000.

155. Feng Q, Song W, and Lu X. Endothelial nitric oxide synthase deficiency increases apoptosis in embryonic heart and induces congenital septal defects (Abstract). Circulation 102: II-109, 2000.

156. Feron O, Belhassen L, Kobzik L, SMith TW, Kelly RA, and Michel T Endothelial nitric oxide synthase targeting to caveolae. Specific interactions with caveolin isoforms in cardiac myocytes and endothelial cells. J Biol Chem 271: 22810-22814, 1996.

157. Feron O AND Kelly RA. The caveolar paradox: suppressing, inducing, and terminating eNOS signaling. Circ Res 88: 129-131, 2001.

158. FERRARA N. Vascular endothelial growth factor: molecular and biological aspects. Curr Top Microbiol Immunol 237: 1-30, 1999.

159. Ferrara N, Carver MK, Chen H, Dowd M, Lu L, O'Shea KS, Powell BL, Hillan KJ, AND Moore MW. Heterozygous embryonic lethality induced by targeted inactivation of the VEGF gene. Nature 380: $439-442,1996$.

160. Ferrara N, Houck K, Jakeman L, and Leung DW. The biology of vascular endothelial growth factor. Endocr Rev 18: 4-25, 1998.

161. Ferrari R, Bachetti T, Agnoletti L, Comini L, and Curello S. Endothelial function and dysfunction in heart failure. Eur Heart $J$ 19 Suppl G: G41-G47, 1998.

162. Ferrero E, Ferrero ME, Pardi R, and Zocchi MR. The platelet endothelial cell adhesion molecule-1 (PECAM1) contributes to endothelial barrier function. FEBS Lett 374: 323-326, 1995.

163. Fischer TA, Ungureanu LD, Singh K, de Zengotita J, DeUgarte D, Alali A, Gadbut AP, Lee MA, Balligand JL, Kifor I, Smith TW, and KeLLY RA. Regulation of bFGF expression and ANG II secretion in cardiac myocytes and microvascular endothelial cells. Am J Physiol Heart Circ Physiol 272: H958-H968, 1997.

164. Fishman MC AND ChIEN KR. Fashioning the vertebrate heart: earliest embryonic decisions. Development 124: 2099-2117, 1997.

165. Fleming I and Busse R. Signal transduction of eNOS activation. Cardiovasc Res 43: 532-541, 1999.

166. Flesch M, Kilter H, Cremers B, Lenz O, Sudkamp M, Kuhn RF, and Bонм M. Acute effects of nitric oxide and cyclic GMP on human myocardial contractility. J Pharmacol Exp Ther 281: 1340-1349, 1997.

167. Fong GH, Rossant J, Gertsenstein M, and Breitman ML. Role of the Flt-1 receptor tyrosine kinase in regulating the assembly of vascular endothelium. Nature 376: 66-70, 1995.

168. Forstermann U, Mugge A, Alheid U, Bode SM, and Frolich JC. Endothelium-derived relaxing factor (EDRF): a defence mechanism against platelet aggregation and vasospasm in human coronary arteries. Eur Heart J 10 Suppl F: 36-43, 1989.

169. Franco D, Jing Y, Wagenaar GTM, Lamers WH, and Moorman AFM. The trabecular component of the embryonic ventricle. In: The Developing Heart, edited by Ostadal B. Philadelphia, PA: Lippincott-Raven, 1996, 51-60.

170. Franco D, Kelly R, Lamers WH, Buckingham M, and Moorman AF. Regionalized transcriptional domains of myosin light chain $3 \mathrm{f}$ transgenes in the embryonic mouse heart: morphogenetic implications. Dev Biol 188: 17-33, 1997.

171. Franco D, Lamers WH, and Moorman AF. Patterns of expression in the developing myocardium: towards a morphologically integrated transcriptional model. Cardiovasc Res 38: 25-53, 1998.

172. Fransen P, Andries LJ, Van Bedaf D, Demolder M, and Sys SU. Cell coupling in cultured endocardial endothelium (Abstract). Pflügers Arch 431: R323, 1996.

173. Fransen P and Demolder M. Physicochemical properties of the endocardial endothelium. In: Endocardial Endothelium: Control of Cardiac Performance, edited by Sys SU and Brutsaert DL. Austin, TX: Landes, 1995.

174. Fransen P, Hendrickx J, Brutsaert DL, and Sys SU. Distribution and role of $\mathrm{Na}^{+} / \mathrm{K}^{+}$ATPase in endocardial endothelium. Cardiovasc Res 52: 487-499, 2001.

175. Fransen $\mathrm{P}$ and Sys SU. $\mathrm{K}^{+}$and $\mathrm{Cl}^{-}$contribute to resting membrane conductance of cultured porcine endocardial endothelial cells. Am J Physiol Heart Circ Physiol 272: H1770-H1779, 1997.
176. Fransen P, Sys SU, and Brutsaert DL. Endocardial endothelial cell communication. Presented at INABIS' 98. Proc Int World Congr Biomed Sci 5th McMaster Univ Canada 1998. (Available at http:// www.mcmaster.ca/inabis98/nilius/fransen0434/index.html)

177. Fransen PF, Demolder MJ, and Brutsaert DL. Whole cell membrane currents in cultured pig endocardial endothelial cells. Am J Physiol Heart Circ Physiol 268: H2036-H2047, 1995.

178. Freer RJ, Pappano AJ, Peach MJ, Bing KT, McLean MJ, Vogel S, and SPERELAKIS N. Mechanisms for the positive inotropic effect of angiotensin II on isolated cardiac muscle. Circ Res 39: 178-183, 1976.

179. FreLin C AND Guedin D. Why are circulating concentrations of endothelin-1 so low? Cardiovasc Res 28: 1613-1622, 1994.

180. Fujiyama S, Matsubara H, Nozawa Y, Maruyama K, Mori $Y$, Tsutsumi Y, Masaki H, Uchiyama Y, Koyama Y, Nose A, Iba O, Tateishi E, Ogata N, Jyo N, Higashiyama S, and Iwasaka T. Angiotensin AT(1) and AT(2) receptors differentially regulate angiopoietin-2 and vascular endothelial growth factor expression and angiogenesis by modulating heparin binding-epidermal growth factor (EGF)-mediated EGF receptor transactivation. Circ Res 88: 22-29, 2001.

181. Fukazawa R, Ogawa Si, Frantz S, Marchionni MA, Kelly RA, and SAwYER DB. Differential signaling by epidermal growth factor and neuregulin in rat cardiac myocytes and cardiac microvascular endothelial cells (Abstract). Circulation 102: II-138, 2000.

182. FukUCHI M AND GIAID A. Expression of endothelin-1 and endothelinconverting enzyme-1 mRNAs and proteins in failing human hearts. J Cardiovasc Pharmacol 31 Suppl 1: S421-S423, 1998.

183. FukUChI M, Hussain SN, AND GIAID A. Heterogeneous expression and activity of endothelial and inducible nitric oxide synthases in endstage human heart failure: their relation to lesion site and betaadrenergic receptor therapy. Circulation 98: 132-139, 1998.

184. Fulton D, Papapetropoulos A, Zhang X, Catravas JD, Hintze TH, AND SESSA WC. Quantification of eNOS mRNA in the canine cardiac vasculature by competitive PCR. Am J Physiol Heart Circ Physiol 278: H658-H665, 2000.

185. Furchgott RF and Vanhoutte PM. Endothelium-derived relaxing and contracting factors. FASEB $J$ 3: 2007-2018, 1989.

186. FURCHGOTT RF AND ZAWADZKI JV. The obligatory role of endothelial cells in the relaxation of arterial smooth muscle by acetylcholine. Nature 288: 373-376, 1980

187. Ganz P, Davies PF, Leopold JA, Gimbrone MA JR, and Alexander RW. Short- and long-term interactions of endothelium and vascular smooth muscle in coculture: effects on cyclic GMP production. Proc Natl Acad Sci USA 83: 3552-3556, 1986.

188. Garcia JG AND Natarajan V. Signal transduction in pulmonary endothelium. Implications for lung vascular dysfunction. Chest 102: 592-607, 1992.

189. Gassmann M, Casagranda F, Orioli D, Simon H, Lai C, Klein R, and LEMKE G. Aberrant neural and cardiac development in mice lacking the ErbB4 neuregulin receptor. Nature 378: 390-394, 1995.

190. Gattuso A, Mazza R, Pellegrino D, and Tota B. Endocardial endothelium mediates luminal ACh-NO signaling in isolated frog heart. Am J Physiol Heart Circ Physiol 276: H633-H641, 1999.

191. GEORGe EL, BALDwin HS, AND HynEs RO. Fibronectins are essential for heart and blood vessel morphogenesis but are dispensable for initial specification of precursor cells. Blood 90: 3073-3081, 1997.

192. Gerkin TM, Oldham KT, Guice KS, Hinshaw DB, and Ryan US. Intestinal ischemia-reperfusion injury causes pulmonary endothelial cell ATP depletion. Ann Surg 217: 48-56, 1993.

193. GERRITSEN ME. Physiological and pathophysiological roles of eicosanoids in the microcirculation. Cardiovasc Res 32: 720-732, 1996.

194. GERRITSEN ME AND CHELI CD. Arachidonic acid and prostaglandin endoperoxide metabolism in isolated rabbit and coronary microvessels and isolated and cultivated coronary microvessel endothelial cells. J Clin Invest 72: 1658-1671, 1983.

195. GERRITSEn ME AND PRINTZ MP. Sites of prostaglandin synthesis in the bovine heart and isolated bovine coronary microvessels. Circ Res 49: 1152-1163, 1981.

196. Gheorghiade M And Bonow RO. Chronic heart failure in the United States: a manifestation of coronary artery disease. Circulation 97 : 282-289, 1998

197. Gilloteaux J and Dalbec JP. Transplacental cardiotoxicity of cocaine: atrial damage following treatment in early pregnancy. Scanning Microsc 5: 519-529, 1991. 
198. GIMBRone MA JR AND TopPER JN. Biology of the vessel wall: endothelium. In: Molecular Basis of Cardiovascular Disease, edited by Chien KR. Philadelphia, PA; Saunders, 1999, p. 331-348.

199. GJoen T AND Berg T. Metabolism of low density lipoproteins in rainbow trout. Fish Physiol Biochem 9: 453-461, 1992.

200. Gomes PM, Mandarim-De-Lacerda CA, and Dumas HM. Stereology and immunohistochemistry of the myocardium in experimental hypertension: long-term and low-dosage administration of inhibitor of the nitric oxide synthesis. Pathobiology 67: 26-33, 1999.

201. Gourdie RG, Mima T, Thompson RP, and Mikawa T. Terminal diversification of the myocyte lineage generates Purkinje fibers of the cardiac conduction system. Development 121: 1423-1431, 1995.

202. Gourdie RG, Wei Y, Kim D, Klatt SC, and Mikawa T. Endothelininduced conversion of embryonic heart muscle cells into impulseconducting Purkinje fibers. Proc Natl Acad Sci USA 95: 6815-6818, 1998.

203. Grant GA, ABbotT NJ, AND Janigro D. Understanding the physiology of the blood-brain barrier: in vitro models. NIPS 13: 287-293, 1998.

204. Griffith TM, Edwards DH, Lewis MJ, Newby AC, and Henderson $\mathrm{AH}$. The nature of endothelium-derived vascular relaxant factor. Nature 308: 645-647, 1984.

205. Grocott-Mason R, Anning P, Evans H, Lewis MJ, and Shah AM. Modulation of left ventricular relaxation in isolated ejecting heart by endogenous nitric oxide. Am J Physiol Heart Circ Physiol 267: H1804-H1813, 1994.

206. Grocott-Mason R, Fort S, Lewis MJ, and Shah AM. Myocardial relaxant effect of exogenous nitric oxide in isolated ejecting hearts. Am J Physiol Heart Circ Physiol 266: H1699-H1705, 1994.

207. Grocott-Mason R, MacCarthy PA, Muldins N, and Shah AM. Effect of inhibition of nitric oxide synthase on the myocardial forcefrequency relation in normal humans in vivo (Abstract). Circulation 98: I-761, 1998.

208. Gruber PJ, Kubalak SW, Pexieder T, Sucov HM, Evans RM, and CHIEN KR. RXR alpha deficiency confers genetic susceptibility for aortic sac, conotruncal, atrioventricular cushion, and ventricular muscle defects in mice. J Clin Invest 98: 1332-1343, 1996.

209. Guarda E, Katwa LC, Myers PR, Tyagi SC, and Weber KT. Effects of endothelins on collagen turnover in cardiac fibroblasts. Cardiovasc Res 27: 2130-2134, 1993.

210. Guarda E, Myers PR, Brilla CG, Tyagi SC, and Weber KT. Endothelial cell induced modulation of cardiac fibroblast collagen metabolism. Cardiovasc Res 27: 1004-1008, 1993.

211. Gurubhagavatula I, Amrani Y, Pratico D, Ruberg Fl, Albelda SM, AND PANETTIERI RA JR. Engagement of human PECAM-1 (CD31) on human endothelial cells increases intracellular calcium ion concentration and stimulates prostacyclin release. J Clin Invest 101: 212 $222,1998$.

212. Gyurko R, Kuhlencordt P, Fishman MC, and Huang PL. Modulation of mouse cardiac function in vivo by eNOS and ANP. Am J Physiol Heart Circ Physiol 278: H971-H981, 2000.

213. Habib FM, Springall DR, Davies GJ, OAkley CM, Yacoub MH, and POLAK JM. Tumour necrosis factor and inducible nitric oxide synthase in dilated cardiomyopathy. Lancet 347: 1151-1155, 1996.

214. Haigh JJ, Gerber HP, Ferrara N, and Wagner EF. Conditional inactivation of VEGF-A in areas of collagen2a1 expression results in embryonic lethality in the heterozygous state. Development 127 : 1445-1453, 2000.

215. Han Y, Dennis JE, Cohen GL, Bader DM, and Fischman DA. Expression of sarcomeric myosin in the presumptive myocardium of chicken embryos occurs within six hours of myocyte commitment. Dev Dyn 193: 257-265, 1992.

216. Harding P, Carretero OA, And LaPointe MC. Effects of interleukin-1 beta and nitric oxide on cardiac myocytes. Hypertension 25: 421-430, 1995.

217. Hare JM, Loh E, Creager MA, and ColucCi WS. Nitric oxide inhibits the positive inotropic response to beta-adrenergic stimulation in humans with left ventricular dysfunction. Circulation 92: 21982203, 1995

218. Hashimoto E, Ogita T, Nakaoka T, Matsuoka R, Takao A, and Kira Y. Rapid induction of vascular endothelial growth factor expression by transient ischemia in rat heart. Am J Physiol Heart Circ Physiol 267: H1948-H1954, 1994.
219. Hassanabad ZF, Furman BL, Parratt JR, and Aughey E. Coronary endothelial dysfunction increases the severity of ischaemia-induced ventricular arrhythmias in rat isolated perfused hearts. $B a$ sic Res Cardiol 93: 241-249, 1998.

220. Hayakawa H AND RAIJ L. The link among nitric oxide synthase activity, endothelial function, and aortic and ventricular hypertrophy in hypertension. Hypertension 29: 235-241, 1997.

221. Haywood Ga, Tsao PS, der-Leyen HE, Mann MJ, Keeling PJ, Trindade PT, Lewis NP, Byrne CD, Rickenbacher PR, Bishopric NH, CoOKe JP, McKenna WJ, AND Fowler MB. Expression of inducible nitric oxide synthase in human heart failure. Circulation 93: 10871094, 1996.

222. Heger J, Gödecke A, Flögel U, Merx MW, Molojavyi A, KühNVELTEN WN, AND Schrader J. Cardiac-specific overexpression of inducible nitric oxide synthase does not result in severe cardiac dysfunction. Circ Res 90: 93-106, 2002.

223. Heine UI, Roberts AB, Munoz EF, Roche NS, And Sporn MB. Effects of retinoid deficiency on the development of the heart and vascular system of the quail embryo. Virchows Arch B Cell Pathol Incl Mol Pathol 50: 135-152, 1985.

224. Henderson AH. "It all used to be so simple in the old days." A personal view. Eur Heart $J$ 22: 648-653, 2001.

225. Hendrickson RJ, Cappadona C, Yankah EN, Sttzmann JV, Cahill PA, AND REDMOND EM. Sustained pulsatile flow regulates endothelial nitric oxide synthase and cyclooxygenase expression in co-cultured vascular endothelial and smooth muscle cells. $\mathrm{J} \mathrm{Mol} \mathrm{Cell}$ Cardiol 31: 619-629, 1999.

226. Hertig CM, Kubalak SW, Wang Y, and Chien KR. Synergistic roles of neuregulin-1 and insulin-like growth factor-I in activation of the phosphatidylinositol 3-kinase pathway and cardiac chamber morphogenesis. J Biol Chem 274: 37362-37369, 1999.

227. Heymes C, Vanderheyden M, Bronzwaer JG, Shah AM, and Paulus WJ. Endomyocardial nitric oxide synthase and left ventricular preload reserve in dilated cardiomyopathy. Circulation 99: 3009-3016, 1999.

228. HiRakow R. Development of the cardiac blood vessels in staged human embryos. Acta Anat Basel 115: 220-230, 1983.

229. Hirota H, Chen J, Betz UA, Rajewsky K, Gu Y, Ross J, Muller W, AND CHIEN KR. Loss of a gp130 cardiac muscle cell survival pathway is a critical event in the onset of heart failure during biomechanical stress. Cell 97: 189-198, 1999.

230. Hood JD, Meininger CJ, Ziche M, ANd GRanger HJ. VEGF upregulates ecNOS message, protein, and NO production in human endothelial cells. Am J Physiol Heart Circ Physiol 274: H1054-H1058, 1998.

231. Hoyer J, Distler A, HaAse W, And Gogelein H. $\mathrm{Ca}^{2+}$ influx through stretch-activated cation channels activates maxi $\mathrm{K}^{+}$channels in porcine endocardial endothelium. Proc Natl Acad Sci USA 91: 2367-2371, 1994.

232. HSU HY, Nicholson AC, AND HAJJAR DP. Basic fibroblast growth factor-induced low density lipoprotein receptor transcription and surface expression. Signal transduction pathways mediated by the bFGF receptor tyrosine kinase. J Biol Chem 269: 9213-9220, 1994.

233. Ichida F, Tsubata S, Bowles KR, Haneda N, Uese K, Miyawaki T, Dreyer J, Messina J, Li H, Bowles NE, AND Towbin JA. Novel gene mutations in patients with left ventricular noncompaction or Barth syndrome. Circulation 103: 1256-1263, 2001.

234. IgNARRo LJ. Biological actions and properties of endothelium-derived nitric oxide formed and released from artery and vein. Circ Res 65: 1-21, 1989.

235. Ignarro LJ, Byrns RE, Buga GM, and Wood KS. Endotheliumderived relaxing factor from pulmonary artery and vein possesses pharmacologic and chemical properties identical to those of nitric oxide radical. Circ Res 61: 866-879, 1987.

236. Inagami T, NARUSe M, AND Hoover R. Endothelium as an endocrine organ. Annu Rev Physiol 57: 171-189, 1995.

237. Inoue T, Sakai Y, Morooka S, Hayashi T, Takayanagi K, Yamaguchi $\mathrm{H}$, KAKOI H, AND TAKABATAKE Y. Vasodilatory capacity of coronary resistance vessels in dilated cardiomyopathy. Am Heart $J$ 127: 376-381, 1994.

238. Ishigai Y, Mori T, Ikeda T, Fukuzawa A, and Shibano T. Role of bradykinin-NO pathway in prevention of cardiac hypertrophy by 
ACE inhibitor in rat cardiomyocytes. Am J Physiol Heart Circ Physiol 273: H2659-H2663, 1997.

239. Ito H, Hirata Y, Adachi S, Tanaka M, Tsujino M, Koike A, Nogami A, Murumo F, AND Hiroe M. Endothelin-1 is an autocrine/paracrine factor in the mechanism of angiotensin II-induced hypertrophy in cultured rat cardiomyocytes. J Clin Invest 92: 398-403, 1993.

240. Ito H, Hirata Y, Hiroe M, Tsujino M, Adachi S, Takamoto T, NitTa M, TANiguchi K, and Marumo F. Endothelin-1 induces hypertrophy with enhanced expression of muscle-specific genes in cultured neonatal rat cardiomyocytes. Circ Res 69: 209-215, 1991.

241. Iwanaga Y, Kihara Y, Hasegawa K, Inagaki K, Yoneda T, Kaburagi S, ARAKI M, AND SASAYAMa S. Cardiac endothelin-1 plays a critical role in the functional deterioration of left ventricles during the transition from compensatory hypertrophy to congestive heart failure in salt-sensitive hypertensive rats. Circulation 98: 2065-2073, 1998.

242. JaCoBSEN AN, Du XJ, DART AM, AND WoodCock EA. Ins $(1,4,5) \mathrm{P}_{3}$ and arrhythmogenic responses during myocardial reperfusion: evidence for receptor specificity. Am J Physiol Heart Circ Physiol 273: H1119-H1125, 1997.

243. Jacques D, Sader S, Choufani S, D'Orleans JP, and Charest D. Endothelin-1 regulates cytosolic and nuclear $\mathrm{Ca}^{2+}$ in human endocardial endothelium. J Cardiovasc Pharmacol 36: S397-S400, 2000.

244. James AF, Xie LH, Fujitani Y, Hayashi S, and Horie M. Inhibition of the cardiac protein kinase A-dependent chloride conductance by endothelin-1. Nature 370: 297-300, 1994.

245. Jenni R, Goebel N, Tartini R, Schneider J, Arbenz U, and Oelz O. Persisting myocardial sinusoids of both ventricles as an isolated anomaly: echocardiographic, angiographic, and pathologic anatomical findings. Cardiovasc Intervent Radiol 9: 127-131, 1986.

246. JENNI R, ROJAS J, AND OECHSLIN E. Isolated noncompaction of the myocardium. N Engl J Med 340: 966-967, 1999

247. JUGDUTT BI AND KHAN MI. Effect of prolonged nitrate therapy on left ventricular remodeling after canine acute myocardial infarction. Circulation 89: 2297-2307, 1994.

248. Junga G, Kneifel S, Von Smekal A, Steinert H, and Bauersfeld U. Myocardial ischaemia in children with isolated ventricular noncompaction. Eur Heart J 20: 910-916, 1999.

249. KaISER L, SPICKARD RC, and Olivier NB. Heart failure depresses endothelium-dependent responses in canine femoral artery. Am J Physiol Heart Circ Physiol 256: H962-H967, 1989.

250. Kajstura J, Leri A, Finato N, Di loreto C, Beltrami CA, and ANVERSA P. Myocyte proliferation in end-stage cardiac failure in humans. Proc Natl Acad Sci USA 95: 8801-8805, 1998.

251. Kakita T, Hasegawa K, Iwai KE, Adachi S, Morimoto T, Wada H, Kawamura T, Yanazume T, and Sasayama S. Calcineurin pathway is required for endothelin-1-mediated protection against oxidant stress-induced apoptosis in cardiac myocytes. Circ Res 88: 12391246,2001

252. Kalra D, Baumgarten G, Dibbs Z, Seta Y, Sivasubramanian N, and MANN DL. Nitric oxide provokes tumor necrosis factor-alpha expression in adult feline myocardium through a cGMP-dependent pathway. Circulation 102: 1302-1307, 2000.

253. KaluZa GL, Andries LJ, Sys SU, and BrutSaErt DL. Modified model of pacing-induced heart failure in rabbits: hemodynamics and morphology (Abstract). J Heart Failure 2: 358, 1995.

254. Kanai AJ, Mesaros S, Finkel MS, Oddis CV, Birder LA, And Malinsk T. Beta-adrenergic regulation of constitutive nitric oxide synthase in cardiac myocytes. Am J Physiol Cell Physiol 273: C1371-C1377, 1997.

255. Karazyn M, Horrobin DF, Manku MS, Cunnane SC, Karmali RA, Ally AI, Morgan RO, Nicolaou KC, and Barnette WE. Effect of prostacyclin on perfusion pressure, electrical activity, rate and force of contraction in isolated rat and rabbit hearts. Life Sci 22 : 2079-2085, 1978.

256. KARDAMI E AND FANDRICH RR. Basic fibroblast growth factor in atria and ventricles of the vertebrate heart. J Cell Biol 109: 1865-1875, 1989.

257. Kasai K, Hattori Y, Banba N, Hattori S, Motohashi S, Shimoda S, NAKANISHI N, AND GROSS SS. Induction of tetrahydrobiopterin synthesis in rat cardiac myocytes: impact on cytokine-induced NO generation. Am J Physiol Heart Circ Physiol 273: H665-H672, 1997.

258. Kastner P, Grondona JM, Mark M, Gansmuller A, LeMeur M,
Decimo D, Vonesch JL, Dolle P, and Chambon P. Genetic analysis of RXR alpha developmental function: convergence of RXR and RAR signaling pathways in heart and eye morphogenesis. Cell 78: 9871003, 1994.

259. Kastner P, Messaddeq N, Mark M, Wendling O, Grondona JM, Ward S, GHYSelinck N, AND Chambon P. Vitamin A deficiency and mutations of RXRalpha, RXRbeta and RARalpha lead to early differentiation of embryonic ventricular cardiomyocytes. Development 124: 4749-4758, 1997.

260. Katircioglu SF, Saritas Z, Ulus AT, Yamak B, Yucel D, and Ayaz S. Iloprost added to the cardioplegic solutions improves myocardial performance. Prostaglandins Other Lipid Mediat 55: 51-65, 1998.

261. Katz SD, Biasucci L, Sabba C, Strom Ja, Jondeau G, Galvao M, Solomon S, Nikolic SD, Forman R, and LeJemtel TH. Impaired endothelium-mediated vasodilation in the peripheral vasculature of patients with congestive heart failure. J Am Coll Cardiol 19: 918925, 1992.

262. Kaye DM, Kelly RA, AND Smith TW. Cytokines and cardiac hypertrophy: roles of angiotensin II and basic fibroblast growth factor. Clin Exp Pharmacol Physiol Suppl 3: S136-S141, 1996.

263. Kelly RA, Balligand JL, and Smith TW. Nitric oxide and cardiac function. Circ Res 79: 363-380, 1996.

264. KeYes WM AND SANDERS EJ. Cell death in the endocardial cushions of the developing heart. J Mol Cell Cardiol 31: 1015-1023, 1999.

265. Khadour FH, Kao RH, Park S, Armstrong PW, Holycross BJ, and ScHulz R. Age-dependent augmentation of cardiac endothelial NOS in a genetic rat model of heart failure. Am J Physiol Heart Circ Physiol 273: H1223-H1230, 1997.

266. Khadour FH, O’Brien DW, Fu Y, Armstrong PW, and Schulz R. Endothelial nitric oxide synthase increases in left atria of dogs with pacing-induced heart failure. Am J Physiol Heart Circ Physiol 275: H1971-H1978, 1998

267. Kichuk MR, Seyedi N, Zhang X, Marboe CC, Michler RE, Addonizio LJ, Kaley G, NASJleTti A, AND HintZE TH. Regulation of nitric oxide production in human coronary microvessels and the contribution of local kinin formation. Circulation 94: 44-51, 1996.

268. KIm SZ, CHo KW, AND KIM SH. Modulation of endocardial natriuretic peptide receptors in right ventricular hypertrophy. Am J Physiol Heart Circ Physiol 277: H2280-H2289, 1999.

269. Kirstein M, Rivet BM, Hatem S, Benardeau A, Mercadier JJ, and FischMEISTER R. Nitric oxide regulates the calcium current in isolated human atrial myocytes. J Clin Invest 95: 794-802, 1995.

270. Kitakaze M, Node K, Komamura K, Minamino T, Inoue M, Hori M, AND KAMADA T. Evidence for nitric oxide generation in the cardiomyocytes: its augmentation by hypoxia. $J$ Mol Cell Cardiol 27: 2149-2154, 1995.

271. Kitsukawa T, Shimono A, Kawakami A, Kondoh H, and Fujisawa H. Overexpression of a membrane protein, neuropilin, in chimeric mice causes anomalies in the cardiovascular system, nervous system and limbs. Development 121: 4309-4318, 1995.

272. Kuekshus H, Smiseth OA, Kuinge R, Oie E, Hystad ME, and AttraMADAL H. Regulation of ET: pulmonary release of ET contributes to increased plasma ET levels and vasoconstriction in CHF. Am J Physiol Heart Circ Physiol 278: H1299-H1310, 2000.

273. KLABunde RE, Ritger RC, ANd Helgren MC. Cardiovascular actions of inhibitors of endothelium-derived relaxing factor (nitric oxide) formation/release in anesthetized dogs. Eur J Pharmacol 199: 5159, 1991.

274. Kuimaschewski L, Kummer W, Mayer B, Couraud JY, Preissler U, Philippin B, ANd Heym C. Nitric oxide synthase in cardiac nerve fibers and neurons of rat and guinea pig heart. Circ Res 71: 15331537, 1992.

275. KNecht M, Burkhoff D, Yi GH, Popilskis S, Homma S, Packer M, AND WANG J. Coronary endothelial dysfunction precedes heart failure and reduction of coronary reserve in awake dogs. $\mathrm{J} \mathrm{Mol} \mathrm{Cell}$ Cardiol 29: 217-227, 1997.

276. Knowles RG and Moncada S. Nitric oxide as a signal in blood vessels. Trends Biochem Sci 17: 399-402, 1992.

277. Kobayashi T, Miyauchi T, Sakai S, Kobayashi M, Yamaguchi I, Goto $\mathrm{K}$, AND Sugishita Y. Expression of endothelin-1, $\mathrm{ET}_{\mathrm{A}}$ and $\mathrm{ET}_{\mathrm{B}}$ receptors, and ECE and distribution of endothelin-1 in failing rat heart. Am J Physiol Heart Circ Physiol 276: H1197-H1206, 1999.

278. Kochilas LK, Li J, Jin F, Buck CA, AND EPstein JA. p57Kip2 expres- 
sion is enhanced during mid-cardiac murine development and is restricted to trabecular myocardium. Pediatr Res 45: 635-642, 1999.

279. Koch-Weser J. Nature of the inotropic action of angiotensin on ventricular myocardium. Circ Res 16: 230-237, 1965.

280. KoHler R, Distler A, AND HoYer J. Pressure-activated cation channel in intact rat endocardial endothelium. Cardiovasc Res 38: 433440, 1998.

281. KoJDA G AND KotTENBERG K. Regulation of basal myocardial function by NO. Cardiovasc Res 41: 514-523, 1999.

282. Kojda G, Kottenberg K, Nix P, Schluter KD, Piper HM, and Noack E. Low increase in cGMP induced by organic nitrates and nitrovasodilators improves contractile response of rat ventricular myocytes. Circ Res 78: 91-101, 1996.

283. KoJda G, KotTenberg K, AND NOACK E. Inhibition of nitric oxide synthase and soluble guanylate cyclase induces cardiodepressive effects in normal rat hearts. Eur J Pharmacol 334: 181-190, 1997.

284. Kojima M, Shiojima I, Yamazaki T, Komuro I, Zou Z, Wang Y, Mizuno T, UeKI K, TOBE K, AND KadowaKI T. Angiotensin II receptor antagonist TCV-116 induces regression of hypertensive left ventricular hypertrophy in vivo and inhibits the intracellular signaling pathway of stretch-mediated cardiomyocyte hypertrophy in vitro. Circulation 89: 2204-2211, 1994.

285. Kostetski I, Jiang Y, Kostetskaia E, Yuan S, Evans T, and Zile M. Retinoid signaling required for normal heart development regulates GATA-4 in a pathway distinct from cardiomyocyte differentiation. Dev Biol 206: 206-218, 1999.

286. Kramer BK, Smith TW, and Kelly RA. Endothelin and increased contractility in adult rat ventricular myocytes. Role of intracellular alkalosis induced by activation of the protein kinase C-dependent $\mathrm{Na}^{+}-\mathrm{H}^{+}$exchanger. Circ Res 68: 269-279, 1991.

287. Kramer R, Bucay N, Kane DJ, Martin LE, Tarpley JE, and Theili LE. Neuregulins with an Ig-like domain are essential for mouse myocardial and neuronal development. Proc Natl Acad Sci USA 93: 4833-4838, 1996.

288. KROLL J AND WALTENBERGER J. VEGF-A induces expression of eNOS and iNOS in endothelial cells via VEGF receptor-2 (KDR). Biochem Biophys Res Commun 252: 743-746, 1998.

289. Ku DD, NELSON JM, Caulfield JB, AND Winn MJ. Release of endothelium-derived relaxing factors from canine cardiac valves. $J$ Cardiovasc Pharmacol 16: 212-218, 1990.

290. Ku DD, Zaleski JK, Liu S, AND BRock TA. Vascular endothelial growth factor induces EDRF-dependent relaxation in coronary arteries. Am J Physiol Heart Circ Physiol 265: H586-H592, 1993.

291. Kubo SH, Rector TS, Bank AJ, Williams RE, and Heifetz SM. Endothelium-dependent vasodilation is attenuated in patients with heart failure. Circulation 84: 1589-1596, 1991.

292. Kubota I, Han X, Opel DJ, Zhao YY, Baliga R, Huang P, Fishman MC, Shannon RP, Michel T, AND KeLLY RA. Increased susceptibility to development of triggered activity in myocytes from mice with targeted disruption of endothelial nitric oxide synthase. J Mol Cell Cardiol 32: 1239-1248, 2000.

293. Kubota T, McTiernan CF, Frye CS, Slawson SE, Lemster BH, KoRETSKY AP, Demetris AJ, AND FeLdMAN AM. Dilated cardiomyopathy in transgenic mice with cardiac-specific overexpression of tumor necrosis factor-alpha. Circ Res 81: 627-635, 1997.

294. Kuhl U, Noutsias M, SeEberg B, and Schultheiss HP. Immunohistological evidence for a chronic intramyocardial inflammatory process in dilated cardiomyopathy. Heart 75: 295-300, 1996.

295. KuMAR S, WEST DC, AND AGER A. Heterogeneity in endothelial cells from large vessels and microvessels. Differentiation 36: 57-70, 1987.

296. Kurihara Y, Kurihara H, Oda H, MaEmura K, Nagai R, Ishikawa T, AND YAZAKI Y. Aortic arch malformations and ventricular septal defect in mice deficient in endothelin-1. J Clin Invest 96: 293-300, 1995.

297. Kurihara Y, Kurihara H, Suzuki H, Kodama T, Maemura K, Nagai R, Oda H, KuWAKI T, CAO WH, AND Kamada N. Elevated blood pressure and craniofacial abnormalities in mice deficient in endothelin-1. Nature 368: 703-710, 1994.

298. KuSAKA Y, KeLLY RA, WILLIAMS GH, AND KIFOR I. Coronary microvascular endothelial cells cosecrete angiotensin II and endothelin-1 via a regulated pathway. Am J Physiol Heart Circ Physiol 279: H1087H1096, 2000
299. Kwee L, Baldwin HS, Shen HM, Stewart CL, Buck C, Buck CA, and LABOW MA. Defective development of the embryonic and extraembryonic circulatory systems in vascular cell adhesion molecule (VCAM-1) deficient mice. Development 121: 489-503, 1995.

300. Ladoux A AND Frelin C. Hypoxia is a strong inducer of vascular endothelial growth factor mRNA expression in the heart. Biochem Biophys Res Commun 195: 1005-1010, 1993.

301. LAKKIS MM AND EPSTEIN JA. Neurofibromin modulation of ras activity is required for normal endocardial-mesenchymal transformation in the developing heart. Development 125: 4359-4367, 1998.

302. Lamers WH, Viragh S, Wessels A, Moorman AF, and Anderson RH. Formation of the tricuspid valve in the human heart. Circulation 91: 111-121, 1995.

303. LANCASTER J. Nitric oxide. Sources and detection of NO: NO synthase. In: Methods in Enzymology, edited by Packer L. New York: Academic, 1996, p. 31-50.

304. Lang D, Mosfer SI, Shakesby A, Donaldson F, and Lewis MJ. Coronary microvascular endothelial cell redox state in left ventricular hypertrophy: the role of angiotensin II. Circ Res 86: 463-469, 2000.

305. Lankes WT, Czelik C, DieTz R, and Birchmeier C. Neuregulin and ErbB receptors in cardiac development (Abstract). Circulation 100 Suppl I: I-41, 1999.

306. Lariviere R, Deng LY, Day R, Sventek P, Thibault G, and Schiffrin EL. Increased endothelin-1 gene expression in the endothelium of coronary arteries and endocardium in the DOCA-salt hypertensive rat. J Mol Cell Cardiol 27: 2123-2131, 1995.

307. Laskey RE, Adams DJ, Johns A, Rubanyi GM, and van Breemen C. Membrane potential and $\mathrm{Na}^{+}-\mathrm{K}^{+}$pump activity modulate resting and bradykinin-stimulated changes in cytosolic free calcium in cultured endothelial cells from bovine atria. J Biol Chem 265: 2613-2619, 1990.

308. Lee KF, Simon H, Chen H, Bates B, Hung MC, and Hauser C. Requirement for neuregulin receptor erbB2 in neural and cardiac development. Nature 378: 394-398, 1995.

309. Lee RRK, Stainier DYR, Weinstein BM, and Fishman MC. Cardiovascular development in the zebrafish. II. Endocardial progenitors are sequestered within the heart field. Development 120: 3361-3366, 1994.

310. Lee TC, Zhao YD, Courtman DW, and Stewart DJ. Abnormal aortic valve development in mice lacking endothelial nitric oxide synthase. Circulation 101: 2345-2348, 2000.

311. Lee Y, Song AJ, Baker R, Micales B, Conway SJ, and Lyons GE. Jumonji, a nuclear protein that is necessary for normal heart development. Circ Res 86: 932-938, 2000

312. LeJemtel TH, Maskin CS, Lucido D, and Chadwick BJ. Failure to augment maximal limb blood flow in response to one-leg versus two-leg exercise in patients with severe heart failure. Circulation 74: 245-251, 1986.

313. LELKES PI. Conference report: endothelial cell heterogeneity and organ specificity. Endothelium 1: 69-70, 1993.

314. Leskinen H, Vuolteenaho O, LePpaluoto J, and Ruskoaho H. Role of nitric oxide on cardiac hormone secretion: effect of $N^{\mathrm{G}}$-nitro-Larginine methyl ester on atrial natriuretic peptide and brain natriuretic peptide release. Endocrinology 136: 1241-1249, 1995

315. Letterio JJ, Geiser AG, Kulkarni AB, Roche NS, Sporn MB, AND ROBERTS AB. Maternal rescue of transforming growth factor-beta 1 null mice. Science 264: 1936-1938, 1994.

316. LeVIN ER. Endothelins. N Engl J Med 333: 356-363, 1995.

317. Levy AP, Levy NS, Loscalzo J, Calderone A, Takahashi N, Yeo KT, Koren G, COLUCCI WS, AND GoldBerg MA. Regulation of vascular endothelial growth factor in cardiac myocytes. Circ Res 76: 758766, 1995.

318. LEW RA AND BAERTSCHI AJ. Endothelial cells stimulate ANF secretion from atrial myocytes in co-culture. Biochem Biophys Res Commun 163: 701-709, 1989.

319. LEW RA AND BAERTSCHI AJ. Mechanisms of hypoxia-induced atrial natriuretic factor release from rat hearts. Am J Physiol Heart Circ Physiol 257: H147-H156, 1989.

320. Ley K, Gaehtgens P, and Spanel BK. Differential adhesion of granulocytes to five distinct phenotypes of cultured microvascular endothelial cells. Microvasc Res 43: 119-133, 1992.

321. Li J, Brown LF, HibBerd MG, Grossman JD, Morgan JP, and Simons M. VEGF, flk-1, and flt-1 expression in a rat myocardial infarction 
model of angiogenesis. Am J Physiol Heart Circ Physiol 270: H1803-H1811, 1996.

322. Li J, Hampton T, Morgan JP, AND Simons M. Stretch-induced VEGF expression in the heart. $J$ Clin Invest 100: 18-24, 1997.

323. Li K, Rouleau JL, ANDries LJ, and BRutSaERT DL. Effect of dysfunctional vascular endothelium on myocardial performance in isolated papillary muscles. Circ Res 72: 768-777, 1993.

324. Li K, Rouleau JL, Calderone A, Andries JL, and Brutsaert DL. Endocardial function in pacing-induced heart failure in the dog. $J$ Mol Cell Cardiol 25: 529-540, 1993.

325. Li K, Sirois P, and Rouleau JL. Role of endothelial cells in cardiovascular function. Life Sci 54: 579-592, 1994.

326. LI K, SteWART DJ, AND RoulEAu JL. Myocardial contractile actions of endothelin-1 in rat and rabbit papillary muscles. Role of endocardial endothelium. Circ Res 69: 301-312, 1991.

327. Liao W, Bisgrove BW, Sawyer H, Hug B, Bell B, Peters K, GrunWALD DJ, AND STAINIER DY. The zebrafish gene cloche acts upstream of a flk-1 homologue to regulate endothelial cell differentiation. Development 124: 381-389, 1997.

328. Lin Q, Schwarz J, Bucana C, and Olson EN. Control of mouse cardiac morphogenesis and myogenesis by transcription factor MEF2C. Science 276: 1404-1407, 1997.

329. LINASK KK AND LASH JW. Early heart development: dynamics of endocardial cell sorting suggests a common origin with cardiomyocytes. Dev Dyn 196: 62-69, 1993.

330. Linden RJ AND Kappagoda CT. Atrial receptors. In: Monographs of the Physiological Society. Cambridge, UK: Cambridge Univ. Press, 1982, vol. 39, p. 6-30.

331. Linz W, Martorana PA, Grotsch H, Bei-Yin Q, and Scholkens BA. Antagonising bradykinin (BK) obliterates the cardioprotective effects of bradykinin and angiotensin-converting enzyme (ACE) inhibitors in ischemic hearts. Drug Dev Res 19: 393-408, 1990.

332. Linz W AND Scholkens BA. A specific B2-bradykinin receptor antagonist HOE 140 abolishes the antihypertrophic effect of ramipril. Br J Pharmacol 105: 771-772, 1992.

333. Linz W, Wiemer G, AND Scholkens BA. ACE-inhibition induces NOformation in cultured bovine endothelial cells and protects isolated ischemic rat hearts. J Mol Cell Cardiol 24: 909-919, 1992.

335. LiU H AND LEE SS. Cardiopulmonary dysfunction in cirrhosis. $J$ Gastroenterol Hepatol 14: 600-608, 1999.

336. Liu PP, Dawood F, Siu S, Rouleau JL, Wen WH, and Jamerski M. The role of coronary microvascular integrity in LV remodeling and function post myocardial infarction (Abstract). Circulation 100: I-55, 1999.

337. Loke KE, Laycock SK, Mital S, Wolin MS, Bernstein R, Oz M, AdDONIZIO L, KALEY G, and Hintze TH. Nitric oxide modulates mitochondrial respiration in failing human heart. Circulation 100: 1291-1297, 1999.

338. Lombes M, Oblin ME, Gasc JM, Baulieu EE, Farman N, and BonvaLET JP. Immunohistochemical and biochemical evidence for a cardiovascular mineralocorticoid receptor. Circ Res 71: 503-510, 1992.

339. Lough J and Sugi Y. Endoderm and heart development. Dev Dyn 217: 327-342, 2000 .

340. Lum H AND MALIK AB. Mechanisms of increased endothelial permeability. Can J Physiol Pharmacol 74: 787-800, 1996.

341. Luscher TF, Boulanger CM, Yang Z, Noll G, AND Dohi Y. Interactions between endothelium-derived relaxing and contracting factors in health and cardiovascular disease. Circulation 87 Suppl V: V-36-V-44, 1993.

342. Luscher TF AND Noll G. Endothelium dysfunction in the coronary circulation. J Cardiovasc Pharmacol 24 Suppl 3: S16-S26, 1994.

343. Lymboussaki A, Olofsson B, ERiksson U, and Alitalo K. Vascular endothelial growth factor (VEGF) and VEGF-C show overlapping binding sites in embryonic endothelia and distinct sites in differentiated adult endothelia. Circ Res 85: 992-999, 1999.

343a.MacCarthy PA, Grieve DJ, Li J-M, Dunster C, Kelly FJ, and ShaH AM. Impaired endothelial regulation of ventricular relaxation in cardiac hypertrophy. Role of reactive oxygen species and NADPH oxidase. Circulation 104: 2967-2974, 2001.

344. MACCARTHY PA AND SHAH AM. Impaired endothelium-dependent regulation of ventricular relaxation in pressure-overload cardiac hypertrophy. Circulation 101: 1854-1860, 2000.

344a.Mackenzie L, Bootman M-D, Laine M, Berridge M-J, Thuring J,
Holmes A, Li W-H, AND LIPP P. The role of inositol 1,4,5-trisphosphate receptors in $\mathrm{Ca}^{2+}$ signalling and the generation of arrhythmias in rat atrial myocytes. J Physiol 541: 395-409, 2002.

345. Mahmarian JJ, Moye LA, Chinoy DA, Sequeira RF, Habib GB, Henry WJ, Jain A, Chaitman BR, Weng CS, Morales BH, and PRatt CM. Transdermal nitroglycerin patch therapy improves left ventricular function and prevents remodeling after acute myocardial infarction: results of a multicenter prospective randomized, double-blind, placebo-controlled trial. Circulation 97: 2017-2024, 1998.

346. Mahmood R, Flanders KC, AND MorRiss-Kay GM. Interactions between retinoids and TGF beta in mouse morphogenesis. Development 115: 67-74, 1992.

347. Maisonpierre PC, Suri C, Jones PF, Bartunkova S, Wiegand SJ, RAdZiejewski C, COMpton D, McClain J, ALdRich TH, PaPAdopoulos N, Daly TJ, Davis S, Sato TN, and Yancopollos GD. Angiopoietin-2, a natural antagonist for Tie2 that disrupts in vivo angiogenesis. Science 277: 55-60, 1997.

348. MAJKA SM AND McGuiRe PG. Regulation of urokinase expression in the developing avian heart: a role for the Ets-2 transcription factor. Mech Dev 68: 127-137, 1997.

349. MAJumder K AND OverbeEK PA. Left-right asymmetry and cardiac looping. In: Heart Development, edited by Harvey R and Rosenthal N. New York: Academic, 1999, p. 391-402.

350. Manabe K, Ito H, Matsuda H, Noma A, and Shibata Y. Classification of ion channels in the luminal and abluminal membranes of guineapig endocardial endothelial cells. J Physiol 484: 41-52, 1995.

351. Manabe K, TaKano M, and Noma A. Non-selective cation current of guinea-pig endocardial endothelial cells. J Physiol 487: 407-419, 1995.

352. ManaseK FJ. Embryonic development of the heart. I. A light and electron microscopic study of myocardial development in the early chick embryo. J Morphol 125: 329-365, 1968.

353. MandRiota SJ AND PepPer MS. Regulation of angiopoietin-2 mRNA levels in bovine microvascular endothelial cells by cytokines and hypoxia. Circ Res 83: 852-859, 1998.

354. Manduteanu I, Popov D, Radu A, and Simionescu M. Calf cardiac valvular endothelial cells in culture: production of glycosaminoglycans, prostacyclin and fibronectin. J Mol Cell Cardiol 20: 103-118, 1988.

355. MANOlopoulos VG AND Lelkes PI. Cyclic strain and forskolin differentially induce cAMP production in phenotypically diverse endothelial cells. Biochem Biophys Res Commun 191: 1379-1385, 1993.

356. MARChionni MA. Cell-cell signaling. Neu tack on neuregulin. Nature 378: 334-335, 1995.

357. Marfella R and Giugliano D. Stress hyperglycaemia and death after myocardial infarction. Lancet 355: 1647, 2000.

358. MariJianowski MM, van LaAr M, Bras J, and Becker AE. Chronic congestive heart failure is associated with a phenotypic shift of intramyocardial endothelial cells. Circulation 92: 1494-1498, 1995.

359. Marron K, Wharton J, Sheppard MN, Fagan D, Royston D, Kuhn DM, DE LEval MR, Whitehead BF, ANDERson RH, and Polak JM. Distribution, morphology, and neurochemistry of endocardial and epicardial nerve terminal arborizations in the human heart. Circulation 92: 2343-2351, 1995.

360. Maruyama K, Mori Y, Murasawa S, Masaki H, Takahashi N, Tsutusmi Y, Moriguchi Y, Shibazaki Y, Tanaka Y, Shibuya M, Inada M, MatSUBARA H, AND IWASAKA T. Interleukin-1 beta upregulates cardiac expression of vascular endothelial growth factor and its receptor $\mathrm{KDR} / \mathrm{flk}-1$ via activation of protein tyrosine kinases. $\mathrm{J}$ Mol Cell Cardiol 31: 607-617, 1999.

361. Masuda H, Kawamura K, Tohda K, Shozawa T, Sageshima M, and Honma M. Endocardium of the left ventricle in volume-loaded canine heart. A histological and ultrastructural study. Acta Pathol Jpn 39: 111-120, 1989.

362. Mathier MA, Rose GA, Fifer MA, Miyamoto Mi, Dinsmore RE, Castano HH, Dec GW, Palacios IF, and Semigran MJ. Coronary endothelial dysfunction in patients with acute-onset idiopathic dilated cardiomyopathy. J Am Coll Cardiol 32: 216-224, 1998.

363. Matsuoka H, Nakata M, Kohno K, Koga Y, Nomura G, Toshima H, AND IMAIZumI T. Chronic L-arginine administration attenuates cardiac hypertrophy in spontaneously hypertensive rats. Hypertension 27: 14-18, 1996. 
364. Mayer B and Hemmens B. Biosynthesis and action of nitric oxide in mammalian cells. Trends Biochem Sci 22: 477-481, 1997.

365. Mazzolai L, Nussberger J, Aubert JF, Brunner DB, Gabbiani G, BRunNer HR, AND PEDRAZZINI T. Blood pressure-independent cardiac hypertrophy induced by locally activated renin-angiotensin system. Hypertension 31: 1324-1330, 1998.

366. McClellan G, Weisberg A, Rose D, and Winegrad S. Endothelial cell storage and release of endothelin as a cardioregulatory mechanism. Circ Res 75: 85-96, 1994.

367. McDonald KM, Francis GS, Matthews J, Hunter DW, and Cohn JN. Chronic oral nitrate therapy prevents long-term ventricular remodeling in the dog. J Am Coll Cardiol 21: 514-522, 1993.

368. McDonald KM, Mock J, D'Aloia A, Parrish T, Hauer K, Francis G, StILlman A, AND CoHN JN. Bradykinin antagonism inhibits the antigrowth effect of converting enzyme inhibition in the dog myocardium after discrete transmural myocardial necrosis. Circulation 91: 2043-2048, 1995.

369. McGuire PG ANd ALEXANder SM. Inhibition of urokinase synthesis and cell surface binding alters the motile behavior of embryonic endocardial-derived mesenchymal cells in vitro. Development 118: 931-939, 1993

370. Mebazaa A, De Keulenaer GW, Paqueron X, Andries LJ, Ratajczak P, Lanone S, Frelin C, Longrois D, Payen D, Brutsaert DL, and Sys SU. Activation of cardiac endothelium as a compensatory component in endotoxin-induced cardiomyopathy: role of endothelin, prostaglandins and nitric oxide. Circulation 104: 3137-3144, 2001.

371. Mebazaa A, Martin LD, Robotham JL, Maeda K, Gabrielson EW, and WETZEL RC. Right and left ventricular cultured endocardial endothelium produces prostacyclin and $\mathrm{PGE}_{2}$. J Mol Cell Cardiol 25: 245-248, 1993.

372. MebazaA A, Mayoux E, Maeda K, Martin LD, Lakatta EG, Robotham JL, AND SHAH AM. Paracrine effects of endocardial endothelial cells on myocyte contraction mediated via endothelin. Am J Physiol Heart Circ Physiol 265: H1841-H1846, 1993.

373. Mebazaa A, Wetzel R, Cherian M, and Abraham M. Comparison between endocardial and great vessel endothelial cells: morphology, growth, and prostaglandin release. Am J Physiol Heart Circ Physiol 268: H250-H259, 1995.

374. Mebazaa A, Wetzel RC, Dodd JM, Redmond EM, Shah AM, Maeda K, Maistre G, Lakatta EG, and Robotham JL. Potential paracrine role of the pericardium in the regulation of cardiac function. Cardiovasc Res 40: 332-342, 1998.

375. Meneton P And AlHENC GF. Experimental genetic modifications in the renin-angiotensin and kallikrein-kinin systems: significance for understanding cardiovascular regulation and study of genetic determinism of human diseases. Nephrologie 19: 385-389, 1998.

376. MetSa-Ketela T. Cyclic AMP-dependent and -independent effects of prostaglandins on the contraction-relaxation cycle of spontaneously beating isolated rat atria. Acta Physiol Scand 112: 481-485, 1981.

377. Meulemans AL, Andries LJ, and Brutsaert DL. Does endocardial endothelium mediate positive inotropic response to angiotensin I and angiotensin II? Circ Res 66: 1591-1601, 1990.

378. Meulemans AL, ANDRIEs LJ, AND BRutSaert DL. Endocardial endothelium mediates positive inotropic response to alpha 1-adrenoceptor agonist in mammalian heart. J Mol Cell Cardiol 22: 667-685, 1990.

379. Meulemans AL, Sipido KR, Sys SU, and Brutsaert DL. Atriopeptin III induces early relaxation of isolated mammalian papillary muscle. Circ Res 62: 1171-1174, 1988.

380. Meyer D AND Birchmeier C. Multiple essential functions of neuregulin in development. Nature 378: 386-390, 1995.

381. Mikawa T. Retroviral targeting of FGF and FGFR in cardiomyocytes and coronary vascular cells during heart development. Ann NY Acad Sci 752: 506-516, 1995.

382. Mikawa T. Cardiac lineages. In: Heart Development, edited by Harvey RP. San Diego, CA: Academic, 1999, p. 19-33.

383. Mikawa T AND Fischman DA. Retroviral analysis of cardiac morphogenesis: discontinuous formation of coronary vessels. Proc Natl Acad Sci USA 89: 9504-9508, 1992.

384. Mikawa T and Fischman DA. The polyclonal origin of myocyte lineages. Annu Rev Physiol 58: 509-521, 1996.

385. Mikawa T AND GourdiE RG. Pericardial mesoderm generates a population of coronary smooth muscle cells migrating into the heart along with ingrowth of the epicardial organ. Dev Biol 174: 221-232, 1996.

386. Mikawa T, Hyer J, Itoh N, and Wei Y. Retroviral vectors to study cardiovascular development. Trends Cardiovasc Med 6: 79-86, 1996.

387. Miquerol L, Gertsenstein M, Harpal K, Rossant J, and Nagy A. Multiple developmental roles of VEGF suggested by a LacZ-tagged allele. Dev Biol 212: 307-322, 1999.

388. Mjaatvedt CH, Yamamura H, Wessels A, Ramsdell A, Turner D, AND MARKWALD RR. Mechanisms of segmentation, septation, and remodeling of the tubular heart: endocardial cushion fate and cardiac looping. In: Heart Development, edited by Harvey RP and Rosenthal N. San Diego, CA: Academic, 1999, p. 159-177.

389. MoBert J AND Becker BF. Cyclooxygenase inhibition aggravates ischemia-reperfusion injury in the perfused guinea pig heart: involvement of isoprostanes. J Am Coll Cardiol 31: 1687-1694, 1998.

390. Mohan P, Brutsaert DL, Paulus WJ, and Sys SU. Myocardial contractile response to nitric oxide and cGMP. Circulation 93: 12231229,1996

391. Mohan P, BrutSaert DL, AND Sys SU. Positive inotropic effect of acetylcholine: role of endocardial endothelium, cGMP, nitric oxide, prostaglandins (Abstract). Circulation 90: I-649, 1994.

392. MoHAN P, BRUTSAERT DL, AND SYs SU. Myocardial performance is modulated by interaction of cardiac endothelium derived nitric oxide and prostaglandins. Cardiovasc Res 29: 637-640, 1995.

393. Mohan P, Brutsaert DL, AND Sys SU. Positive inotropic effect of bradykinin: role of cardiac endothelium, nitric oxide, prostaglandins and endothelin (Abstract). $J$ Am Coll Cardiol 31: 108A, 1998.

394. Mohan RM, Golding S, AND PATERson DJ. Intermittent hypoxia modulates nNOS expression and heart rate response to sympathetic nerve stimulation. Am J Physiol Heart Circ Physiol 281: H132-H138, 2001.

395. Molenaar P, O’Reilly G, Sharkey A, Kuc RE, Harding DP, Plumpton C, Gresham GA, AND DAvenPort AP. Characterization and localization of endothelin receptor subtypes in the human atrioventricular conducting system and myocardium. Circ Res 72: 526-538, 1993.

396. Moncada S, Palmer RM, and Higgs EA. Nitric oxide: physiology, pathophysiology, and pharmacology. Pharmacol Rev 43: 109-142, 1991.

397. Montalescot G, Drobinski G, Meurin P, Maclouf J, Sotirov I Philippe F, Choussat R, Morin E, and Thomas D. Effects of prostacyclin on the pulmonary vascular tone and cardiac contractility of patients with pulmonary hypertension secondary to end-stage heart failure. Am J Cardiol 82: 749-755, 1998.

398. Moorman AF, DE Jong F, Denyn MM, and Lamers WH. Development of the cardiac conduction system. Circ Res 82: 629-644, 1998.

399. Morgan J, Smith JA, Wilkins GM, and Leake DS. Oxidation of low density lipoprotein by bovine and porcine aortic endothelial cells and porcine endocardial cells in culture. Atherosclerosis 102: 209$216,1993$.

400. Morimoto T, Hasegawa K, Kaburagi S, Kakita T, Wada H, Yanazume $\mathrm{T}$, AND SASAYAMA S. Phosphorylation of GATA-4 is involved in alpha 1-adrenergic agonist-responsive transcription of the endothelin-1 gene in cardiac myocytes. J Biol Chem 275: 13721-13726, 2000.

401. Mosseri M, Schaper J, Admon D, Hasin Y, Gotsman MS, Sapoznikov D, PICKERING JG, AND YAROM R. Coronary capillaries in patients with congestive cardiomyopathy or angina pectoris with patent main coronary arteries. Ultrastructural morphometry of endomyocardial biopsy samples. Circulation 84: 203-210, 1991.

402. MURAD F. Cyclic guanosine monophosphate as a mediator of vasodilation. J Clin Invest 78: 1-5, 1986.

403. Murakoshi N, Miyauchi T, Kisanuki Y, Kakinuma Y, Yuki K, YamaguCHI I, Goto K, AND YanaGisawa M. Cardiac hypertrophic response by acute pressure-overload is reduced in cardiomyocyte-specific endothelin-1 knockout mice in which coronary endothelial endothelin-1 is not knocked out (Abstract). Circulation 104: II-86, 2001.

404. Murphy J-F and FitZGERALD D-J. Vascular endothelial growth factor induces cyclooxygenase-dependent proliferation of endothelial cells via the VEGF-2 receptor. FASEB $J$ 15: 1667-1669, 2001.

405. Nagasawa T, Hirota S, Tachibana K, TakakURa N, Nishikawa S, Kitamura Y, Yoshida N, Kikutani H, and Kishimoto T. Defects of 
B-cell lymphopoiesis and bone-marrow myelopoiesis in mice lacking the CXC chemokine PBSF/SDF-1. Nature 382: 635-638, 1996.

406. NAKAJIMA Y, KRUg EL, AND MARKWALD RR. Myocardial regulation of transforming growth factor-beta expression by outflow tract endothelium in the early embryonic chick heart. Dev Biol 165: 615-626, 1994.

407. Nakajima Y, Miyazono K, Kato M, Takase M, Yamagishi T, and NAKAMURA H. Extracellular fibrillar structure of latent TGF beta binding protein-1: role in TGF beta-dependent endothelial-mesenchymal transformation during endocardial cushion tissue formation in mouse embryonic heart. J Cell Biol 136: 193-204, 1997.

408. NAKAJIMA Y AND NAKAMURA H. Expression of endothelin-A receptor during endocardial cushion formation in the early chick cardiogenesis. Biomed Res 20: 153-160, 1999.

409. Nakajima Y, Yamagishi T, HoKari S, and Nakamura H. Mechanisms involved in valvuloseptal endocardial cushion formation in early cardiogenesis: roles of transforming growth factor (TGF)-beta and bone morphogenetic protein (BMP). Anat Rec 258: 119-127, 2000.

410. NAKAMURA M. Peripheral vascular remodeling in chronic heart failure: clinical relevance and new conceptualization of its mechanisms. J Cardiac Failure 5: 127-138, 1999.

411. Nebigil CG, Choi DS, Dierich A, Hickel P, Le Meur M, Messaddeq N, LAUNAY JM, aNd MAROTEAUX L. Serotonin 2B receptor is required for heart development. Proc Natl Acad Sci USA 97: 9508-9513, 2000.

412. Nebigil CG, Hickel P, Messaddeq N, Vonesch JL, Douchet MP, Monassier L, György K, Matz R, Andriantsitohaina R, Manivet P, LAUNAY JM, AND MAROTEAUX L. Ablation of serotonin 5 - $\mathrm{HT}_{2 \mathrm{~B}}$ receptors in mice leads to abnormal cardiac structure and function Circulation 103: 2973-2979, 2001.

413. Neglia D, Parodi O, Gallopin M, Sambuceti G, Giorgetti A, Pratali L, Salvadori P, Michelassi C, Lunardi M, and Pelosi G. Myocardial blood flow response to pacing tachycardia and to dipyridamole infusion in patients with dilated cardiomyopathy without overt heart failure. A quantitative assessment by positron emission tomography. Circulation 92: 796-804, 1995.

414. Nishida M, SpRinghorn JP, Kelly RA, and Smith TW. Cell-cell signaling between adult rat ventricular myocytes and cardiac microvascular endothelial cells in heterotypic primary culture. J Clin Invest 91: 1934-1941, 1993.

415. Nishimura T, Yamada H, Kinoshita M, AND OCHI J. Endothelin expression during rat heart development: an immunohistochemical and in situ hybridization study. Biomed Res 15: 291-298, 1994.

416. Nolan GP. Cardiac development. Transcription and the broken heart. Nature 392: 129-130, 1998.

417. Nosaka S, Hashimoto M, Sasaki T, Ku K, Saitoh $\mathrm{Y}$, Hanada $\mathrm{T}$, Yamauchi M, Masumura S, NaKaYama K, and TAMURA K. Antithrombotic effects of endocardial endothelial cells: comparison with coronary artery endothelial cells. Prostaglandins 53: 305-319, 1997.

418. Nosaka S, Hashimoto M, Sasaki T, Ku K, Saitoh Y, Yamauchi M, Tanabe Y, Nakaki T, Hishikawa K, Masumura S, Nakayama K, and TAMURA K. The effects of transmural pressure on prostacyclin release from porcine endocardial endothelial cells: comparison with vascular endothelial cells. Pflügers Arch 433: 848-850, 1997.

419. Nosaka S, Nakayama K, Hashimoto M, Sasaki T, Gu K, SaitoH Y, Kin S, Yamauchi M, Masumura S, and Tamura K. Inhibition of platelet aggregation by endocardial endothelial cells. Life Sci 59: 559-564, 1996.

420. Numaguchi K, Egashira K, Takemoto M, Kadokami T, Shimokawa H, SuEISHI K, AND TAKEShITA A. Chronic inhibition of nitric oxide synthesis causes coronary microvascular remodeling in rats. Hypertension 26: 957-962, 1995.

421. Oechslin EN, Jost CH, Rojas JR, Kaufmann PA, and Jenni R. Longterm follow-up of 34 adults with isolated left ventricular noncompaction: a distinct cardiomyopathy with poor prognosis. $J$ Am Coll Cardiol 36: 493-500, 2000.

422. Oh H, Takagi H, Suzuma K, Otani A, Matsumura M, and Honda Y. Hypoxia and vascular endothelial growth factor selectively upregulate angiopoietin-2 in bovine microvascular endothelial cells. J Biol Chem 274: 15732-15739, 1999

423. Okada H, Woodcock MJ, Mitchell J, Sakamoto T, Marutsuka K, SOBEL BE, AND FUJII S. Induction of plasminogen activator inhibitor type 1 and type 1 collagen expression in rat cardiac microvascular endothelial cells by interleukin-1 and its dependence on oxygencentered free radicals. Circulation 97: 2175-2182, 1998.

424. O'Murchu B, Miller VM, Perrella MA, and Burnett JC JR. Increased production of nitric oxide in coronary arteries during congestive heart failure. J Clin Invest 93: 165-171, 1994.

425. Ono K, Еto K, Sakamoto A, Masaki T, Shibata K, Sada T, Hashimoto $\mathrm{K}$, AND Tsuлmмото G. Negative chronotropic effect of endothelin 1 mediated through $\mathrm{ET}_{\mathrm{A}}$ receptors in guinea pig atria. Circ Res 76: 284-292, 1995

426. Ono K, Tsujimoto G, Sakamoto A, Eto K, Masaki T, Ozaki Y, and SATAKE M. Endothelin-A receptor mediates cardiac inhibition by regulating calcium and potassium currents. Nature 370: 301-304, 1994.

427. Ontkean M, Gay R, and Greenberg B. Diminished endotheliumderived relaxing factor activity in an experimental model of chronic heart failure. Circ Res 69: 1088-1096, 1991.

428. ORAL H, Dorn GW, and ManN DL. Sphingosine mediates the immediate negative inotropic effects of tumor necrosis factor-alpha in the adult mammalian cardiac myocyte. J Biol Chem 272: 48364842, 1997.

429. Ozcelik C, Erdmann B, Pilz B, Osterziel KJ, Birchmeier C, and GARRATT A. Impact of the erbB2 gene on the etiology of dilated cardiomyopathy in adult mice (Abstract). Circulation 104: II-84, 2001.

430. PABLA R AND CuRTis MJ. Effects of NO modulation on cardiac arrhythmias in the rat isolated heart. Circ Res 77: 984-992, 1995.

431. Page E, Upshaw EJ, and GoINGS G. Permeability of rat atrial endocardium, epicardium, and myocardium to large molecules. Stretchdependent effects. Circ Res 71: 159-173, 1992.

432. Palmer RM, Ferrige AG, and Moncada S. Nitric oxide release accounts for the biological activity of endothelium-derived relaxing factor. Nature 327: 524-526, 1987.

433. Papapetropoulos A, Desai KM, Rudic RD, Mayer B, Zhang R, RuizTorres MP, Garcia CG, Madri JA, and Sessa WC. Nitric oxide synthase inhibitors attenuate transforming-growth-factor-beta 1-stimulated capillary organization in vitro. Am J Pathol 150: 18351844, 1997.

434. Papapetropoulos A, Garcia CG, Madri JA, and Sessa WC. Nitric oxide production contributes to the angiogenic properties of vascular endothelial growth factor in human endothelial cells. J Clin Invest 100: 3131-3139, 1997.

435. PARRATT JR. Endogenous myocardial protective (antiarrhythmic) substances. Cardiovasc Res 27: 693-702, 1993.

436. PARRATT JR. Cardioprotection by angiotensin converting enzyme inhibitors: the experimental evidence. Cardiovasc Res 28: 183-189, 1994.

437. Parratt JR AND Vegh A. Delayed protection against ventricular arrhythmias by cardiac pacing. Heart 78: 423-425, 1997.

438. Partanen J, Puri MC, Schwartz L, Fischer KD, Bernstein A, And Rossant J. Cell autonomous functions of the receptor tyrosine kinase TIE in a late phase of angiogenic capillary growth and endothelial cell survival during murine development. Development 122: 3013-3021, 1996.

439. Partanen ta, Makinen T, Arola J, Suda T, Weich Ha, and Alitalo K. Endothelial growth factor receptors in human fetal heart. Circulation 100: 583-586, 1999.

440. Pasini B, Ceccherini I, and Romeo G. RET mutations in human disease. Trends Genet 12: 138-144, 1996.

441. Paul K, Ball NA, Dorn GW, and Walsh RA. Left ventricular stretch stimulates angiotensin II-mediated phosphatidylinositol hydrolysis and protein kinase $\mathrm{C}$ epsilon isoform translocation in adult guinea pig hearts. Circ Res 81: 643-650, 1997.

442. PAulus WJ. Endothelial control of vascular and myocardial function in heart failure. Cardiovasc Drugs Ther 8: 437-446, 1994.

443. Paulus WJ, Frantz S, and Kelly RA. Nitric oxide and cardiac contractility in human heart failure. Time for reappraisal. Circulation 104: 2260-2262, 2001.

444. Paulus WJ, Kastner S, Pujadas P, Shah AM, Drexler H, and VANDERHEYDEN M. Left ventricular contractile effects of inducible nitric oxide synthase in the human allograft. Circulation 96: 34363442, 1997.

445. Paulus WJ And Shah AM. NO and cardiac diastolic function. Cardiovasc Res 43: 595-606, 1999. 
446. Paulus WJ, VAnTrimpont PJ, And Shah AM. Acute effects of nitric oxide on left ventricular relaxation and diastolic distensibility in humans. Assessment by bicoronary sodium nitroprusside infusion. Circulation 89: 2070-2078, 1994.

447. Paulus WJ, VAntrimpont PJ, And Shah AM. Paracrine coronary endothelial control of left ventricular function in humans. Circulation 92: 2119-2126, 1995.

448. Pearlman JD, Hibberd MG, Chuang ML, Harada K, Lopez JJ, GladSTONE SR, Friedman M, SEllke FW, and Simons M. Magnetic resonance mapping demonstrates benefits of VEGF-induced myocardial angiogenesis. Nat Med 1: 1085-1089, 1995.

449. Petroff MG, Kim SH, Pepe S, Dessy C, Marban E, Balligand JL, And SollotT SJ. Endogenous nitric oxide mechanisms mediate the stretch dependence of $\mathrm{Ca}^{2+}$ release in cardiomyocytes. Nat Cell Biol 3: 867-873, 2001.

450. Pinsky DJ, Patton S, and Mesaros S. Mechanical transduction of nitric oxide synthesis in the beating heart. Circ Res 81: 372-379, 1997.

451. Pinto YM, Tian X, Gohlke P, Unger T, and Paul M. A 40-fold increase in cardiac angiotensin converting enzyme activity fails to increase local angiotensin II concentrations (Abstract). Circulation 98 Suppl I: I-392, 1998.

452. Pittis M, Zhang X, Loke KE, Mital S, Kaley G, and Hintze TH. Canine coronary microvessel NO production regulates oxygen consumption in ecNOS knockout mouse heart. J Mol Cell Cardiol 32: 1141-1146, 2000.

453. Plendl J, Hartwell L, and Auerbach R. Organ-specific change in Dolichos biflorus lectin binding by myocardial endothelial cells during in vitro cultivation. In Vitro Cell Dev Biol 29: 25-31, 1993.

454. PoBer JS AND Cotran RS. Cytokines and endothelial cell biology. Physiol Rev 70: 427-451, 1990.

455. Podzuneit T, Els DJ, AND McCARthy J. Cyclic AMP mediated arrhythmias induced in the ischaemic pig heart. Basic Res Cardiol 76: 443-448, 1981.

456. Poelmann RE, Molin D, Wisse LJ, Gittenberger-de-Groot AC, And Groot GD. Apoptosis in cardiac development. Cell Tissue Res 301: 43-52, 2000.

457. Popov D, Sima A, Stern D, and Simionescu M. The pathomorphological alterations of endocardial endothelium in experimental diabetes and diabetes associated with hyperlipidemia. Acta Diabetol 33: 41-47, 1996.

458. Preckel B, Kojda G, Schlack W, Ebel D, Kottenberg K, Noack E, AND THAMER V. Inotropic effects of glyceryl trinitrate and spontaneous NO donors in the dog heart. Circulation 96: 2675-2682, 1997.

459. Prendergast BD, Anning PB, Lewis MJ, AND Shah AM. Regulation of left ventricular relaxation in the isolated guinea-pig heart by endogenous endothelin. Cardiovasc Res 33: 131-138, 1997.

460. Price S, Anning PB, Mitchell JA, and Evans TW. Myocardial dysfunction in sepsis: mechanisms and therapeutic implications. Eur Heart J 20: 715-724, 1999.

461. Puri MC, Partanen J, Rossant J, and Bernstein A. Interaction of the TEK and TIE receptor tyrosine kinases during cardiovascular development. Development 126: 4569-4580, 1999.

462. Qi XL, NGuyen TL, Andries L, Sys SU, And Rouleau JL. Vascular endothelial dysfunction contributes to myocardial depression in ischemia-reperfusion in the rat. Can J Physiol Pharmacol 76: 3545, 1998.

463. Qi XL, Stewart DJ, Gosselin H, Azad A, Picard P, Andries L, Sys SU, Brutsaert DL, AND Rouleau JL. Improvement of endocardial and vascular endothelial function on myocardial performance by captopril treatment in postinfarct rat hearts. Circulation 100: $1338-$ 1345,1999

464. QU R, SILver MM, AND Letarte M. Distribution of endoglin in early human development reveals high levels on endocardial cushion tissue mesenchyme during valve formation. Cell Tissue Res 292: 333-343, 1998

465. Radomski MW, PALmer RM, AND MoncadA S. Endogenous nitric oxide inhibits human platelet adhesion to vascular endothelium. Lancet 2: 1057-1058, 1987.

466. RafF WK, Drechsel U, Scholtholt J, And Lochner W. Effect of nitroglycerin on the heart. Pfügers Arch 317: 336-343, 1970.

467. Rajagopalan S, Laursen JB, Borthayre A, Kurz S, Keiser J, Haleen
S, GIAID A, AND HARRISON DG. Role for endothelin-1 in angiotensin II-mediated hypertension. Hypertension 30: 29-34, 1997.

468. Rakusan K, Flanagan MF, Geva T, Southern J, and Van Praagh R. Morphometry of human coronary capillaries during normal growth and the effect of age in left ventricular pressure-overload hypertrophy. Circulation 86: 38-46, 1992.

469. Rakusan K, Moravec J, and Hatt PY. Regional capillary supply in the normal and hypertrophied rat heart. Microvasc Res 20: 319 326, 1980.

470. Ramaciotti C, Sharkey A, McClellan G, and Winegrad S. Endothelial cells regulate cardiac contractility. Proc Natl Acad Sci USA 89: 4033-4036, 1992.

471. Ramsdell AF, Moreno-Rodriguez RA, Wienecke MM, Sugi Y, Turner DK, MJaAtvedt CH, AND MaRKwald RR. Identification of an autocrine signaling pathway that amplifies induction of endocardial cushion tissue in the avian heart. Acta Anat Basel 162: 1-15, 1998.

472. Ranger AM, Grusby MJ, Hodge MR, Gravallese EM, DE la Brousse FC, Hoey T, Mickanin C, Baldwin HS, And Glimcher LH. The transcription factor NF-ATc is essential for cardiac valve formation. Nature 392: 186-190, 1998.

472A.Ray P-S, Sasaki H, Estrada-Hernandez T, Zu L, and Maulik N. Effects of hypoxia/reoxygenation on angiogenic factors and their tyrosine kinase receptors in the rat myocardium. Antioxid-RedoxSignal 3: 89-102, 2001.

473. Razani B, Engelman JA, Wang XB, Schubert W, Zhang XL, Marks CB, Macaluso F, Russell RG, Li M, Pestell RG, Di-Vizio D, Hou H JR, Kneitz B, Lagaud G, Christ GJ, Edelmann W, and Lisanti MP. Caveolin-1 null mice are viable but show evidence of hyperproliferative and vascular abnormalities. J Biol Chem 276: 38121-38138, 2001.

474. Recchia FA, Bernstein RD, Sehgal PB, Ferreri NR, And Hintze TH. Cytokines are not a requisite part of the pathophysiology leading to cardiac decompensation. Proc Soc Exp Biol Med 223: 47-52, 2000.

475. Recchia FA, McConnell PI, Bernstein RD, Vogel TR, Xu X, and HINTZE TH. Reduced nitric oxide production and altered myocardial metabolism during the decompensation of pacing-induced heart failure in the conscious dog. Circ Res 83: 969-979, 1998.

476. REDMOND EM, CHERIAN MN, AND WETzEL RC. 17 $\beta$-Estradiol inhibits flow- and acute hypoxia-induced prostacyclin release from perfused endocardial endothelial cells. Circulation 90: 2519-2524, 1994.

477. Rich S AND McLaughlin VV. The effects of chronic prostacyclin therapy on cardiac output and symptoms in primary pulmonary hypertension. J Am Coll Cardiol 34: 1184-1187, 1999.

478. Risau W. Development and differentiation of endothelium. Kidney Int Suppl 67: S3-S6, 1998.

479. Ritchie RH, Marsh JD, Lancaster WD, Diglio CA, AND Schiebinger RJ. Bradykinin blocks angiotensin II-induced hypertrophy in the presence of endothelial cells. Hypertension 31: 39-44, 1998.

480. Ritchie RH, Schiebinger RJ, LaPointe MC, and Marsh JD. Angiotensin II-induced hypertrophy of adult rat cardiomyocytes is blocked by nitric oxide. Am J Physiol Heart Circ Physiol 275: H1370-H1374, 1998.

481. Rohrbach S, Yan X, Weinberg EO, Hasan F, Bartunek J, Marchionni MA, AND LORELL BH. Neuregulin in cardiac hypertrophy in rats with aortic stenosis. Differential expression of erbB2 and erbB4 receptors. Circulation 100: 407-412, 1999

482. Rolla G, Brussino L, Colagrande P, Scappaticci E, Morello M, Bergerone S, Ottobrelli A, Cerutti E, Polizzi S, and Bucca C. Exhaled nitric oxide and impaired oxygenation in cirrhotic patients before and after liver transplantation. Ann Internal Med 129: 375378, 1998.

483. ROMANO LA AND RUNYAN RB. Slug is a mediator of epithelial-mesenchymal cell transformation in the developing chicken heart. Dev Biol 212: 243-254, 1999.

484. ROSENBERG RD AND AIRD WC. Vascular-bed-specific hemostasis and hypercoagulable states. $N$ Engl J Med 340: 1555-1564, 1999.

485. RuBin LL AND StADDON JM. The cell biology of the blood-brain barrier. Annu Rev Neurosci 22: 11-28, 1999.

486. Rubio R AND CEBALlos G. Role of the endothelial glycocalyx in dromotropic, inotropic, and arrythmogenic effects of coronary flow. Am J Physiol Heart Circ Physiol 278: H106-H116, 2000.

487. Ruskoaho H, Leskinen H, Magga J, Taskinen P, Mantymaa P, Vuol- 
TEENAHO O, AND LePPALUOTO J. Mechanisms of mechanical loadinduced atrial natriuretic peptide secretion: role of endothelin, nitric oxide, and angiotensin II. J Mol Med 75: 876-885, 1997.

488. Rutherford RAD, Wharton J, Gordon L, Moscoso G, Yacoub MH, AND POLAK JM. Endocardial localization and characterization of natriuretic peptide binding sites in human fetal and adult heart. Eur J Pharmacol 212: 1-7, 1992.

489. SAdoff JD, Scholz PM, AND Weiss HR. Endogenous basal nitric oxide production does not control myocardial oxygen consumption or function. Proc Soc Exp Biol Med 211: 332-338, 1996.

490. SAeKI A, RECCHIA FA, SENZAKI H, AND Kass DA. Minimal role of nitric oxide in basal coronary flow regulation and cardiac energetics of blood-perfused isolated canine heart. J Physiol 491: 455-463, 1996.

491. Sakai S, Miyauchi T, Sakurai T, Yamaguchi I, Kobayashi M, Goto K, AND Sugishita Y. Pulmonary hypertension caused by congestive heart failure is ameliorated by long-term application of an endothelin receptor antagonist. Increased expression of endothelin-1 messenger ribonucleic acid and endothelin-1-like immunoreactivity in the lung in congestive heart failure in rats. $J \mathrm{Am}$ Coll Cardiol 28: 1580-1588, 1996.

492. Sakai S, Yorikane R, Miyauchi T, Sakurai T, Kasuya Y, Yamaguchi I, Sugishita Y, AND Goto K. Altered production of endothelin-1 in the hypertrophied rat heart. J Cardiovasc Pharmacol 26 Suppl 3: S452S455, 1995.

493. SAlvEMini D. Regulation of cyclooxygenase enzymes by nitric oxide. Cell Mol Life Sci 53: 576-582, 1997.

494. Sam F, Sawyer DB, Xie Z, Chang DLF, Ngoy S, Brenner DA, Siwik DA, Singh K, APSTEIN CS, AND Colucci WS. Mice lacking inducible nitric oxide synthase have improved left ventricular contractile function and reduced apoptotic cell death late after myocardial infarction. Circ Res 89: 351-356, 2001.

495. SANDER TL, Johnson EN, Kaushal S, ANd Bischoff J. VEGF induces NFATc1 activation in valvular endothelial cells (Abstract). Circulation 104: II-28, 2001.

496. SANDERSON MJ. Intercellular waves of communication. News Physiol Sci 11: 262-269, 1996.

497. Sanford LP, Ormsby I, GitTenberger-De-Groot AC, Sariola H, Friedman R, Boivin GP, CARdell EL, and Doetschman T. TGFbeta2 knockout mice have multiple developmental defects that are nonoverlapping with other TGFbeta knockout phenotypes. Development 124: 2659-2670, 1997.

499. Sasayama S, Matsumori A, and Kihara Y. New insights into the pathophysiological role for cytokines in heart failure. Cardiovaso Res 42: 557-564, 1999.

500. Sato Tn, Tozawa Y, Deutsch U, Wolburg BK, Fujiwara Y, Gendron MM, GRIDley T, WOlBurg H, RISAU W, AND QIN Y. Distinct roles of the receptor tyrosine kinases Tie-1 and Tie-2 in blood vessel formation. Nature 376: 70-74, 1995.

501. Satoh M, Nakamura M, Tamura G, Makita S, Segawa I, Tashiro A SATODATE R, AND HiRAMORI K. Inducible nitric oxide synthase and tumor necrosis factor-alpha in myocardium in human dilated cardiomyopathy. J Am Coll Cardiol 29: 716-724, 1997.

502. Schatteman G, Motley S, Effman E, and Bowen-Pope D. Plateletderived growth factor receptor alpha subunit deleted Patch mouse exhibits severe cardiovascular dysmorphogenesis. Teratology 51: 351-366, 1995.

503. SchiafFino S. Protean patterns of gene expression in the heart conduction system. Circ Res 80: 749-750, 1997.

504. Schluter K, Katzer C, Frischkopf K, Wenzel S, Taimor G, and Piper HM. Expression, release, and biological activity of parathyroid hormone-related peptide from coronary endothelial cells. Circ Res 86: 946-951, 2000.

505. SCHLÜTER KD. PTH and PTHrP: similar structures but different functions. News Physiol Sci 14: 243-248, 1999.

507. Schneider JW, Birjiniuk VRTP, Crovello C, Carraway KL, Decaprio J, Schwab MH, AND LaI C. A potential mechanism for herceptin cardiomyopathy through abnormal ErbB2-mediated cytoskeletal signaling (Abstract). Circulation 102: II-131, 2000.

508. SCHNEIDER MD AND PARKER TG. Cardiac myocytes as targets for the action of peptide growth factors. Circulation 81: 1443-1456, 1990.

509. Schoemaker IE, Meulemans AL, Andries LJ, and Brutsaert DL. Role of endocardial endothelium in positive inotropic action of vasopressin. Am J Physiol Heart Circ Physiol 259: H1148-H1151, 1990.
510. Schror K AND Hohlfeld T. Inotropic actions of eicosanoids. Basic Res Cardiol 87: 2-11, 1992.

511. Schulz R, Smith JA, Lewis MJ, and Moncada S. Nitric oxide synthase in cultured endocardial cells of the pig. Br J Pharmacol 104: 21-24, 1991.

512. Schwartz SM, Osinska H, Setser EA, Akandi A, Klevitsky R, Davis MG, DoRn GW II, Nelson DP, and Robbins J. Cardiac specific overexpression of angiotenin converting enzyme in transgenic mice (Abstract). Circulation 98 Suppl I: I-345, 1998.

513. Schwarz P, Diem R, Dun NJ, and Forstermann U. Endogenous and exogenous nitric oxide inhibits norepinephrine release from rat heart sympathetic nerves. Circ Res 77: 841-848, 1995.

514. Sedmera D, Pexieder T, Vulllemin M, Thompson RP, and Anderson RH. Developmental patterning of the myocardium. Anat Rec 258: 319-337, 2000 .

515. Seki T, Hagiwara H, Naruse K, Kadowaki M, Kashiwagi M, Demura H, Hirose S, AND NARUSE M. In situ identification of messenger RNA of endothelial type nitric oxide synthase in rat cardiac myocytes. Biochem Biophys Res Commun 218: 601-605, 1996.

516. Seko Y, Seko Y, Takahashi N, Shibuya M, and YaZaki Y. Pulsatile stretch stimulates vascular endothelial growth factor (VEGF) secretion by cultured rat cardiac myocytes. Biochem Biophys Res Commun 254: 462-465, 1999

517. Seko Y, Takahashi N, Tobe K, Kadowaki T, and YaZaki Y. Pulsatile stretch activates mitogen-activated protein kinase (MAPK) family members and focal adhesion kinase [p125(FAK)] in cultured rat cardiac myocytes. Biochem Biophys Res Commun 259: 8-14, 1999.

518. Serneri GG, Cecioni I, Vanni S, Paniccia R, Bandinelli B, Vetere A, Janming X, Bertolozzi I, Boddi M, Lisi GF, SANi G, ANd Modesti PA. Selective upregulation of cardiac endothelin system in patients with ischemic but not idiopathic dilated cardiomyopathy: endothelin-1 system in the human failing heart. Circ Res 86: 377-385, 2000.

519. Sнан AM. Paracrine modulation of heart cell function by endothelial cells. Cardiovasc Res 31: 847-867, 1996.

520. Shah AM, Andries LJ, Meulemans AL, ANd Brutsaert DL. Endocardium modulates myocardial inotropic response to 5-hydroxytryptamine. Am J Physiol Heart Circ Physiol 257: H1790-H1797, 1989.

521. Shah AM, Brutsaert DL, Meulemans AL, ANDries LJ, AND CaPron M. Eosinophils from hypereosinophilic patients damage endocardium of isolated feline heart muscle preparations. Circulation 81: 10811088,1990

522. Shah AM, Grocott-Mason RM, Pepper CB, Mebazaa A, Henderson AH, Lewis MJ, AND Paulus WJ. The cardiac endothelium: cardioactive mediators. Prog Cardiovasc Dis 39: 263-284, 1996.

523. SHAH AM, Lewis MJ, AND HENDERSON AH. Effects of 8-bromo-cyclic GMP on contraction and on inotropic response of ferret cardiac muscle. J Mol Cell Cardiol 23: 55-64, 1991.

524. Shah AM and MacCarthy PA. Paracrine and autocrine effects of nitric oxide on myocardial function. Pharmacol Ther 86: 49-86, 2000.

525. Shah AM, MebazaA A, Wetzel RC, and Lakatta EG. Novel cardiac myofilament desensitizing factor released by endocardial and vascular endothelial cells. Circulation 89: 2492-2497, 1994.

526. Shah AM, Mebazaa A, Yang ZK, Cuda G, Lankford EB, Pepper CB, SOLLOTT SJ, SELLERS JR, ROBOTHAM JL, AND LAKATTA EG. Inhibition of myocardial crossbridge cycling by hypoxic endothelial cells: a potential mechanism for matching oxygen supply and demand? Circ Res 80: 688-698, 1997.

527. Shah AM, Meulemans AL, AND BRutSaert DL. Myocardial inotropic responses to aggregating platelets and modulation by the endocardium. Circulation 79: 1315-1323, 1989.

528. Shah AM, Spurgeon Ha, Sollott SJ, Talo A, and lakatta EG. 8-Bromo-cGMP reduces the myofilament response to $\mathrm{Ca}^{2+}$ in intact cardiac myocytes. Circ Res 74: 970-978, 1994.

529. Shalaby F, Rossant J, Yamaguchi TP, Gertsenstein M, Wu XF, BReitman ML, AND SCHUH AC. Failure of blood-island formation and vasculogenesis in Flk-1-deficient mice. Nature 376: 62-66, 1995.

530. Shen W, Tian R, Saupe KW, Spindler M, and Ingwall JS. Endogenous nitric oxide enhances coupling between $\mathrm{O}_{2}$ consumption and ATP synthesis in guinea pig hearts. Am J Physiol Heart Circ Physiol 281: H838-H846, 2001.

531. Shen W, Xu X, Ochoa M, Zhao G, Wolin MS, and Hintze TH. Role of 
nitric oxide in the regulation of oxygen consumption in conscious dogs. Circ Res 75: 1086-1095, 1994.

532. Sherman AJ, Davis CA, Klocke FJ III, Harris KR, SRInivasan G YAACOUB AS, QUINN DA, AHLIN KA, AND JANG JJ. Blockade of nitric oxide synthesis reduces myocardial oxygen consumption in vivo. Circulation 95: 1328-1334, 1997.

533. Shinke T, Takaoka H, Takeuchi M, Hata K, Kawai H, OKubo H, Kijima Y, Murata T, and Yokoyama M. Nitric oxide spares myocardial oxygen consumption through attenuation of contractile response to beta-adrenergic stimulation in patients with idiopathic dilated cardiomyopathy. Circulation 101: 1925-1930, 2000.

534. SHINOHARA K. The expression and the role of vascular endothelial growth factor (VEGF) in human normal and myocardial infarcted heart. Hokkaido Igaku Zasshi 69: 978-993, 1994

535. Shiraki H, Yoshikawa T, Anzai T, Negishi K, Takahashi T, Asakura Y, AKaishi M, Mitamura H, AND Ogawa S. Association between preinfarction angina and a lower risk of right ventricular infarction. N Engl J Med 338: 941-947, 1998.

536. Shweiki D, Itin A, Soffer D, AND Keshet E. Vascular endothelial growth factor induced by hypoxia may mediate hypoxia-initiated angiogenesis. Nature 359: 843-845, 1992.

537. Sievi E, Lahteenmaki TA, Alanko J, Vuorinen P, and VapaAtalo H. Nitric oxide as a regulator of prostacyclin synthesis in cultured rat heart endothelial cells. Arzneimittelforschung 47: 1093-1098, 1997.

538. Sigusch HH, Lehmann MH, Schnittler U, Reinhardt D, and Figulla HR. Tumour necrosis factor-alpha expression in idiopathic dilated cardiomyopathy: correlation to myocardial inflammatory activity. Cytokine 12: 1261-1266, 2000.

539. SinEY L AND LEWIS MJ. Endothelium-derived relaxing factor inhibits platelet adhesion to cultured porcine endocardial endothelium. Eur J Pharmacol 229: 223-226, 1992.

540. Smedsrod B, Pertoft H, Gustafson S, and Laurent TC. Scavenger functions of the liver endothelial cell. Biochem J 266: 313-327, 1990.

541. Smiley LM and Tyagi SC. Endocardial endothelium injury leading to fibrosis is one of the causes of cardiac failure in spontaneously hypertensive rats (Abstract). Circulation 100: I-224-I-225, 1999.

542. Smith CJ, Sun D, Hoegler C, Roth BS, Zhang X, Zhao G, Xu XB, Kobari Y, Pritchard K, Sessa WC, and Hintze TH. Reduced gene expression of vascular endothelial NO synthase and cyclooxygenase-1 in heart failure. Circ Res 78: 58-64, 1996.

543. SMITH JA, SHAH AM, AND LEWIS MJ. Factors released from endocardium of the ferret and pig modulate myocardial contraction. J Physiol 439: 1-14, 1991.

544. Sommer JR AND Johnson EA. Comparative ultrastructure of cardiac cell membrane specializations. A review. Am J Cardiol 25: $184-$ 194, 1970.

545. Song W, MaJkA SM, AND McGuire PG. Hepatocyte growth factor expression in the developing myocardium: evidence for a role in the regulation of the mesenchymal cell phenotype and urokinase expression. Dev Dyn 214: 92-100, 1999.

546. Sorensen KK, MelkKo J, And Smedsrod B. Scavenger-receptor-mediated endocytosis in endocardial endothelial cells of Atlantic cod Gadus morhua. J Exp Biol 201: 1707-1718, 1998.

547. SorIANo P. The PDGF alpha receptor is required for neural crest cell development and for normal patterning of the somites. Development 124: 2691-2700, 1997.

548. Speir E, Tanner V, Gonzalez AM, Farris J, Baird A, And Casscells W. Acidic and basic fibroblast growth factors in adult rat heart myocytes. Localization, regulation in culture, and effects on DNA synthesis. Circ Res 71: 251-259, 1992.

549. Stainier DY, Weinstein BM, Detrich HW, Zon LI III, AND Fishman MC. Cloche, an early acting zebrafish gene, is required by both the endothelial and hematopoietic lineages. Development 121: 3141$3150,1995$.

550. Stangl K, Dschietzig T, Laule M, Alexiou K, Wernecke KD, and BAUMANN G. Pulmonary big endothelin affects coronary tone and leads to enhanced, ET(A)-mediated coronary constriction in early endothelial dysfunction. Circulation 96: 3192-3200, 1997.

551. Stangl K, Dschietzig T, Richter C, Laule M, Stangl V, Tanis E, Baumann G, AND Felix SB. Pulmonary release and coronary and peripheral consumption of big endothelin and endothelin-1 in se- vere heart failure: acute effects of vasodilator therapy. Circulation 102: 1132-1138, 2000

552. Stein B, Eschenhagen T, Rudiger J, Scholz H, Forstermann U, and GATH I. Increased expression of constitutive nitric oxide synthase III, but not inducible nitric oxide synthase II, in human heart failure. J Am Coll Cardiol 32: 1179-1186, 1998.

553. Sterin-Borda L, Canga L, Borda ES, Gimeno MF, and Gimeno AL. Inotropic effect of prostacyclin $\left(\mathrm{PGI}_{2}\right)$ on isolated rat atria at different contraction frequencies. Naunyn-Schmiedebergs Arch Pharmacol 313: 95-100, 1980.

554. Stobierska-Dzierzek B, Awad H, AND Michler RE. The evolving management of acute right-sided heart failure in cardiac transplant recipients. J Am Coll Cardiol 38: 923-931, 2001.

555. STRAuER BE. Evidence for a positive inotropic effect of nitroglycerol on isolated human ventricular myocardium. Pharmacol Res Commun 3: 377-383, 1971.

556. Strauer BE AND Scherpe A. Ventricular function and coronary hemodynamics after intravenous nitroglycerin in coronary artery disease. Am Heart J 95: 210-219, 1978.

557. Stumpe T, DECKING UKM, AND Schrader J. Nitric oxide reduces energy supply by direct action on the respiratory chain in isolated cardiomyocytes. Am J Physiol Heart Circ Physiol 280: H2350H2356, 2001.

558. Sucov HM. Molecular insights into cardiac development. Annu Rev Physiol 60: 287-308, 1998.

559. Sucov HM, Dyson E, Gumeringer CL, Price J, Chien KR, And Evans RM. RXR alpha mutant mice establish a genetic basis for vitamin A signaling in heart morphogenesis. Genes Dev 8: 1007-1018, 1994.

560. SUGI Y AND MARKWALD RR. Formation and early morphogenesis of endocardial endothelial precursor cells and the role of endoderm. Dev Biol 175: 66-83, 1996.

561. Sugishita Y, Takahashi T, Shimizu T, Yao A, Kinugawa KI, Sugishita K, HaRAda K, MAtsui H, and Nagai R. Expression of genes encoding vascular endothelial growth factor and its Flk-1 receptor in the chick embryonic heart. J Mol Cell Cardiol 32: 1039-1051, 2000.

562. Suri C, Jones PF, Patan S, Bartunkova S, Maisonpierre PC, Davis S, SATO TN, AND Yancopoulos GD. Requisite role of angiopoietin-1, a ligand for the TIE2 receptor, during embryonic angiogenesis. Cell 87: 1171-1180, 1996.

563. Sys SU, De Keulenaer GW, and Brutsaert DL. Reappraisal of the multicellular preparation for the in vitro physiopharmacological evaluation of myocardial performance. Adv Exp Med Biol 453: 441-450, 1998.

564. Sys SU, De Keulenaer GW, Kaluza G, Andries LJ, and Brutsaert DL. Endocardial endothelial dysfunction. In: Endocardial Endothelium: Control of Cardiac Performance, edited by Sys SU and Brutsaert DL. Austin, TX: Landes, 1995.

565. Szabo T, Geller L, Merkely B, Selmeci L, Juhasz NA, and Solti F. Investigating the dual nature of endothelin-1: ischemia or direct arrhythmogenic effect? Life Sci 66: 2527-2541, 2000.

566. Szokodi I, Horkay F, Merkely B, Solti F, Geller L, Kiss P, Selmeci L, Kekesi V, Vuolteenaho O, Ruskoaho H, Juhasz Na, and Toth M. Intrapericardial infusion of endothelin-1 induces ventricular arrhythmias in dogs. Cardiovasc Res 38: 356-364, 1998.

567. Tada H, Thompson CI, Recchia FA, Loke KE, Ochoa M, Smith CJ, Shesely EG, Kaley G, And Hintze TH. Myocardial glucose uptake is regulated by nitric oxide via endothelial nitric oxide synthase in Langendorff mouse heart. Circ Res 86: 270-274, 2000.

568. TAKATA K, HiRANO H, AND KASAHARA M. Transport of glucose across the blood-tissue barriers. Int Rev Cytol 172: 1-53, 1997.

569. Takeshita S, Zheng LP, Brogi E, Kearney M, Pu LQ, Bunting S, Ferrara N, Symes JF, AND IsNer JM. Therapeutic angiogenesis. A single intra-arterial bolus of vascular endothelial growth factor augments revascularization in a rabbit ischemic hind limb model. J Clin Invest 93: 662-670, 1994.

570. Takeuchi Y, Kihara Y, Inagaki K, Yoneda T, and Sasayama S. Endothelin-1 has a unique oxygen-saving effect by increasing contractile efficiency in the isolated rat heart. Circulation 103: 1557-1563, 2001.

571. Talukder MAH, Norota I, SakuraI K, AND Endoh M. Inotropic response of rabbit ventricular myocytes to endothelin-1: difference from isolated papillary muscles. Am J Physiol Heart Circ Physiol 281: H596-H605, 2001. 
572. Tatsumi T, Matoba S, Kawahara A, Keira N, Shiraishi J, AKashi K, Kobara M, Tanaka T, Katamura M, Nakagawa C, Ohta B, Shirayama T, Takeda K, Asayama J, Fliss H, and Nakagawa M. Cytokineinduced nitric oxide production inhibits mitochondrial energy production and impairs contractile function in rat cardiac myocytes. J Am Coll Cardiol 35: 1338-1346, 2000.

573. TAWARA S. Das Reizleitungssystem des Saugetierherzens. Eine anatomisch-histologische Studie uber das Atrioventrikularbundel und die purkinjeschen Faden. Jena: Gustav Fischer Verlag, 1906.

574. Tessarollo L and Hempstead BL. Regulation of cardiac development by receptor tyrosine kinases. Trends Cardiovasc Med 8: $34-40,1998$

575. Tevosian SG, Deconinck AE, Tanaka M, Schinke M, Litovsky SH, Izumo S, FuJIwara Y, AND ORKIN SH. FOG-2, a cofactor for GATA transcription factors, is essential for heart morphogenesis and development of coronary vessels from epicardium. Cell 101: 729_ $739,2000$.

576. Thoenes M, Forstermann U, Tracey WR, Bleese NM, Nussler AK, Scholz H, AND Stein B. Expression of inducible nitric oxide synthase in failing and non-failing human heart. J Mol Cell Cardiol 28 : 165-169, 1996

577. Thum T and Borlak J. Gene expression in distinct regions of the heart. Lancet 355: 979-983, 2000.

578. Tobe TJ, de Langen CD, Tio RA, Bel KJ, Mook PH, and Wesseling H. In vivo effect of bradykinin during ischemia and reperfusion: improved electrical stability two weeks after myocardial infarction in the pig. J Cardiovasc Pharmacol 17: 600-607, 1991.

579. Tofukuji M, Metais C, Li J, Franklin A, Simons M, and Sellke FW. Myocardial VEGF expression after cardiopulmonary bypass and cardioplegia. Circulation 98: II-242-II-246, 1998.

580. TOMAnek RJ, Hu N, Phan B, AND Clark EB. Rate of coronary vascularization during embryonic chicken development is influenced by the rate of myocardial growth. Cardiovasc Res 41: 663$671,1999$.

581. Tomanek RJ, Lotun K, Clark EB, Suvarna PR, and Hu N. VEGF and bFGF stimulate myocardial vascularization in embryonic chick. Am J Physiol Heart Circ Physiol 274: H1620-H1626, 1998.

582. TOMANEK RJ AND RATAJSKA A. Regulation of coronary vessel formation in embryonic and fetal hearts. In: The Developing Heart, edited by Ostadal B. Philadelphia, PA: Lippincott-Raven, 1997, p. 91-100.

583. Tomanek RJ, Searls JC, and LachenBruch PA. Quantitative changes in the capillary bed during developing, peak, and stabilized cardiac hypertrophy in the spontaneously hypertensive rat. Circ Res 51: 295-304, 1982.

584. Torre AG, Kapadia S, Lee J, Durand JB, Bies RD, Young JB, and MANN DL. Tumor necrosis factor-alpha and tumor necrosis factor receptors in the failing human heart. Circulation 93: 704-711, 1996.

585. Torres M, Ceballos G, and Rubio R. Possible role of nitric oxide in catecholamine secretion by chromaffin cells in the presence and absence of cultured endothelial cells. J Neurochem 63: 988-996, 1994.

586. Towbin JA, Bowles KR, AND Bowles NE. Etiologies of cardiomyopathy and heart failure. Nat Med 5: 266-267, 1999.

587. Treasure CB, Vita JA, Cox DA, Fish RD, Gordon JB, Mudge GH, Colucci WS, Sutton MG, Selwyn AP, Alexander RW, Ganz P, AND CoLUCCI WS. Endothelium-dependent dilation of the coronary microvasculature is impaired in dilated cardiomyopathy. Circulation 81: 772-779, 1990

588. Trochu JN, Bouhour JB, Kaley G, and Hintze TH. Role of endothelium-derived nitric oxide in the regulation of cardiac oxygen metabolism. Implications in health and disease. Circ Res 87: 1108$1117,2000$.

589. Trotter JF, Box T, Heneghan M, and Shrestha R. Hetapulmonary syndrome in transplant centres at different altitudes. The Lancet 358: 336, 2001

590. Tsuchida S, Matsusaka T, Chen X, Okubo S, Nimmura F, Nishimura H, Fogo A, Utsunomiya H, Inagami T, and IchiKawa I. Murine double nullizygotes of the angiotensin type $1 \mathrm{~A}$ and $1 \mathrm{~B}$ receptor genes duplicate severe abnormal phenotypes of angiotensinogen nullizygotes. J Clin Invest 101: 755-760, 1998.

591. Turek Z, Grandtner M, Kubat K, Ringnalda BE, and Kreuzer F. Arterial blood gases, muscle fiber diameter and intercapillary dis- tance in cardiac hypertrophy of rats with an old myocardial infarction. Pflügers Arch 376: 209-215, 1978.

592. Ullrich R, Scherrer CM, Bloch KD, Ichinose F, Nakajima H, Picard MH, ZAPOL WM, AND QUEZADO ZMN. Congenital deficiency of nitric oxide synthase 2 protects against endotoxin-induced myocardial dysfunction in mice. Circulation 102: 1440-1446, 2000.

593. Urata H, Boehm KD, Philip A, Kinoshita A, Gabrovsek J, Bumpus FM, AND HuSAIN A. Cellular localization and regional distribution of an angiotensin II-forming chymase in the heart. J Clin Invest 91: 1269-1281, 1993.

594. URSell PC and MaYes M. Endothelial isoform of nitric oxide synthase in rat heart increases during development. Anat Rec 246: 465-472, 1996

595. Vandecasteele G, Eschenhagen T, Scholz H, Stein B, Verde I, and FisCHMEISTER R. Muscarinic and beta-adrenergic regulation of heart rate, force of contraction and calcium current is preserved in mice lacking endothelial nitric oxide synthase. Nat Med 5: 331-334, 1999.

596. Van den Hoff MJ, Moorman AF, RuiJter JM, Lamers WH, BenningTON RW, Markwald RR, AND Wessels A. Myocardialization of the cardiac outflow tract. Dev Biol 212: 477-490, 1999.

597. Van den Hoff MJ, van den Eujne SM, Viragh S, and Moorman AF. Programmed cell death in the developing heart. Cardiovasc Res 45: 603-620, 2000

598. Van de Voorde J And Leusen I. Role of the endothelium in the vasodilator response of rat thoracic aorta to histamine. Eur J Pharmacol 87: 113-120, 1983

599. Van Kats JP, Duncker DJ, Haitsma DB, Schuijt MP, Niebuur A, STUBENITSKY R, BOOMSMA F, SCHALEKAMP MADH, VERDOUW PD, AND DANSER AHJ. Angiotensin-converting enzyme inhibition and angiotensin II type 1 receptor blockade prevent cardiac remodeling in pigs after myocardial infarction: role of tissue angiotensin II. Circulation 102: 1556-1563, 2000.

600. VANE JR. The Croonian Lecture, 1993. The endothelium: maestro of the blood circulation. Philos Trans $R$ Soc Lond B Biol Sci 343: 225-246, 1994.

601. Vanhoutte PM. Endothelium and the control of vascular tissue. News Physiol Sci 2: 18-22, 1987.

602. VANHoutTe PM. The endothelium-modulator of vascular smoothmuscle tone. N Engl J Med 319: 512-513, 1988.

603. VANHoutTE PM. Endothelium-derived free radicals: for worse and for better. J Clin Invest 107: 23-25, 2001.

604. Vanhoutte PM, Rubany GM, Miller VM, and Houston DS. Modulation of vascular smooth muscle contraction by the endothelium. Annu Rev Physiol 48: 307-320, 1986.

605. Vaz DS, Magina S, Domingues CA, Moura D, and Guimaraes S. The role of the endocardium in the facilitatory effect of bradykinin on electrically-induced release of noradrenaline in rat cardiac ventricle. Br J Pharmacol 118: 364-368, 1996.

606. Vegh A, Papp JG, Szekeres L, and Parratt JR. Prevention by an inhibitor of the L-arginine-nitric oxide pathway of the antiarrhythmic effects of bradykinin in anaesthetized dogs. $\mathrm{Br} J$ Pharmacol 110: 18-19, 1993.

607. Vila-Petroff MG, Younes A, Egan J, Lakatta EG, and Sollott SJ. Activation of distinct cAMP-dependent and cGMP-dependent pathways by nitric oxide in cardiac myocytes. Circ Res 84: 1020-1031, 1999 .

608. Vondriska TM, Tang XL, Zhang J, Bolli R, and Ping P. Bmx, a member of the Tec family of non-receptor tyrosine kinases, is a novel participant in the signaling system of NO-induced cardioprotection (Abstract). Circulation 104: II-61, 2001.

609. Wagner OF, Vierhapper H, Gasic $\mathrm{S}$, NowOtNy P, and Waldhausl W. Regional effects and clearance of endothelin-1 across pulmonary and splanchnic circulation. Eur J Clin Invest 22: 277-282, 1992.

610. WANG J AND MoRgan JP. Endocardial endothelium modulates myofilament $\mathrm{Ca}^{2+}$ responsiveness in aequorin-loaded ferret myocardium. Circ Res 70: 754-760, 1992.

611. WANG J AND MoRGAN JP. Endothelin reverses the effects of acidosis on the intracellular $\mathrm{Ca}^{2+}$ transient and contractility in ferret myocardium. Circ Res 71: 631-639, 1992.

612. Wang J, SeYedi N, Xu XB, Wolin MS, and Hintze TH. Defective endothelium-mediated control of coronary circulation in conscious dogs after heart failure. Am J Physiol Heart Circ Physiol 266: H670-H680, 1994. 
613. WANG JX, PAIK G, AND Morgan JP. Endothelin 1 enhances myofilament $\mathrm{Ca}^{2+}$ responsiveness in aequorin-loaded ferret myocardium. Circ Res 69: 582-589, 1991.

614. WARD BJ AND DONNElly JL. Hypoxia induced disruption of the cardiac endothelial glycocalyx: implications for capillary permeability. Cardiovasc Res 27: 384-389, 1993.

615. Webb CM, Ghatei MA, McNeill JG, and Collins P. $17 \beta$-Estradiol decreases endothelin-l levels in the coronary circulation of postmenopausal women with coronary artery disease. Circulation 102: 1617-1622, 2000.

616. WebB S, Brown NA, AND ANDERSON RH. Formation of the atrioventricular septal structures in the normal mouse. Circ Res 82: 645656, 1998.

617. WeBER KT. Extracellular matrix remodeling in heart failure: a role for de novo angiotensin II generation. Circulation 96: 4065-4082, 1997.

618. Webster MW, FitzPatrick MA, Nicholls MG, Ikram H, and Wells JE. Effect of enalapril on ventricular arrhythmias in congestive heart failure. Am J Cardiol 56: 566-569, 1985.

619. Wei CM, JiAng SW, Lust JA, Daly RC, AND McGregor CGA. Genetic expression of endothelial nitric oxide synthase in human atrial myocardium. Mayo Clin Proc 71: 346-350, 1996.

620. Weil J, Eschenhagen T, Fleige G, Mittmann C, Orthey E, and ScHolz H. Localization of preproenkephalin mRNA in rat heart: selective gene expression in left ventricular myocardium. Am J Physiol Heart Circ Physiol 275: H378-H384, 1998.

621. WeitzBerg E, Ahlborg G, AND LundBerg JM. Differences in vascular effects and removal of endothelin-1 in human lung, brain, and skeletal muscle. Clin Physiol 13: 653-662, 1993.

621a.Wenzel S, Schorr K, Degenhardt H, Frischkopf K, KoJda G, Wiesner R-J, Rosenkranz S, Piper H-M, and Schluter K-D. TGFbeta1 downregulates PTHrP in coronary endothelial cells. $J \mathrm{Mol}$ Cell Cardiol 33: 1181-1190, 2001.

622. Wharton J, Rutherford RA, Gordon L, Moscoso G, Schiemberg I, GAER JA, TAYLOR KM, AND POLAK JM. Localization of endothelin binding sites and endothelin-like immunoreactivity in human fetal heart. J Cardiovasc Pharmacol 17 Suppl 7: S378-S384, 1991.

623. Wilcox JN, Augustine A, Goeddel DV, And Lowe DG. Differential regional expression of three natriuretic peptide receptor genes within primate tissues. Mol Cell Biol 11: 3454-3462, 1991.

624. Wilson JG, Roth CB, AND WARKANY J. An analysis of the syndrome of malformations induced by maternal vitamin A deficiency. Effects of restoration of vitamin A at various times during gestation. $A m J$ Anat 92: 189-217, 1953.

625. WINEGRAD S. Endothelial cell regulation of contractility of the heart. Annu Rev Physiol 59: 505-525, 1997.

626. WODITSCH I AND SCHROR K. Prostacyclin rather than endogenous nitric oxide is a tissue protective factor in myocardial ischemia. Am J Physiol Heart Circ Physiol 263: H1390-H1396, 1992.

627. Woodcock EA, Reyes N, Jacobsen AN, AND Du XJ. Inhibition of inositol(1,4,5)trisphosphate generation by endothelin-1 during postischemic reperfusion: a novel antiarrhythmic mechanism. Circulation 99: 823-828, 1999.

628. Wu H, Lee SH, GaO J, LiU X, AND IRUEla-ArIsPe ML. Inactivation of erythropoietin leads to defects in cardiac morphogenesis. Development 126: 3597-3605, 1999.

629. Wunsch AM, LitTle CD, AND Markwald RR. Cardiac endothelial heterogeneity defines valvular development as demonstrated by the diverse expression of JB3, an antigen of the endocardial cushion tissue. Dev Biol 165: 585-601, 1994.

630. WynCOLL DL AND Evans TW. Acute respiratory distress syndrome. Lancet 354: 497-501, 1999

631. Xiang JZ, Linz W, Becker H, Ganten D, Lang RE, Scholkens B, and UNGER T. Effects of converting enzyme inhibitors: ramipril and enalapril on peptide action and sympathetic neurotransmission in the isolated heart. Eur J Pharmacol 113: 215-223, 1985.

632. Xie YW, Shen W, Zhao G, Xu X, Wolin MS, and Hintze TH. Role of endothelium-derived nitric oxide in the modulation of canine myocardial mitochondrial respiration in vitro. Implications for the development of heart failure. Circ Res 79: 381-387, 1996.

633. Yamada H, Fabris B, Allen AM, Jackson B, Johnston CI, and MenDELSOHN AO. Localization of angiotensin converting enzyme in rat heart. Circ Res 68: 141-149, 1991.
634. Yamada M, Szendro PI, Prokscha A, Schwartz RJ, and Eichele G. Evidence for a role of Smad6 in chick cardiac development. Dev Biol 215: 48-61, 1999.

635. Yamagishi T, Nakajima Y, Miyazono K, and NaKamura H. Bone morphogenetic protein-2 acts synergistically with transforming growth factor-beta3 during endothelial-mesenchymal transformation in the developing chick heart. J Cell Physiol 180: 35-45, 1999.

636. Yamane A, Seetharam L, Yamaguchi S, Gotoh N, Takahashi T, Neufeld G, AND ShibuYa M. A new communication system between hepatocytes and sinusoidal endothelial cells in liver through vascular endothelial growth factor and Flt tyrosine kinase receptor family (Flt-1 and KDR/Flk-1). Oncogene 9: 2683-2690, 1994.

637. YAN M, Nick TG, AND SINNING AR. Retinoic acid inhibition of cardiac mesenchyme formation in vitro correlates with changes in the secretion of particulate matrix from the myocardium. Anat Rec 258: 186-197, 2000

638. Yan X, Converso KL, Manning WJ, Marchionni MA, and Lorell BH. Knockout of neuregulin (NRG1) promotes early heart failure in aortic stenosis mice (Abstract). Circulation 102: II-71, 2000.

639. Yan X, Ding B, WeinBerg EO, and Lorell BH. Neuregulin-erbB signaling in hypertrophied aortic stenosis mice: differential expression of ErbB2 and ErbB4 receptors (Abstract). Circulation 100: I-128, 1999

640. Yanagisawa H, Hammer RE, Richardson JA, Emoto N, Williams SC, Takeda S, Clouthier DE, and Yanagisawa M. Disruption of ECE-1 and ECE-2 reveals a role for endothelin-converting enzyme-2 in murine cardiac development. J Clin Invest 105: 1373-1382, 2000.

641. Yanagisawa H, Yanagisawa M, Kapur RP, Richardson JA, Williams SC, Clouthier DE, de Wit D, Emoto N, AND Hammer RE. Dual genetic pathways of endothelin-mediated intercellular signaling revealed by targeted disruption of endothelin converting enzyme-1 gene. Development 125: 825-836, 1998.

642. Yanagisawa M, Kurihara H, Kimura S, Tomobe Y, Kobayashi M, Mitsui Y, YazaKI Y, Goto K, AND Masaki T. A novel potent vasoconstrictor peptide produced by vascular endothelial cells. Nature 332: 411-415, 1988

643. Yang W, Ando J, Konenaga R, Toyo T, and Kamiya A. Exogenous nitric oxide inhibits proliferation of cultured vascular endothelial cells. Biochem Biophys Res Commun 203: 1160-1167, 1994.

644. YoкотA R. Occurrence of long non-myelinated axonal segments intercalated in myelinated, presumably sensory axons: electron microscopic observations in the dog atrial endocardium. J Neurocytol 13: 127-143, 1984.

645. Yost HJ. Establishing cardiac left-right asymmetry. In: Heart Development, edited by Harvey RP and Rosenthal N. San Diego, CA: Academic, 1999, p. 373-389.

646. Young LH, IKEDA Y, AND LEFER AM. Caveolin-1 peptide exerts cardioprotective effects in myocardial ischemia-reperfusion via nitric oxide mechanism. Am J Physiol Heart Circ Physiol 280: H2489H2495, 2001.

647. Yutzey K, GANnON M, AND BADER D. Diversification of cardiomyogenic cell lineages in vitro. Dev Biol 170: 531-541, 1995.

648. Zeitz CJ, Bronzwaer JGF, Heymes C, Vanderheyden M, And Paulus WJ. Left ventricular end diastolic wall stress and endomyocardial inducible nitric oxide synthase gene expression in human dilated cardiomyopathy (Abstract). J Am Coll Cardiol 35 Suppl A: 204A, 2000.

649. Zeitz CJ, Sys SU, AND BRUTSAert DL. The positive inotropic effects of hydrostatic pressure are buffered by the endocardial endothelium via prostaglandins. (Abstract). J Am Coll Cardiol 35 Suppl A: 176A-177A, 2000.

650. Zhang X, Xie YW, Nasjletti A, Xu X, Wolin MS, and Hintze TH. ACE inhibitors promote nitric oxide accumulation to modulate myocardial oxygen consumption. Circulation 95: 176-182, 1997.

651. ZHAO JJ AND LEMKE G. Selective disruption of neuregulin-1 function in vertebrate embryos using ribozyme-tRNA transgenes. Development 125: 1899-1907, 1998.

652. Zhao YY, Sawyer DR, Baliga RR, Opel DJ, Han X, Marchionni MA, AND KELLY RA. Neuregulins promote survival and growth of cardiac myocytes. Persistence of ErbB2 and ErbB4 expression in neonatal and adult ventricular myocytes. J Biol Chem 273: 10261-10269, 1998 . 
653. Zhao Z AND RivkeES SA. Programmed cell death in the developing heart: regulation by BMP4 and FGF2. Dev Dyn 217: 388-400, 2000.

654. Ziche M, Morbidelli L, ChOUdhuri R, Zhang HT, Donnini S, Granger HJ, AND BICKNELL R. Nitric oxide synthase lies downstream from vascular endothelial growth factor-induced but not basic fibroblast growth factor-induced angiogenesis. J Clin Invest 99: 2625-2634, 1997.

655. Ziche M, Morbideldi L, Masini E, Amerini S, Granger HJ, Maggi CA, GEPPETTI P, AND LEDDA F. Nitric oxide mediates angiogenesis in vivo and endothelial cell growth and migration in vitro promoted by substance P. J Clin Invest 94: 2036-2044, 1994.

656. Ziche M, Parenti A, Ledda F, Dell'Era P, Granger HJ, Maggi CA,
AND PResta M. Nitric oxide promotes proliferation and plasminogen activator production by coronary venular endothelium through endogenous bFGF. Circ Res 80: 845-852, 1997.

657. Zolk O, Quattek J, Sitzler G, Schrader T, Nickenig G, Schnabel P, SHIMADA K, TAKAHASHI M, AND BoHM M. Expression of endothelin-1, endothelin-converting enzyme, and endothelin receptors in chronic heart failure. Circulation 99: 2118-2123, 1999.

658. Zornoff LAM, Skali H, Pfeffer MA, St. John Sutton M, Rouleau JL, Lamas Ga, Plappert T, Rouleau JR, Moyé LA, Lewis SJ, Braunwald E, AND Solomon SD. Right ventricular dysfunction and risk of heart failure and mortality after myocardial infarction. $\mathrm{J} \mathrm{Am} \mathrm{Coll} \mathrm{Cardiol}$ 39: $1450-1455,2002$. 